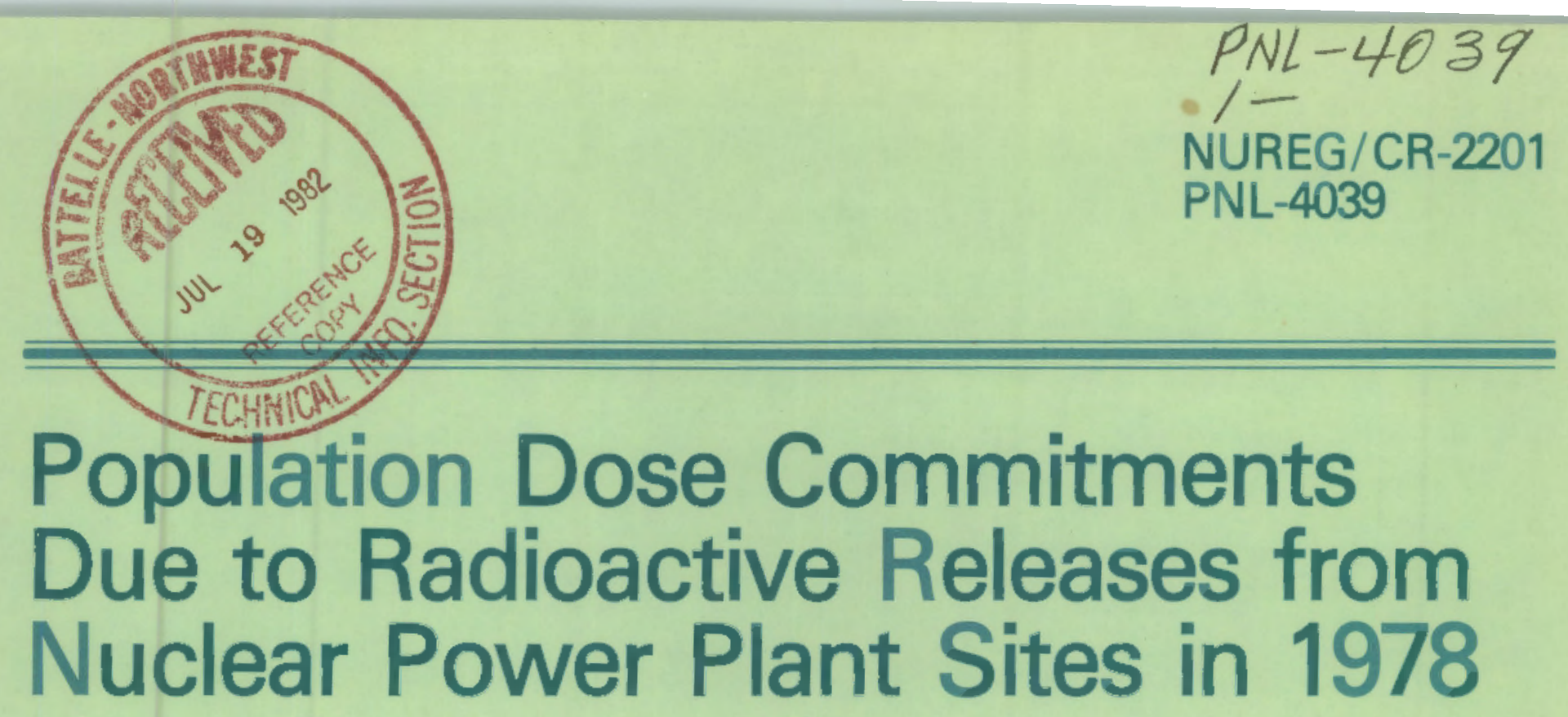

Prepared by R. A. Peloquin, J. D. Schwab, D. A. Baker

Pacific Northwest Laboratory Operated by

Battelle Memorial Institute

Prepared for

U.S. Nuclear Regulatory

Commission 


\title{
NOTICE
}

This report was prepared as an account of work sponsored by an agency of the United States Government. Neither the United States Government nor any agency thereof, or any of their employees, makes any warranty, expressed or implied, or assumes any legal liability or responsibility for any third party's use, or the results of such use, of any information, apparatus product or process disclosed in this report, or represents that its use by such third party would not infringe privately owned rights.

\author{
Available from \\ GPO Sales Program \\ Division of Technical Information and Document Control \\ U. S. Nuclear Regulatory Commission \\ Washington, D. C. 20555 \\ Printed copy price: $\$ 6.50$
}

and

National Technical Information Service Springfield, Virginia 22161 
NUREG/CR-2201

PNL-4039

\section{Population Dose Commitments Due to Radioactive Releases from Nuclear Power Plant Sites in 1978}

Manuscript Completed: April 1982

Date Published: June 1982

Prepared by

A. A. Peloquin, J. D. Schwab, D. A. Baker

Pacific Northwest Laboratory

Fichland, WA 99352

Prepared for

Division of Data Automation and Management Information

Office of Resource Management

U.S. Nuclear Regulatory Commission

Washington, D.C. 20555

NRC FIN B2243 


\section{PREVIOUS REPORTS IN THIS SERIES}

1. Population Dose Commitments Due to Radioactive Releases from Nuclear Power Plant Sites in 1975, PNL-2439, October 1977.

2. Population Dose Commitments Due to Radioactive Releases from Nuclear Power Plant Sites in 1976, NUREG/CR-1125, PNL-2940, December 1979.

3. Population Dose Commitments Due to Radioactive Releases from Nuclear Power Plant Sites in 1977, NUREG/CR-1498, PNL-3324, october 1980. 


\section{ABSTRACT}

Population radiation dose commitments have been estimated from reported radionuclide releases from commercial power reactors operating during 1978 . Fifty-year dose commitments from a one-year exposure were calculated from both liquid and atmospheric releases for four population groups (infant, child, teen-ager and adult) residing between 2 and $80 \mathrm{~km}$ from each site. This report tabulates the results of these calculations, showing the dose commitments for both liquid and airborne pathways for each age group and organ. Also included for each site is a histogram showing the fraction of the total population within 2 to $80 \mathrm{~km}$ around each site receiving various average dose commitments from the airborne pathways.

The total dose commitment from both liquid and airborne pathways ranged from a high of 200 person-rem to a low of 0.0004 person-rem with an arithmetic mean of 14 person-rem. The total population dose for all sites was estimated at 660 person-rem for the 93 million people considered at risk.

The average individual dose commitment from all pathways on a site basis ranged from a low of $3 \times 10^{-6} \mathrm{mrem}$ to a high of $0.08 \mathrm{mrem}$. No attempt was made in this study to determine the maximum dose commitment received by any one individual from the radionuclides released at any of the sites. 



\section{CONTENTS}

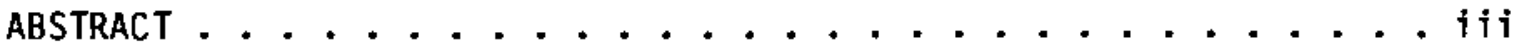
INTRODUCTION ......................... 1

Site Dependent Parameters . . . . . . . . . . . . . 2 RESULTS ......................... 7

SITE SUMMARIES.......................... 11

Arkansas .................... . . 12

Beaver Valley........................... 14

Big Rock Point . . . . . . . . . . . . . . . 16

Browns Ferry 1, 2, \&3 . . . . . . . . . . . 18

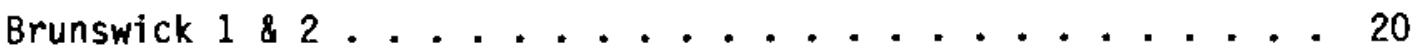

Calvert Cliffs $1 \& 2$. . . . . . . . . . . . . 22

Cook $1 \& 2$ \&..................... 24

Cooper Station .................... 26

Crystal River.................. . . 28

Davis-Besse . . . . . . . . . . . . . . 30

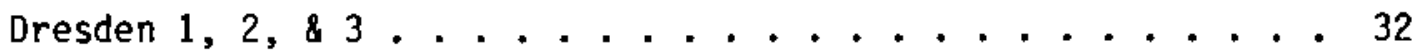

Duane Arnold ................... . . . 34

J. M. Farley ................... 36

J. A. Fitzpatrick . . . . . . . . . . . . . 38

Fort Calhoun ..................... 4 40

R. E. Ginna ...................... 4 42

Haddam Neck (Connecticut Yankee) . . . . . . . . . . . 44 
Edwin I. Hatch ................... 46

Humboldt Bay ................. 48

Indian Point $1,2 \& 3 \ldots \ldots \ldots$

Kewaunee .................... 52

LaCrosse ................... . . 54

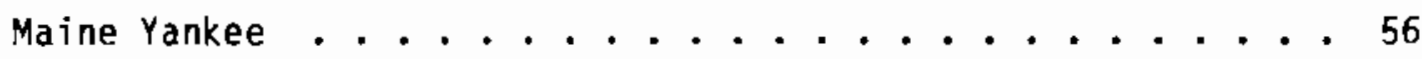

Millstone Point $1 \& 2 \ldots \ldots \ldots$

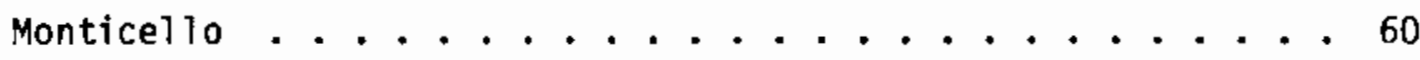

Nine Mile Point......................... 62

North Anna .................... 64

Oconee $1,2 \& 3 \ldots \ldots \ldots 6$

oyster creek ..................... 68

Palisades ....................... 70

Peach Bottom $2 \& 3 \ldots \ldots \ldots \ldots \ldots$

Pilgrim ........................ 74

Point Beach $1 \& 2 \ldots \ldots \ldots 76$

Prairie Island $1 \& 2 \ldots \ldots \ldots 78$

Quad Cities $1 \& 2 \ldots \ldots . \ldots . \ldots 80$

Rancho Seco.................... 82

H. B. Robinson .................. 84

St. Lucie..................

Salem .............................. 88

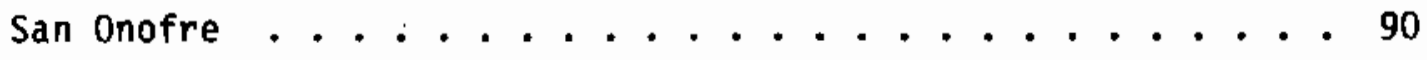

Surry $1 \& 2 \ldots \ldots 92$ 
Three Mile Island $1 \& 2$. . . . . . . . . . . . 94

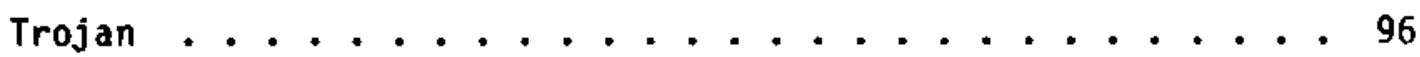

Turkey Point $3 \& 4$. . . . . . . . . . . . . . 98

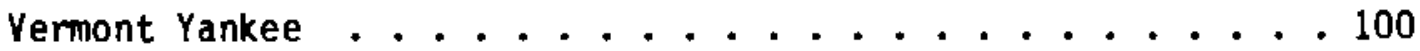

Yankee Rowe .. . . . . . . . . . . . . . 102

Zion 182 . . . . . . . . . . . . . . 104

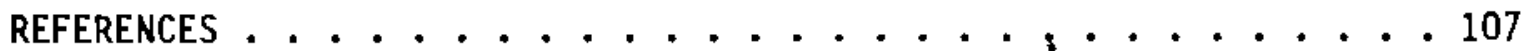

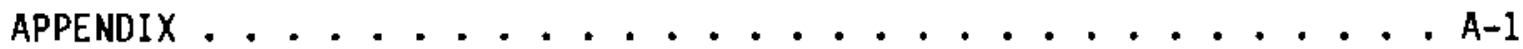


The Pacific Northwest Laboratory (PNL) is operated by Battelle Memorial Institute for the Department of Energy (DOE). This study, sponsored by the Nuclear Regulatory Commission (NRC) and conducted at PNL, estimates radiation dose commitments from reported radionuclide releases at 67 commercial power reactors operating during 1978. This work is a continuation of the study done for 1975, 1976 and 1977 releases (Baker, Soldat and Watson 1977, Baker 1979 and 1980). In this study, as in the last, we estimated the population (collective) dose commitment (a) from both the liquid and gaseous releases to four age groups making up the population residing in the region of the site: infant ( 0 to $1 \mathrm{yr}$ ), child (1 to $11 \mathrm{yr}$ ), teen-ager (11 to $17 \mathrm{yr}$ ) and adult (17 $\mathrm{yr}$ and older).

The particular organs of reference in this study are listed in Table 1. The major pathways by which radionuclides travel from the reactor to the individual receptors are shown in Table 2. Other possible liquid pathways such as direct exposure from waterborne activities (swinming, boating, shoreline recreation) and internal exposure through ingestion of food produced using contaminated irrigation water were not included. This was because we have found from past experience that the doses from these pathways is generally much smaller than the doses from the pathways considered in this study.

The regional population for which we estimated doses included those persons estimated to be living in a region between 2 and $80 \mathrm{~km}$ around the reactor sites during 1978. We estimated the numbers of persons by extrapolating Bureau of Census data for 1970 to 1978 . Atmospheric transport factors (annual average dilution and annual average deposition) were calculated for the region around each site using appropriate meteorological data supplied by the NRC's Office of Nuclear Reactor Regulation. To calculate the doses, we used models approved by the NRC. We incorporated these models into two small computer codes to expedite the dose calculations involved for each site.

Site-specific parameters other than releases, meteorology and population were obtained from environmental statements for the various reactors when available (Table 3 ). Such parameter values include the total population drinking contaminated water, river flow, dilution flow from the reactors (for sites not on rivers), fish and invertebrate harvest for region, and dilution factors for drinking water and aquatic foods.

(a) As used in this report, dose commitment describes the total-body dose equivalent received over 50 years from intake during the year 1978. 
The reactors included in this study, their type, licensed thermal power rating and net electrical output for 1978 are listed in Table 4 . Those reactors which had an operating augmented gaseous radioactive waste system in 1978 are identified in the table. Populations at risk and the dose conmitments derived in the study are also tabulated.

Site-Dependent Parameters

The section entitled Site Summaries gives the location (including latitude and longitude) for each reactor stte and the estimated 1978 population within 2 to $80 \mathrm{~km}$ around the site. This population is derived from the 1970 census by extrapolation to 1978. In addition, the location of major metropolitan centers within $80 \mathrm{~km}$ are listed along with their 1978 extrapolated populations. The populations of the Standard Metropolitan Statistical Areas (SMSA) are given where applicable. Next, the site-specific data pertinent to the airborne pathways are specified. The average production rates of vegetable crops and animal products are given for the area within an $80-k m$ radius based upon the statewide average. This production has been reduced for sites on lakes and seacoasts to account for the presence of the body of water. An animal grazing factor is estimated for each site location. This factor accounts for the fraction of the year during which grazing animals such as milk cows and beef cattle graze on fresh pasture in the region around the site. After average production rates are given, the period of record and the percent data recovery of the meteorological data used in calculating diffusion factors is indicated.

Various site-dependent factors associated with the waterborne pathways are presented next. For lake and ocean sites, we used the average dilution of plant effluents for the year 1978 specified by Tichler and Benkovitz (1981). For river sites, the average annual river flow is tabulated. This flow was used in place of a dilution flow from the plant to account for dilutions of liquid releases at the locations of probable intake of drinking water and aquatic food catch. Any exceptions to this scheme have been footnoted. Next is shown the estimated 1978 population utilizing drinking water drawn from supplies containing diluted effluents from the site. These are shown with an estimated dilution factor where applicable. Fish and invertebrate catch data taken from the respective plant environmental statement, when available (see Table 3), are listed next, along with estimated dilution factors for the lake and ocean sites. 
TABLE 1. Organs Considered in This Study

\begin{tabular}{|c|c|}
\hline $\begin{array}{l}\text { Organs Affected by } \\
\text { Airborne Releases }\end{array}$ & $\begin{array}{l}\text { Organs Affected by } \\
\text { Waterborne Releases }\end{array}$ \\
\hline $\begin{array}{l}\text { Total body } \\
\text { Thyroid } \\
\text { Bone } \\
\text { GI tract } \\
\text { Liver }\end{array}$ & $\begin{array}{l}\text { Total body } \\
\text { Thyroid } \\
\text { Bone } \\
\text { GI tract } \\
\text { Liver }\end{array}$ \\
\hline
\end{tabular}

TABLE 2. Pathways Considered in This Study by which Radionuclides Travel from Reactors to Persons

Pathways for

Airborne Releases

Air submersion

Contaminated ground

Inhalation

Ingestion of food crops

and animal products
Pathways for Waterborne Releases

Ingestion of drinking water Ingestion of fish and invertebrates 


\section{TABLE 3. Environmental Statements for Power Plants Included in this Study}

\begin{tabular}{|c|c|c|c|c|}
\hline $\begin{array}{l}\text { Site } \\
\text { Number }\end{array}$ & Reactor Site & $\begin{array}{l}\text { Docket } \\
\text { Number }\end{array}$ & Date & Remarks \\
\hline 1 & Big Rock Point & & & ES(a) not available \\
\hline 2 & Brown's Ferry $1,2,3$ & & Jul 71 & $\begin{array}{l}\text { ES Published by Tenn. } \\
\text { Valley Authority }\end{array}$ \\
\hline 3 & Cooper Station & $50-298$ & Feb 73 & Draft ES \\
\hline 4 & Bresden & & & ES of Dresten 2,3 used \\
\hline 4 & Dresden 2,3 & $50-237,50-249$ & Noy 73 & \\
\hline 5 & Beaver Valley & $50-334$ & Ju1 73 & \\
\hline 6 & Humboldt Bay & & & ES not available \\
\hline 7 & Lacrosse & $50-409$ & Jun 76 & Draft ES \\
\hline 8 & Milistone point 1,2 & $50-245,50-336$ & Jun 73 & \\
\hline 9 & Monticello & $50-263$ & Nov 72 & \\
\hline 10 & Nine sile Point & $50-220$ & $\operatorname{Jan} 74$ & \\
\hline 11 & Oyster Creek & $50-219$ & Dec 74 & \\
\hline 12 & Peach Rottom 2,3 & $50-277,50-278$ & Apr 73 & \\
\hline 13 & Pilgrim & $50-293$ & : May 72 & \\
\hline 14 & Quad Cities 1,? & $50-254,50-265$ & $\operatorname{sep} 72$ & \\
\hline 15 & Vermont Yankee & $50-271$ & Ju1 72 & \\
\hline 16 & St. Lucie & $50-335$ & Jun 73 & \\
\hline 17 & Brunswick 1,2 & $50-324,50-325$ & Jun 73 & Draft IS \\
\hline 18 & Duane Arnold & $50-331$ & Mar 73 & \\
\hline 19 & J. A. Fitzpatrick & $50-333$ & Mar 73 & \\
\hline 20 & E. I. Hatch & $50-321$ & oct 72 & \\
\hline 21 & arkansas & $50-313$ & Feb 73 & \\
\hline 22 & $\begin{array}{l}\text { Connecticut Yankee } \\
\text { (Haddam lieck) }\end{array}$ & $50-213$ & oct 73 & \\
\hline 23 & Fort Calhoun & $50-285$ & Aug 72 & \\
\hline 24 & H. B. Robinson & $50-261$ & Apr 74 & \\
\hline 25 & Indian Point 1,2 & $50-247$ & Sep 72 & ES of Indian Point 2 used \\
\hline 26 & Salen & $50-272,50-311$ & Apr 73 & \\
\hline 27 & Kewaunee & $50-305$ & $\operatorname{Sec} 72$ & \\
\hline 28 & Maine Yankee & $50-309$ & Jul 72 & \\
\hline 29 & Oconee $1,2,3$ & $50-269,50-270$ & Mar 72 & \\
\hline & & $50-287$ & & \\
\hline 30 & Palisades & $50-255$ & dun 72 & \\
\hline 31 & Point Beach 1,2 & $50-266,50-301$ & Yay 72 & \\
\hline 32 & Prairie Island 1,2 & $50-282,50-306$ & May 73 & \\
\hline 33 & R. E. Ginna & $50-244$ & Dec 73 & \\
\hline 34 & San Onofre & $50-206$ & oct 73 & \\
\hline 35 & Surry 1,2 & $50-281$ & Jun 72 & ES of Surry 2 used \\
\hline 36 & Three Mile Island & $50-289$ & Dec 72 & \\
\hline $\begin{array}{l}37 \\
38\end{array}$ & $\begin{array}{l}\text { Turkey Point 3,4 } \\
\text { Yankee Rowe }\end{array}$ & $50-250,50-251$ & Feb 72 & $\begin{array}{l}\text { Draft ES } \\
\text { ES not availahle }\end{array}$ \\
\hline 39 & Zion 1,2 & $50-295,50-304$ & Dec 72 & \\
\hline 40 & Calvert Cliffs & $50 \cdot 317$ & Apr 73 & \\
\hline 41 & Cook & $50-315$ & Aug 73 & \\
\hline 92 & Trojan & $50-344$ & Jan 73 & Draft ES \\
\hline 43 & Rancho Seco & $50-312$ & Mar 73 & \\
\hline 44 & Crystal River & $50-302$ & May 73 & \\
\hline 45 & Davis-Besse & $50-346$ & Mar 73 & \\
\hline 46 & J. M. Farley(b) & $50-348,50-369$ & Jun 72 & \\
\hline 47 & North Anna(b) & $50-338,50-339$ & Apr 73 & \\
\hline
\end{tabular}

(a) Environmental Statement

(b) Added for 1978 
TABLE 4. Reactor Characteristics and Population Total-Body Dose Commitments, 1978

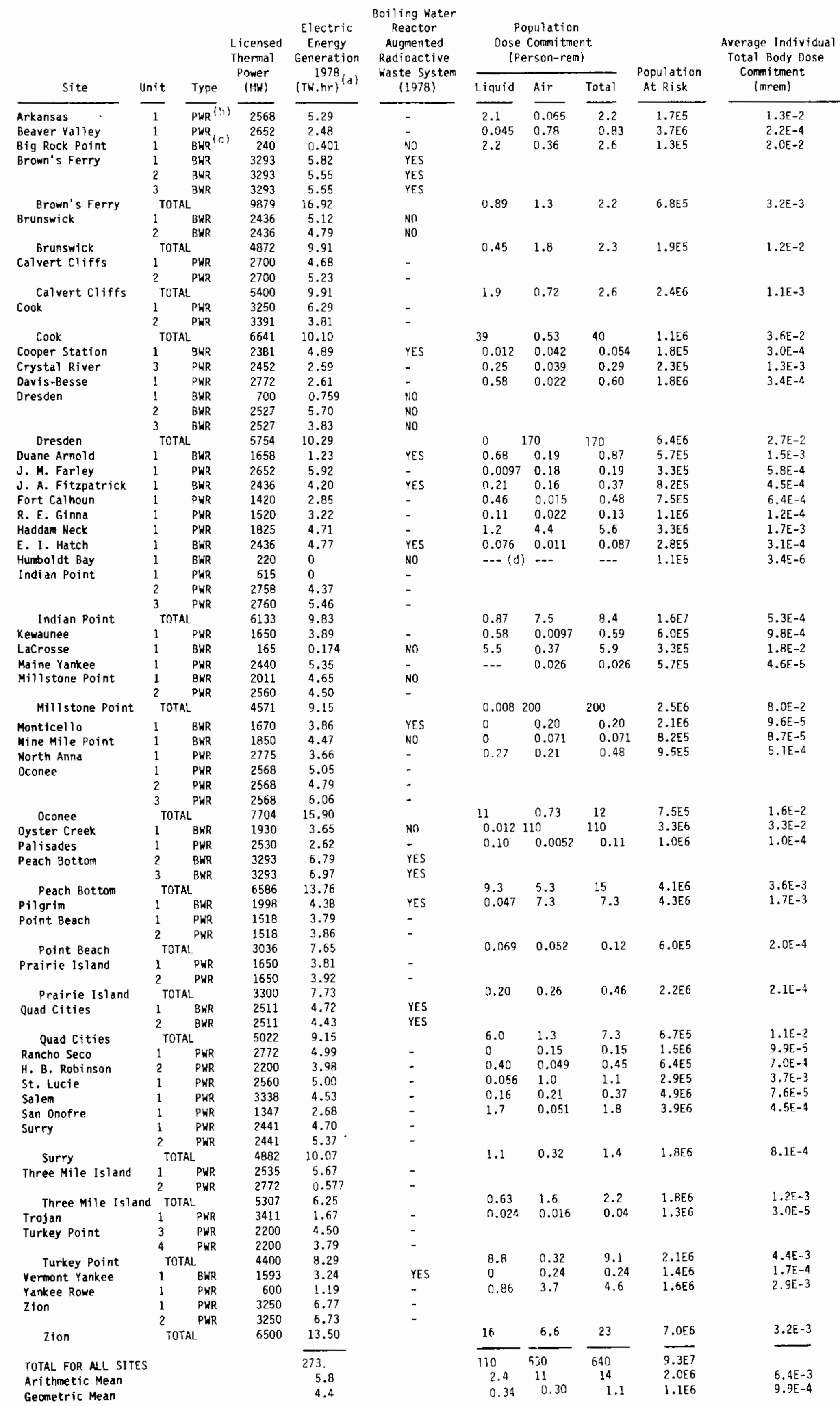

(a) 1 TW.hr $=3.6 E 15$ joules

(c) Boiling water reactor

(d) Indicates dose commitment $<0.001$ person-rem. 

This report consists of a summary of values used for site-specific parameters at each site, as explained above, and the results of population dose commitment calculations. The population dose commitments are presented in two tables facing the page summarizing site-specific parameters for that site. These tabies include both liquid and airborne pathway dose commitments for the several organs of reference for each age group investigated. They al so include the dose to the whole population which includes all age groups. The airborne population dose commitments for each of 160 segments partitioning the region around the site(a) were divided by the population residing within that segment to derive an average individual dose for that segment. These doses are summarized as a histogram showing percent of the population receiving a given dose level for each site. The fractional population dose from the liquid pathway was not determined in this manner, because the NRC does not at present take into account the location of individuals exposed via this pathway, except those exposed through ingestion of drinking water.

Population dose commitments estimated for both the liquid pathways and airborne pathways varied widely over the 47 sites (67 reactors) studied. The total dose commitments (from both pathways) varied from a high of 200 to a low of 0.0004 person-rem. The arithmetic mean for the dose from liquid pathways was 3 person-rem and the mean for the dose from airborne pathways was 11 person-rem (see Table 4 ).

Releases from Monticello, Nine Mile Point, Rancho Seco, Vermont Yankee and Dresden resulted in the smallest doses from liquid pathways (zero to near zero, respectively). This was because no liquid releases were reported for Nine Mile Point, Vermont Yankee, Monticello and Rancho Seco, and the receiving waters for the Dresden site are contaminated with Chicago sewage to such an extent as to severeiy limit use of this water for drinking or fishing. The largest liquid pathway doses to the total body calculated were 39 person-rem at cook. These doses resulted primarily from the radioactive cesium (Cs-134 and Cs-137) released by these plants.

The lowest total airborne pathway dose to the total body was estimated for Humboldt Bay $(0.0002$ person-rem); while the highest doses were at Millstone Point (200 person-rem) and Dresden (170 person-rem). The major contributors

(a) See Appendix for definition of segments. 
to these doses were the noble gases: $\mathrm{Kr}-88$ and $\mathrm{Xe}-135$. The total population dose commitments from all sites for 1978 were estimated to be 130 person-rem via liquid pathways and 530 person-rem via the airborne pathways (Table 4 ). Compared to 1977, the "liquid dose" is 1ower (130 vs. 160); and al so, the "air dose" is lower (530 vs. 540). Table 5 compares the total population dose commitments estimated for the past four years.

We should point out here, however, that the doses estimated in this study are extremely low compared to an average annual background dose of 0.1 rem, and they are well within all limits. Even the highest site average individual dose commitment of 0.08 mrem (Table 4) from one site (Milestone Point) is well within any national limits.

Figure 1 shows graphically the wide range of the airborne population dose commitments for the reactor sites. The median, upper and lower quartiles and upper and lower octiles for the distribution of doses calculated for each of 160 segments are indicated for each site. Figure 2 is a histogram for all 47 sites taken together. We can see from this plot that about $24 \%$ of the total population at risk (93 million) would each receive a dose commitment of between 0.0003 and 0.001 mrem. We can see further that $<0.6 \%$ receive a dose which is less than $1 \times 10^{-6}$ mrem. Although not discernible from the plot, $0.01 \%$ received a dose of between 3 and $10 \mathrm{mrem}$. There were no average dose commitments greater than $10 \mathrm{mrem}$. However, no attempt was made in this study to estimate the maximum dose commitment received by any one individual from the radionuclides released at any of the sites.

It should be noted that we have been comparing dose commitments calculated in this study with annual background. This comparison is not quite exact, since these dose commitments are those total-body doses received from the year's (1978) effluent release, over 50 years of a person's lifetime. However, most of the dose commitment calculated here is delivered in the first year, so the comparison is reasonably valid.

TABLE 5. Comparison of Annual Population Dose Commitments for the Last Four Years (person-rem)

\begin{tabular}{lrrrr} 
& \multicolumn{1975}{c}{} & $\underline{1976}$ & $\underline{1977}$ & $\underline{1978}$ \\
Liquid & 76 & 82 & 160 & 130 \\
Air & $\underline{1300}$ & $\underline{390}$ & $\underline{540}$ & $\underline{530}$ \\
TOTAL & 1400 & 470 & 700 & 660
\end{tabular}




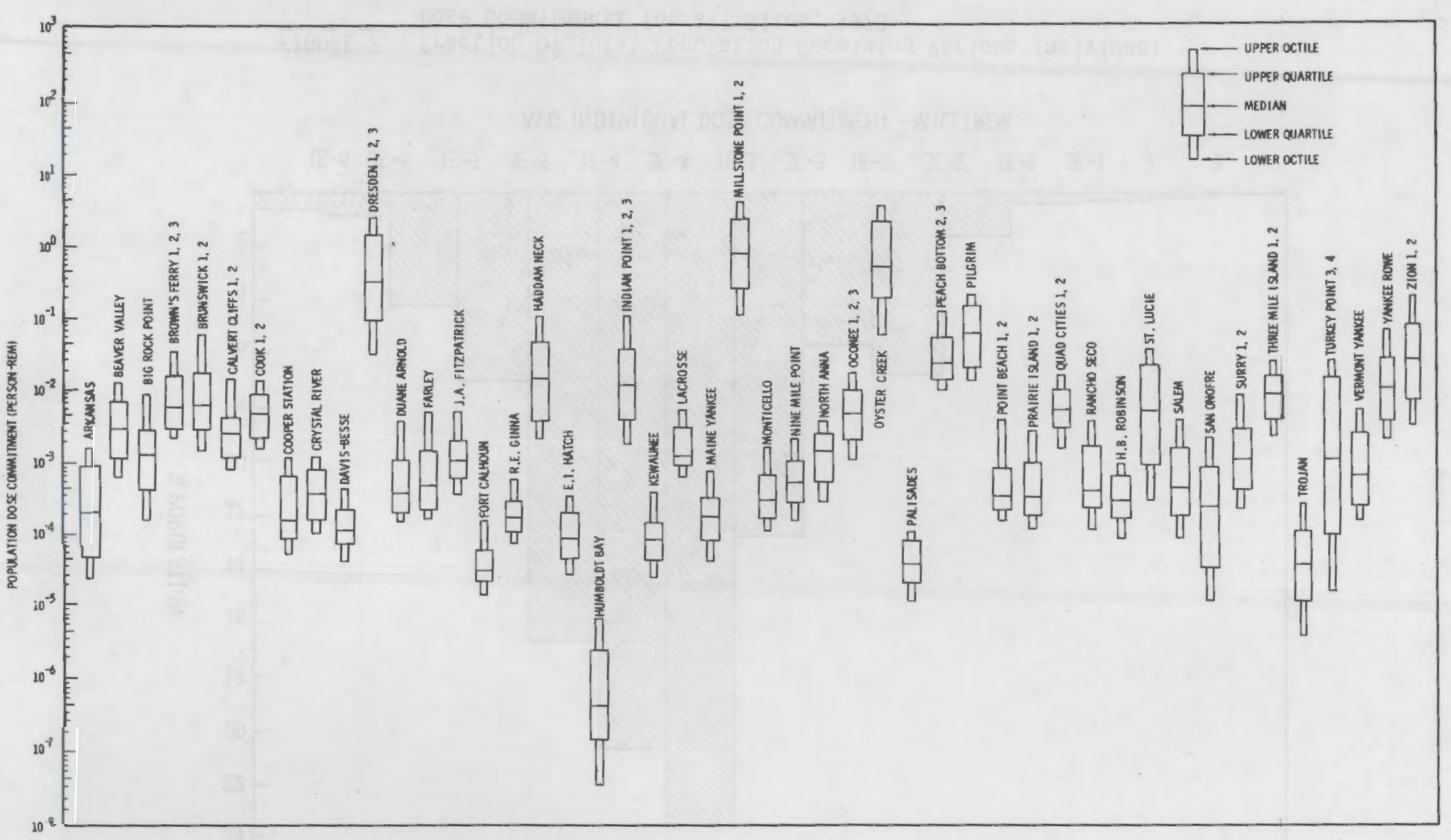

FIGURE 1. Airborne Population Dose Commitments for the Reactor Sites, 1978 


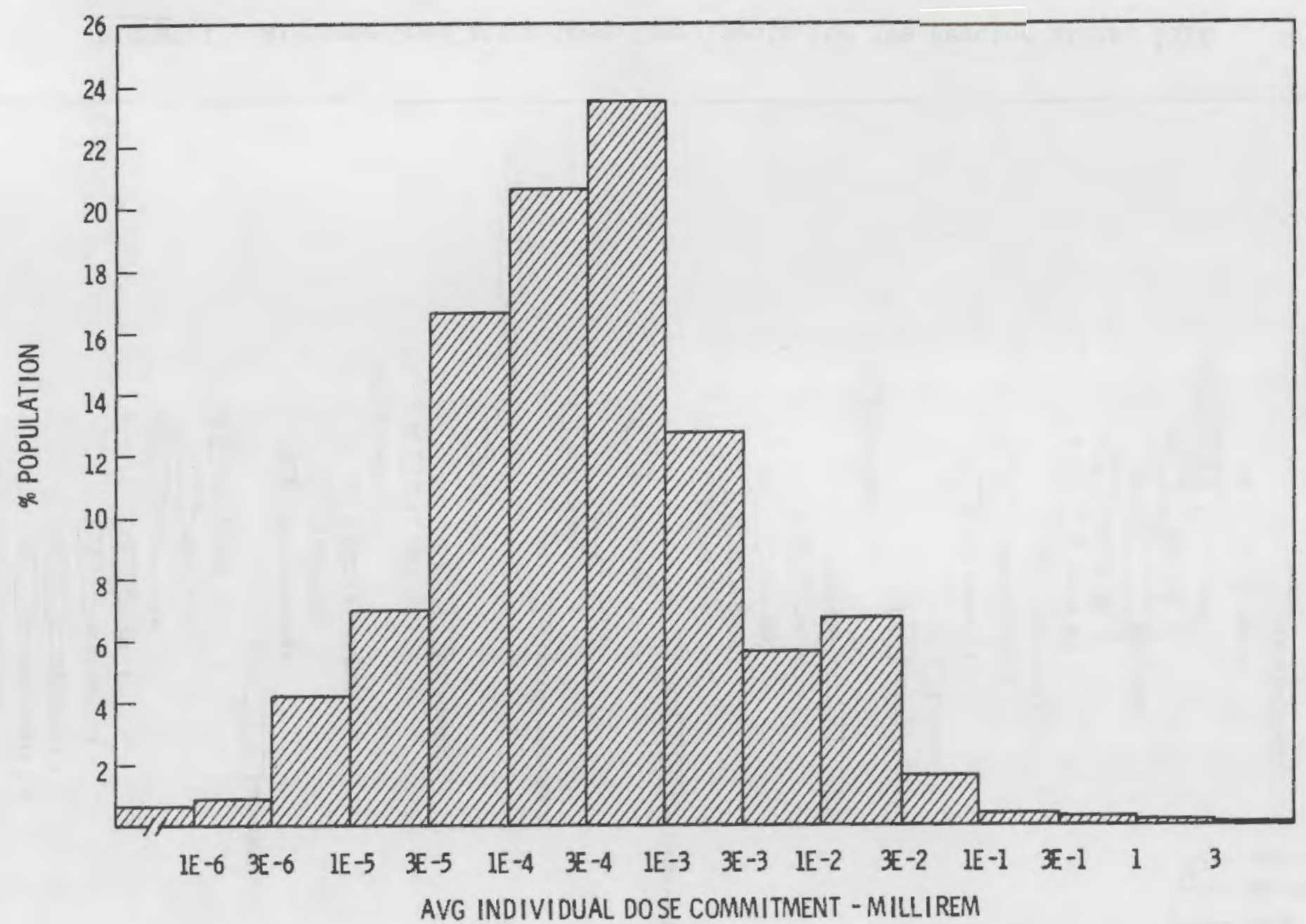

FIGURE 2. Fraction of Total Population Receiving Various Individual Dose Commitments for all Sites, 1978 


\section{SITE SUMMARIES}

\section{8}


Location: $\quad$ N $35.30750 \quad$ W 93.23080

POPULATION DATA

Total Population Within 20-to-80-km Region: $1.7 E 5$

\begin{tabular}{|c|c|c|}
\hline Center & Population & Location \\
\hline $\begin{array}{l}\text { Russellville } \\
\text { Conway }\end{array}$ & $\begin{array}{l}13,000 \\
18,000\end{array}$ & $10 \mathrm{~km}$ \\
\hline
\end{tabular}

SITE SPECIFIC DATA - AIRBORNE PATHWAYS

Average Annual State Production

of Crops and Animal Products

In $80-\mathrm{km}$ Radius Circle

Regional Productivity Factor:

Animal Grazing Factor:
Veg: $5.8 \mathrm{E} 6 \mathrm{kilogram}$

Milk: 4.8E7 liter

Meat: $7.2 E 7$ kilogram

1

0.7

Meteorology Period of Record: 1 JAN 75 - 31 DEC 75 Recovery: 97\%

SITE SPECIFIC DATA - WATERBORNE PATHWAYS

Average Arkansas River

Flow at site: $36,000 \mathrm{ft}^{3} / \mathrm{s}$

Drinking Water:

Exposed Population: None

Fish:

Edible Harvest: $1.4(\mathrm{a}) \mathrm{kg} / \mathrm{yr}$

Dilution Factor: 1

(a) Average individual consumption rates as given in the FES (1973) were used in lieu of catch data. 
AVERAGE INDIVIDUAL DOSE-COMMITMENT HISTOGRAM FOR

ARKANSAS

Dose Commitments (person-rem) from Liquid Pathways

Total Body GI-LLI Thyroid Bone Liver

$\begin{array}{llllll}\text { Infant } & 0.0 E+00 & 0.0 E+00 & 0.0 E+00 & 0.0 E+00 & 0.0 E+00 \\ \text { Child } & 8.9 E-02 & 1.3 E-02 & 1.3 E-02 & 4.3 E-01 & 5.0 E-01 \\ \text { Teen } & 1.7 E-01 & 2.7 E-02 & 9.7 E-03 & 2.6 E-01 & 4.3 E-01 \\ \text { Adult } & 1.9 E+00 & 2.3 E-01 & 6.4 E-02 & 1.5 E+00 & 2.6 E+00 \\ \text { TOTAL } & 2.1 E+00 & 2.7 E-01 & 8.7 E-02 & 2.2 E+00 & 3.5 E+00\end{array}$

Dose Commitments (person-rem) from Airborne Pathways

Total Body GI-LLI Thyroid Bone Liver Lung

$\begin{array}{lllllll}\text { Infant } & 9.4 \mathrm{E}-04 & 9.3 \mathrm{E}-04 & 1.4 \mathrm{E}-03 & 9.5 \mathrm{E}-04 & 9.6 \mathrm{E}-04 & 1.0 \mathrm{E}-03 \\ \text { Child } & 1.0 \mathrm{E}-02 & 1.0 \mathrm{E}-02 & 1.3 \mathrm{E}-02 & 1.0 \mathrm{E}-02 & 1.1 \mathrm{E}-02 & 1.1 \mathrm{E}-02 \\ \text { Teen } & 7.6 \mathrm{E}-03 & 7.6 \mathrm{E}-03 & 8.7 \mathrm{E}-03 & 7.6 \mathrm{E}-03 & 7.7 \mathrm{E}-03 & 8.8 \mathrm{E}-03 \\ \text { Adult } & 4.6 \mathrm{E}-02 & 4.6 \mathrm{E}-02 & 5.0 \mathrm{E}-02 & 4.6 \mathrm{E}-02 & 4.6 \mathrm{E}-02 & 5.0 \mathrm{E}-02 \\ \text { TOTAL } & 6.5 \mathrm{E}-02 & 6.5 \mathrm{E}-02 & 7.3 \mathrm{E}-02 & 6.5 \mathrm{E}-02 & 6.5 \mathrm{E}-02 & 7.1 \mathrm{E}-02\end{array}$

Production/Consumption factors: (a)

Produce: <1 Milk: 2.2 Meat: 5.4

FRACTION OF POPULATION RECEIVING AN INDICATED AVERAGE TOTAL-80DY DOSE COMMITMENT FROM AIRBORNE PATHWAYS

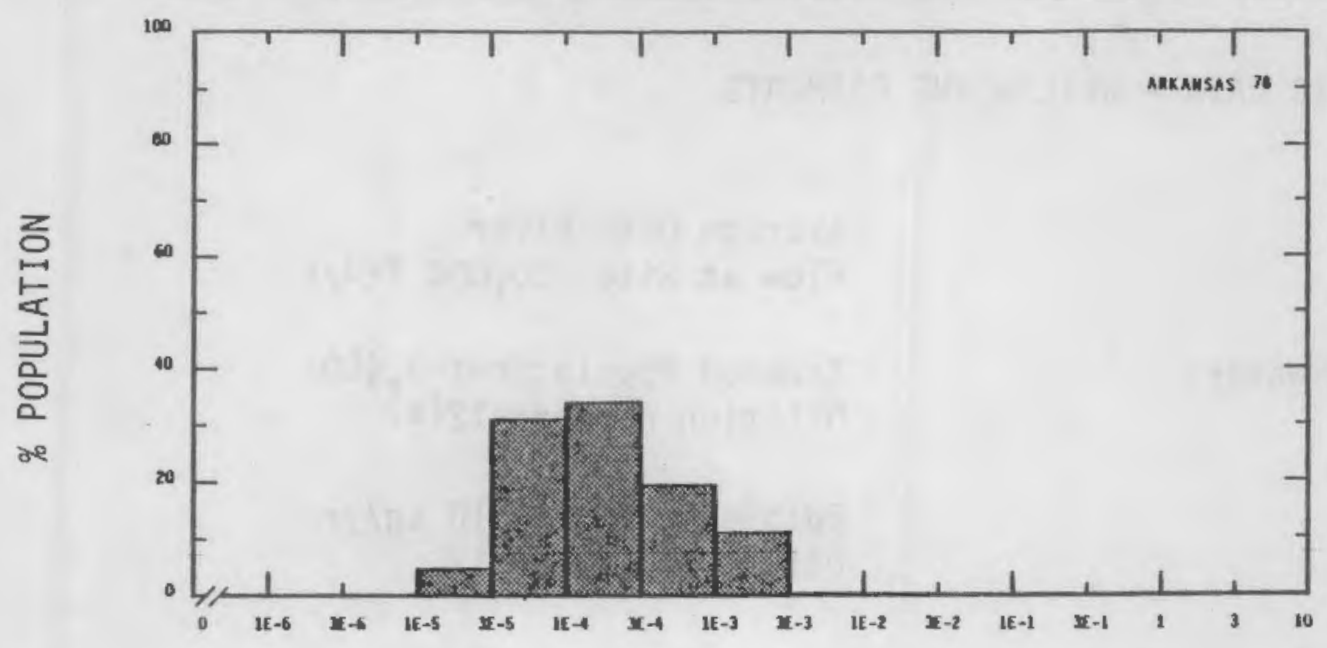

AVG INDIVIDUAL DOSE COMMITMENT - MILLIREM

(a) See Appendix A, Page A-5, for explanation of this ratio. 
Location: $\quad$ N $40.6214^{\circ} \quad$ W 80.43470

Total Population Within 20-to-80-km Region: $3.7 E 6$

Major Metropolitan Centers Within Region:

\begin{tabular}{|c|c|c|c|}
\hline Center & Population & \multicolumn{2}{|c|}{ Location } \\
\hline Pittsburgh SMSA & $2.41 E 6$ & $32 \mathrm{~km}$ & SE \\
\hline Youngstown-Warren SMSA & 530,000 & $56 \mathrm{~km}$ & NNW \\
\hline Stuebenville & 31,000 & $31 \mathrm{~km}$ & SW \\
\hline Wheeling & 48,000 & $69 \mathrm{~km}$ & SSW \\
\hline
\end{tabular}

SITE SPECIFIC DATA - AIRBORNE PATHWAYS

Average Annual State Production of Crops and Animal Products

In $80-\mathrm{km}$ Radius Circle

Regional Productivity Factor:

Animal Grazing Factor:
Yeg: $5.3 E 7$ kilogram

Mi1k: 5.3E8 liter

Meat: $5.4 \mathrm{E} 7 \mathrm{kilogram}$

1

0.5

Meteorology Period of Record: 1 JAN 77 - 31 DEC 77 Recovery: 92\%

SITE SPECIFIC DATA - WATERBORNE PATHWAYS

Average Ohio River

Flow at Site: $30,000 \mathrm{ft}^{3} / \mathrm{s}$

Drinking Water:

Exposed Population: 6,400

Dilution Factor: 12 (a)

Fish:

Edible Harvest: $410 \mathrm{~kg} / \mathrm{yr}$

Dilution Factor: 1

(a) This factor accounts for the incomplete dilution of plant effluent by river at point of drinking water intake at Midland. 
POPULATION DOSE-COMMITMENT ESTIMATES AND

AVERAGE INDIVIDUAL DOSE-COMMITMENT HISTOGRAM FOR

BEAYER YALLEY

Dose Commitments (person-rem) from Liquid Pathways

Total Body GI-LLI Thyroid Bone Liver

$\begin{array}{llllll}\text { Infant } & 7.9 \mathrm{E}-04 & 7.7 \mathrm{E}-04 & 2.5 \mathrm{E}-03 & 1.7 \mathrm{E}-04 & 9.9 \mathrm{E}-04 \\ \text { Child } & 9.0 \mathrm{E}-03 & 8.8 \mathrm{E}-03 & 2.0 \mathrm{E}-02 & 1.9 \mathrm{E}-03 & 1.1 \mathrm{E}-02 \\ \text { Teen } & 3.6 \mathrm{E}-03 & 3.6 \mathrm{E}-03 & 6.9 \mathrm{E}-03 & 4.9 \mathrm{E}-04 & 4.0 \mathrm{E}-03 \\ \text { Adult } & 3.1 \mathrm{E}-02 & 3.1 \mathrm{E}-02 & 5.3 \mathrm{E}-02 & 3.0 \mathrm{E}-03 & 3.2 \mathrm{E}-02 \\ \text { TOTAL } & 4.5 \mathrm{E}-02 & 4.4 \mathrm{E}-02 & 8.3 \mathrm{E}-02 & 5.6 \mathrm{E}-03 & 4.8 \mathrm{E}-02\end{array}$

Dose Commitments (person-rem) from Airborne Pathways

Total Body. GI-LLI Thyroid Bone Liver Lung

$\begin{array}{lllllll}\text { Infant } & 1.3 \mathrm{E}-02 & 1.3 \mathrm{E}-02 & 4.8 \mathrm{E}-02 & 1.7 \mathrm{E}-03 & 1.3 \mathrm{E}-02 & 1.3 \mathrm{E}-02 \\ \text { Child } & 1.6 \mathrm{E}-01 & 1.6 \mathrm{E}-01 & 3.5 \mathrm{E}-01 & 1.9 \mathrm{E}-02 & 1.6 \mathrm{E}-01 & 1.6 \mathrm{E}-01 \\ \text { Teen } & 9.8 \mathrm{E}-02 & 9.8 \mathrm{E}-02 & 1.8 \mathrm{E}-01 & 1.3 \mathrm{E}-02 & 9.8 \mathrm{E}-02 & 1.0 \mathrm{E}-01 \\ \text { Adult } & 5.2 \mathrm{E}-01 & 5.2 \mathrm{E}-01 & 8.0 \mathrm{E}-01 & 8.0 \mathrm{E}-02 & 5.2 \mathrm{E}-01 & 5.3 \mathrm{E}-01 \\ \text { TOTAL } & 7.8 \mathrm{E}-01 & 7.8 \mathrm{E}-01 & 1.4 \mathrm{E}+00 & 1.1 \mathrm{E}-01 & 7.8 \mathrm{E}-01 & 8.0 \mathrm{E}-01\end{array}$

Production/Consumption factors:

Produce: <1 Milk: 1.1 Meat: <1

FRACTION OF POPULATION RECEIVING AN INDICATED AVERAGE TOTAL-BODY DOSE COMMITMENT FROM AIRBORNE PATHWAYS

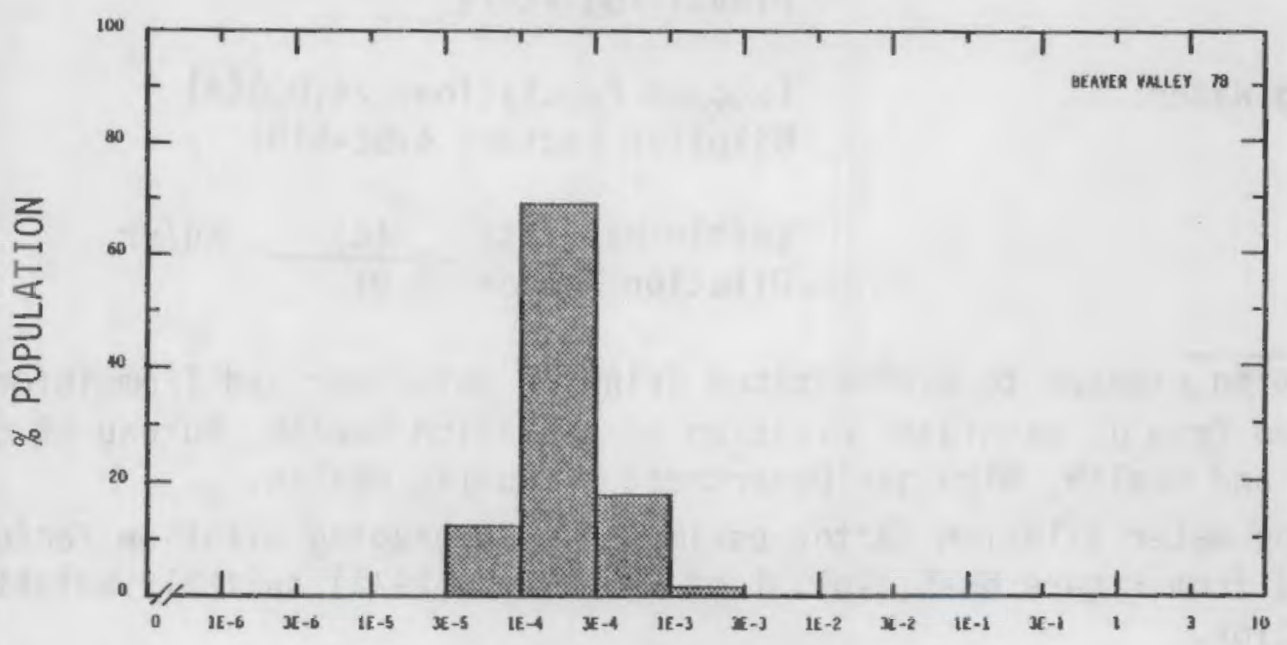

AVG INDIVIOUAL DOSE COMMITMENT - MILLIREM 
Location: N $45.35890 \quad$ W 85.19580

POPULATION DATA

Total Population Within 20-to-80-km Region: 1.3E5

Major Metropolitan Centers Within Region:

\begin{tabular}{ccc} 
Center & Population & \multicolumn{2}{c}{ Location } \\
\cline { 2 - 3 } & 19,000 & $70 \mathrm{~km} \quad$ SSW
\end{tabular}

SITE SPECIFIC DATA - AIRBORNE PATHWAYS

Average Annual State Production

of Crops and Animal Products

In $80-\mathrm{km}$ Radius Circle

Regional Productivity Factor:

Animal Grazing Factor:
Veg: $6.9 \mathrm{E} 7 \mathrm{kilogram}$

Milk: 2.9E8 liter

Meat: $4.5 \mathrm{E} 7 \mathrm{kilogram}$

0.5

0.5

Meteorology Period of Record: 9 FEB 61 - 8 FEB 63 Recovery: 85\%

SITE SPECIFIC DATA - WATERBORNE PATHWAYS

Average Dilution Flow from

Plant: $108 \mathrm{ft}^{3} / \mathrm{s}$

Drinking Water:

Exposed Population: 24,000(a)

Dilution Factor: $4.9 \mathrm{E}-5(\mathrm{~b})$

Fish:

Edible Harvest: $\quad$ (c) $\mathrm{kg} / \mathrm{yr}$

Dilution Factor: 0.01

(a) Population exposed to contaminated drinking water derived from information obtained from J. Hennigan, Division of Radiation Health, Bureau of Environmental and Health, Michigan Department of Public Health.

(b) Drinking water dilution factor estimated by averaging dilution factor derived from Figure 6B-5, Vol. 1 of WASH-1258 (1973) suitably weighted for population.

(c) Generic consumption rate used (Table A-1). 
POPULATION DOSE-COMMITMENT ESTIMATES AND

AVERAGE INDIVIDUAL DOSE-COMMITMENT HISTOGRAM FOR

BIG ROCK POINT

Dose Commitments (person-rem) from Liquid Pathways

Total Body GI-LLI Ihyroid Bone Live

Infant

3.7E-07

$7.1 \mathrm{E}-08$

$3.7 \mathrm{E}-08$

3.1E-06

3.5E-06

Child

$8.9 \mathrm{E}-02$

$3.8 \mathrm{E}-03$

3.9E-06

5. $6 \mathrm{E}-01$

5.7E-01

Teen

1.7E-01

7.2E-03

3. $3 \mathrm{E}-01$

3. $3 E-01$

4.7E-01

Adult

1. $9 E+00$

5. $9 \mathrm{E}-02$

2.7E-05

1. $9 E+00$

$2.8 \mathrm{E}+00$

TOTAL

2. $2 E+00$

7. $0 \mathrm{E}-02$

3. $4 \mathrm{E}-05$

2. $8 E+00$

3. $8 \mathrm{E}+00$

Dose Commitments (person-rem) from Airborne Pathways

Total Body GI-LLI Thyroid Bone Liver Lung

Infant

$5.1 E-03$

$5.1 \mathrm{E}-03$

$5.3 \mathrm{E}-03$

$5.1 \mathrm{E}-03$

$5.1 \mathrm{E}-03$

$5.3 \mathrm{E}-03$

Child

$5.7 \mathrm{E}-02$

$5.7 \mathrm{E}-02$

5.9E-02

$5.7 \mathrm{E}-02$

5.7E-02

$5.9 \mathrm{E}-02$

Teen

4.2E-02

4.2E-02

4.3E-02

4.2E-02

4.2E-02

4. $4 \mathrm{E}-02$

Adult

2. $5 \mathrm{E}-01$

2.5E-01

2. $6 \mathrm{E}-01$

2.5E-01

2.5E-01

2.6E-01

TOTAL

3. $6 \mathrm{E}-01$

3. $6 \mathrm{E}-01$

3.6E-01

3. $6 \mathrm{E}-01$

3.6E -01

3.7E-01

Production/Consumption factors:
Produce: 1.4
Milk: 8.4
Meat: 2.1

FRACTION OF POPULATION RECEIVING AN INDICATED AVERAGE TOTAL-BODY DOSE COMMITMENT FROM AIRBORNE PATHWAYS

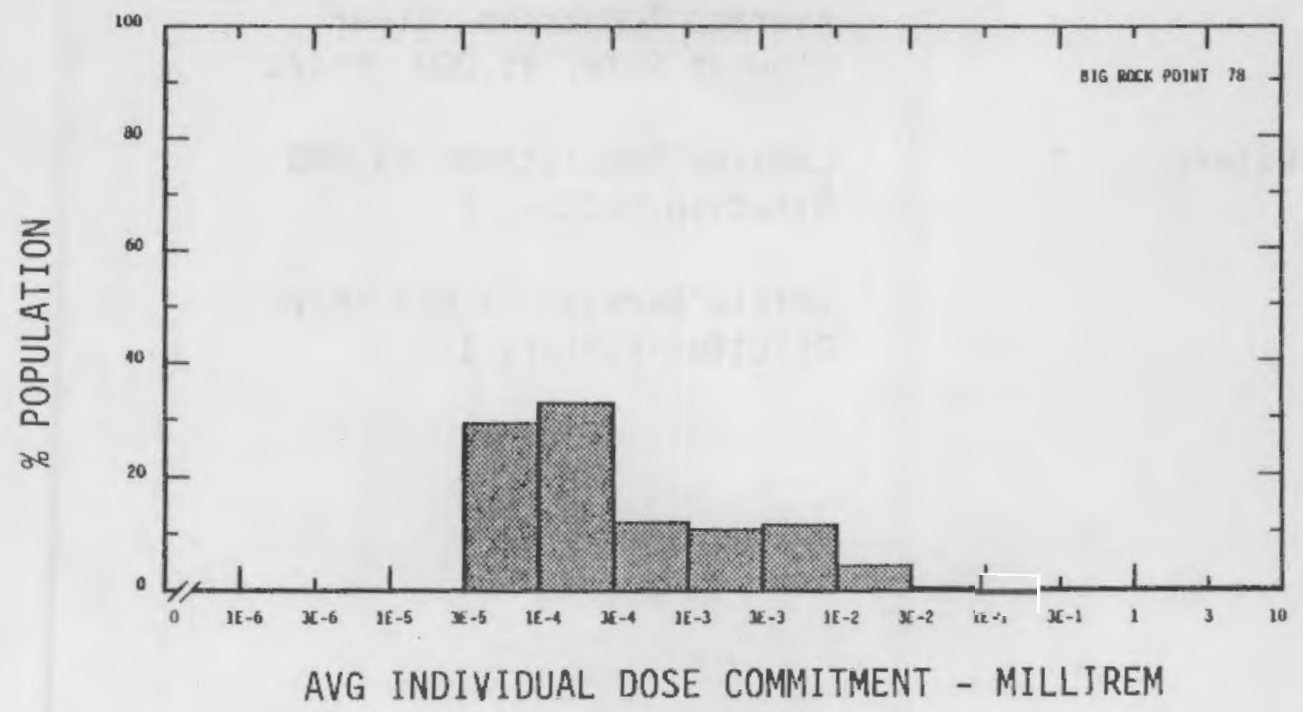


Location: $\quad$ N $34.70360 \quad$ W 87.12110

POPULATION DATA

Total Population Within 20-to-80-km Region: 6.855

Major Metropolitan Centers Within Region:

\begin{tabular}{lrrrr}
\multicolumn{1}{c}{ Center } & Population & \multicolumn{2}{c}{ Location } \\
\cline { 2 - 4 } & 41,000 & $16 \mathrm{~km}$ & SE \\
Decatur & 150,000 & $48 \mathrm{~km}$ & E
\end{tabular}

SITE SPECIFIC DATA - AIRBORNE PATHWAYS

Average Annual State Production

of Crops and Animal Products

In $80-\mathrm{km}$ Radius Circle

Regional Productivity Factor:

Animal Grazing Factor:
Veg: $1.7 E 7$ kilogram

Milk: 5.7E7 liter

Meat: $8.6 \mathrm{E} 7 \mathrm{kilogram}$

1

0.7

Meteorology Period of Record: 1 JAN 74 - 31 DEC 75 Recovery: 94\%

\section{SITE SPECIFIC DATA - WATERBORNE PATHWAYS}

Average Tennessee River

Flow at Site: $45,000 \mathrm{ft} 3 / \mathrm{s}$

Drinking Water:

Exposed Population: 23,000

Dilution Factor: 1

Fish:

Edible Harvest: $1.6 E 6 \mathrm{~kg} / \mathrm{yr}$

Dilution Factor: 1 
POPULATION DOSE-COMMITMENT ESTIMATES AND

AVERAGE INDIVIDUAL DOSE-COMMITMENT HISTOGRAM FOR

BROWNS FERRY 1, 2 AND 3

Dose Commitments (person-rem) from Liquid Pathways

Infant

Total Body

GI-LLI

Thyroid

Bone Liver

Child

3. $9 \mathrm{E}-05$

4.2E-05

1. 5E -03

1.1E-04

1.4E-04

Teen

6.3E-02

1. $0 \mathrm{E}-01$

1. $9 \mathrm{E}-02$

1.7E-01

2. $2 E-01$

Adult

8.0E-02

2.1E-01

9. $0 \mathrm{E}-03$

1. $0 \mathrm{E}-01$

1. $9 \mathrm{E}-01$

7. 5E-01

1. $9 \mathrm{E}+00$

6. 0E-02

6.1E-01

1. $2 \mathrm{E}+00$

TOTAL

8.9E-01

2. $2 E+00$

8. $9 \mathrm{E}-02$

8. $9 \mathrm{E}-01$

1. $6 \mathrm{E}+00$

Dose Commitments (person-rem) from Airborne Pathways Total Body GI-LLI Thyroid Bone Liver Lung

$\begin{array}{lllllll}\text { Infant } & 1.9 \mathrm{E}-02 & 1.9 \mathrm{E}-02 & 6.1 \mathrm{E}-02 & 1.9 \mathrm{E}-02 & 2.0 \mathrm{E}-02 & 2.5 \mathrm{E}-02 \\ \text { Child } & 2.1 \mathrm{E}-01 & 2.1 \mathrm{E}-01 & 4.8 \mathrm{E}-01 & 2.2 \mathrm{E}-01 & 2.2 \mathrm{E}-01 & 2.9 \mathrm{E}-01 \\ \text { Teen } & 1.6 \mathrm{E}-01 & 1.6 \mathrm{E}-01 & 2.7 \mathrm{E}-01 & 1.6 \mathrm{E}-01 & 1.6 \mathrm{E}-01 & 2.6 \mathrm{E}-01 \\ \text { Adult } & 9.4 \mathrm{E}-01 & 9.5 \mathrm{E}-01 & 1.4 \mathrm{E}+00 & 9.4 \mathrm{E}-01 & 9.4 \mathrm{E}-01 & 1.3 \mathrm{E}+00 \\ \text { TOTAL } & 1.3 \mathrm{E}+00 & 1.3 \mathrm{E}+00 & 2.2 \mathrm{E}+00 & 1.3 \mathrm{E}+00 & 1.3 \mathrm{E}+00 & 1.8 \mathrm{E}+00\end{array}$

Production/Consumption factors:

Produce: $<1$ Milk: $<1 \quad$ Meat: 1.6

FRACTION OF POPULATION RECEIVING AN INDICATED AYERAGE TOTAL-BODY DOSE COMMITMENT FROM AIRBORNE PATHWAYS

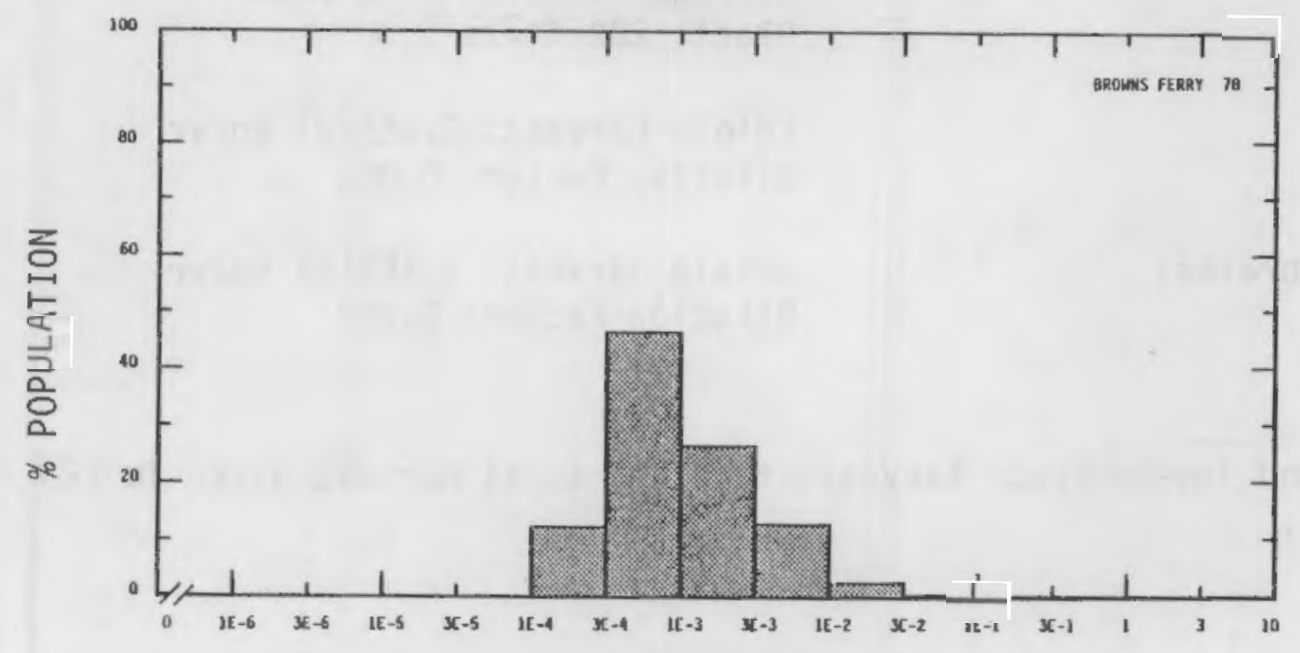

AVG INDIVIDUAL DOSE COMMITMENT - MILLIREM 
Location: N 33.95860 W 78.02080

POPULATION DATA

Total Population Within 20-to-80-km Region: $1.9 E 5$

Major Metropolitan Centers Within Region:

\begin{tabular}{lcl} 
Center & Population & \multicolumn{1}{l}{ Location } \\
Wilmington & 51,000 & $32 \mathrm{~km} \quad \mathrm{~N}$
\end{tabular}

SITE SPECIFIC DATA - AIRBORNE PATHWAYS

Average Annual State Production

of Crops and Animal Products

In 80-km Radius Circle

Regional Productivity Factor:

Animal Grazing Factor:
Veg: 2.6E7 kilogram

Milk: 1.0E8 liter

Meat: $5.8 E 7$ kilogram

0.3

0.7

Meteorology Period of Record: 1 JAN 75 - 31 DEC 75 Recovery: 93\%

Average Dilution Flow from

Plant: $202 \mathrm{ft} 3 / \mathrm{s}$

Fish:

Edible Harvest: 2.1E5(a) $\mathrm{kg} / \mathrm{yr}$ Dilution Factor: 0.001

Invertebrates:
Edible Harvest: $1.1 E 5(a) \mathrm{kg} / \mathrm{yr}$

Dilution Factor: 0.002

(a) Fish and invertebrate harvests together total harvest given in FES (1973). 
POPULATION DOSE-COMMITMENT ESTIMATES AND

AVERAGE INDIVIDUAL DOSE-COMMITMENT HISTOGRAM FOR

BRUNSWICK 1 AND 2

Dose Commitments (person-rem) from Liquid Pathways

Total Body GI-LLI Thyroid Bone Liver

$\begin{array}{llllll}\text { Infant } & 0.0 \mathrm{E}+00 & 0.0 \mathrm{E}+00 & 0.0 \mathrm{E}+00 & 0.0 \mathrm{E}+00 & 0.0 \mathrm{E}+00 \\ \text { Child } & 7.7 \mathrm{E}-02 & 1.8 \mathrm{E}+02 & 4.4 \mathrm{E}-04 & 2.6 \mathrm{E}-01 & 1.1 \mathrm{E}-01 \\ \text { Teen } & 5.4 \mathrm{E}-02 & 3.6 \mathrm{E}+02 & 3.1 \mathrm{E}-04 & 1.6 \mathrm{E}-01 & 9.9 \mathrm{E}-02 \\ \text { Adult } & 3.2 \mathrm{E}-01 & 3.2 \mathrm{E}+03 & 2.0 \mathrm{E}-03 & 9.9 \mathrm{E}-01 & 6.1 \mathrm{E}-01 \\ \text { TOTAL } & 4.5 \mathrm{E}-01 & 3.7 \mathrm{E}+03 & 2.8 \mathrm{E}-03 & 1.4 \mathrm{E}+00 & 8.2 \mathrm{E}-01\end{array}$

Dose Commitments (person-rem) from Airborne Pathways

Total Body GI-LLI Thyroid Bone Liver Lung

$\begin{array}{lllllll}\text { Infant } & 2.7 E-02 & 2.7 E-02 & 6.1 E-02 & 2.7 E-02 & 2.7 E-02 & 2.7 E-02 \\ \text { Child } & 3.0 E-01 & 2.9 E-01 & 5.1 E-01 & 3.0 E-01 & 3.0 E-01 & 3.1 E-01 \\ \text { Teen } & 2.2 E-01 & 2.2 E-01 & 3.0 E-01 & 2.2 E-01 & 2.2 E-01 & 2.3 E-01 \\ \text { Adult } & 1.3 E+00 & 1.3 E+00 & 1.6 E+00 & 1.3 E+00 & 1.3 E+00 & 1.4 E+00 \\ \text { TOTAL } & 1.8 E+00 & 1.8 E+00 & 2.5 E+00 & 1.8 E+00 & 1.8 E+00 & 1.9 E+00\end{array}$

Production/Consumption factors:

Produce: <1 Milk: 1.2 Meat: 1.1

FRACTION OF POPULATION RECEIVING AN INDICATED AVERAGE TOTAL-BODY DOSE COMMITMENT FROM AIRBORNE PATHWAYS

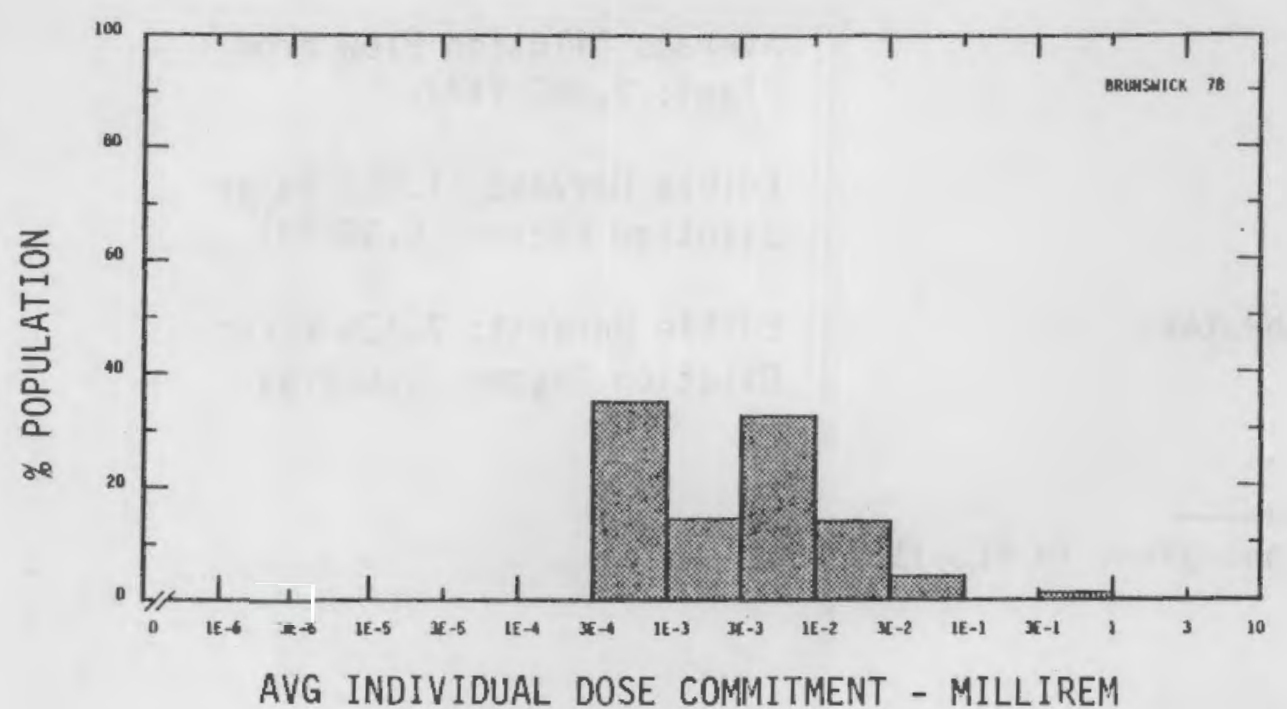


Site: CALVERT CLIFFS LUSBY, MARYLAND

Location: N $38.4272^{\circ}$ W 76.42610

POPULATION DATA

Total Population Within 20-to-80-km Region: 2.4E6

Major Metropolitan Centers Within Region:

Center Population Location

Washington, DC SMSA (3/4) 2,300,000 $75 \mathrm{~km} \quad \mathrm{NW}$

Cambridge $12,000 \quad 32 \mathrm{~km}$ ENE

Annapolis $\quad 31,000 \quad 61 \mathrm{~km} \mathrm{~N}$

SITE SPECIFIC DATA - AIRBORNE PATHWAYS

Average Annual State Production

of Crops and Animal Products

In 80-km Radius Circle

Regional Productivity Factor:

Animal Grazing Factor:
Veg: $4.5 E 7$ kilogram

Milk: 5.0E8 liter

Meat: $6.2 \mathrm{E} 7 \mathrm{kilogram}$

0.6

0.6

Meteorology Period of Record: 1 JAN 75 - 31 DEC 75 Recovery: $96 \%$

SITE SPECIFIC DATA - WATERBORNE PATHWAYS

Average Dilution Flow from

P1ant: $2,600 \mathrm{ft}^{3} / \mathrm{s}$

Fish:

Edible Harvest: $1.0 \mathrm{E} 7 \mathrm{~kg} / \mathrm{yr}$ Dilution Factor: $0.062($ a)

Invertebrates:

Edible Harvest: $7.4 \mathrm{E} 6 \mathrm{~kg} / \mathrm{yr}$

Dilution Factor: 0.062 (a)

(a) Dilutions given in FES (1973). 
POPULATION DOSE-COMMITMENT ESTIMATES AND

AVERAGE INDIVIDUAL DOSE-COMMITMENT HISTOGRAM FOR

CALVERT CLIFFS 1 AND 2

Dose Commitments (person-rem) from Liquid Pathways

Total Body

GI-LLI

Thyroid

Bone

Liver

Infant

$0.0 \mathrm{E}+00$

$0.0 E+00$

$0.0 \mathrm{E}+00$

$0.0 E+00$

$0.0 \mathrm{E}+00$

Child

2.1E-01

$1.6 \mathrm{E}+00$

5.3E-01

2.8E-01

4.1E-01

Teen

Adult

$1.9 E-01$

3. $3 E+00$

3.7E-01

1.7E-01

3. $5 E-01$

1. $4 \mathrm{E}+00$

$2.8 \mathrm{E}+01$

2. $5 E+00$

1. $0 \mathrm{E}+00$

$2.1 E+00$

TOTAL

1. $9 E+00$

3. $3 \mathrm{E}+01$

3. $4 \mathrm{E}+00$

$1.5 \mathrm{E}+00$

2. $9 E+00$

Dose Commitments (person-rem) from Airborne Pathways

Total Body GI-LLI Thyroid Bone Liver Lung

$\begin{array}{lllllll}\text { Infant } & 1.1 \mathrm{E}-02 & 1.0 \mathrm{E}-02 & 1.6 \mathrm{E}-01 & 1.1 \mathrm{E}-02 & 1.1 \mathrm{E}-02 & 1.1 \mathrm{E}-02 \\ \text { Child } & 1.2 \mathrm{E}-01 & 1.2 \mathrm{E}-01 & 9.2 \mathrm{E}-01 & 1.2 \mathrm{E}-01 & 1.2 \mathrm{E}-01 & 1.3 \mathrm{E}-01 \\ \text { Teen } & 8.5 \mathrm{E}-02 & 8.4 \mathrm{E}-02 & 4.0 \mathrm{E}-01 & 8.5 \mathrm{E}-02 & 8.6 \mathrm{E}-02 & 1.0 \mathrm{E}-01 \\ \text { Adu1t } & 5.1 \mathrm{E}-01 & 5.1 \mathrm{E}-01 & 1.5 \mathrm{E}+00 & 5.1 \mathrm{E}-01 & 5.1 \mathrm{E}-01 & 5.7 \mathrm{E}-01 \\ \text { TOTAL } & 7.2 \mathrm{E}-01 & 7.2 \mathrm{E}-01 & 3.0 \mathrm{E}+00 & 7.3 \mathrm{E}-01 & 7.3 \mathrm{E}-01 & 8.1 \mathrm{E}-01\end{array}$

Production/Consumption factors:

Produce: <1 Milk: <1 Meat: <1

FRACTION OF POPULATION RECEIVING AN INDICATED AVERAGE TOTAL-BODY DOSE COMMITMENT FROM AIRBORNE PATHWAYS

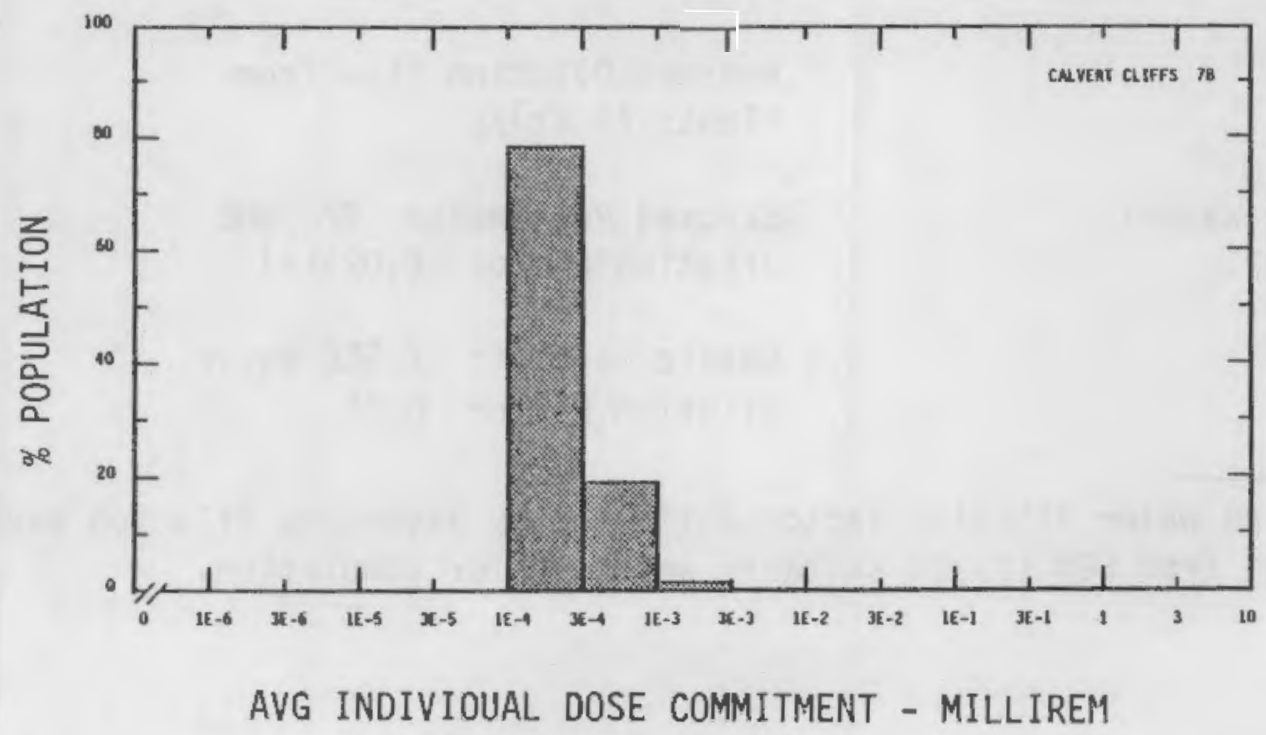




Location: N $41.9742 \quad$ W 86.56670

POPULATION DATA

Total Population Within 20-to-80-km Region: $1.1 \mathrm{E} 6$

\begin{tabular}{lcccc}
\multicolumn{3}{c}{ Major Metropolitan Centers Within Region: } \\
\multicolumn{1}{c}{ Center } & Population & \multicolumn{1}{c}{ Location } \\
Gary SMSA & 650,000 & & \\
South Bend SMSA & 290,000 & $40 \mathrm{~km}$ & SW \\
Benton Harbor & 17,000 & $42 \mathrm{~km}$ & SE \\
Sento & $18 \mathrm{~km}$ & NE
\end{tabular}

SITE SPECIFIC DATA - AIRBORNE PATHWAYS

Average Annual State Production

of Crops and Animal Products

In $80-\mathrm{km}$ Radius Circle

Regional Productivity Factor:

Animal Grazing Factor:
Veg: 1.1 E8 kilogram

Milk: 2.3E8 1iter

Meat: $1.9 \mathrm{E} 8$ kilogram

0.6

0.5

Meteorology Period of Record: 1 MAY 75 - 31 APR 76 Recovery: 95\%

SITE SPECIFIC DATA - WATERBORNE PATHWAYS

Average Dilution $\mathrm{Fl}$ ow from

P1ant: $77 \mathrm{ft} 3 / \mathrm{s}$

Drinking Water:

Exposed Population: 270,000

Dilution Factor: 0.025(a)

Fish:

Edible Harvest: $1.5 \mathrm{E} 6 \mathrm{~kg} / \mathrm{yr}$

Dilution Factor: 0.01

(a) Drinking water dilution factor estimated by averaging dilution factors derived from FES (1973) suitably weighted for population. 
POPULATION DOSE-COMMITMENT ESTIMATES AND

AVERAGE INDIVIDUAL DOSE-COMMITMENT HISTOGRAM FOR

COOK 1 AND 2

Dose Commitments (person-rem) from Liquid Pathways

Total Body GI-LLI Thyroid Bone Liver

$\begin{array}{llllll}\text { Infant } & 6.0 E-02 & 5.0 E-02 & 9.9 E-02 & 9.5 E-02 & 1.8 E-01 \\ \text { Child } & 2.2 E+00 & 1.1 E+00 & 9.0 E-01 & 7.4 E+00 & 9.6 E+00 \\ \text { Teen } & 3.1 E+00 & 1.4 E+00 & 3.2 E-01 & 4.1 E+00 & 7.3 E+00 \\ \text { Adult } & 3.4 E+01 & 1.2 E+01 & 2.5 E+00 & 2.4 E+01 & 4.5 E+01 \\ \text { TOTAL } & 3.9 E+01 & 1.5 E+01 & 3.8 E+00 & 3.6 E+01 & 6.2 E+01\end{array}$

Dose Commitments (person-rem) from Airborne Pathways

Total Body GI-LLI Thyroid Bone Liver Lung

$\begin{array}{lllllll}\text { Infant } & 7.7 \mathrm{E}-03 & 7.7 \mathrm{E}-03 & 1.4 \mathrm{E}-02 & 7.6 \mathrm{E}-03 & 7.7 \mathrm{E}-03 & 8.4 \mathrm{E}-03 \\ \text { Child } & 8.6 \mathrm{E}-02 & 8.7 \mathrm{E}-02 & 1.2 \mathrm{E}-01 & 8.5 \mathrm{E}-02 & 8.5 \mathrm{E}-02 & 9.7 \mathrm{E}-02 \\ \text { Teen } & 6.2 \mathrm{E}-02 & 6.4 \mathrm{E}-02 & 7.7 \mathrm{E}-02 & 6.2 \mathrm{E}-02 & 6.2 \mathrm{E}-02 & 7.6 \mathrm{E}-02 \\ \text { Adult } & 3.8 \mathrm{E}-01 & 3.9 \mathrm{E}-01 & 4.3 \mathrm{E}-01 & 3.7 \mathrm{E}-01 & 3.8 \mathrm{E}-01 & 4.3 \mathrm{E}-01 \\ \text { TOTAL } & 5.3 \mathrm{E}-01 & 5.5 \mathrm{E}-01 & 6.4 \mathrm{E}-01 & 5.3 \mathrm{E}-01 & 5.3 \mathrm{E}-01 & 6.1 \mathrm{E}-01\end{array}$

Production/Consumption factors:

Produce: $<1 \quad$ Milk: $<1 \quad$ Meat: 1.3

FRACTION OF POPULATION RECEIVING AN INDICATED AVERAGE TOTAL-BODY DOSE COMMITMENT FROM AIRBORNE PATHWAYS

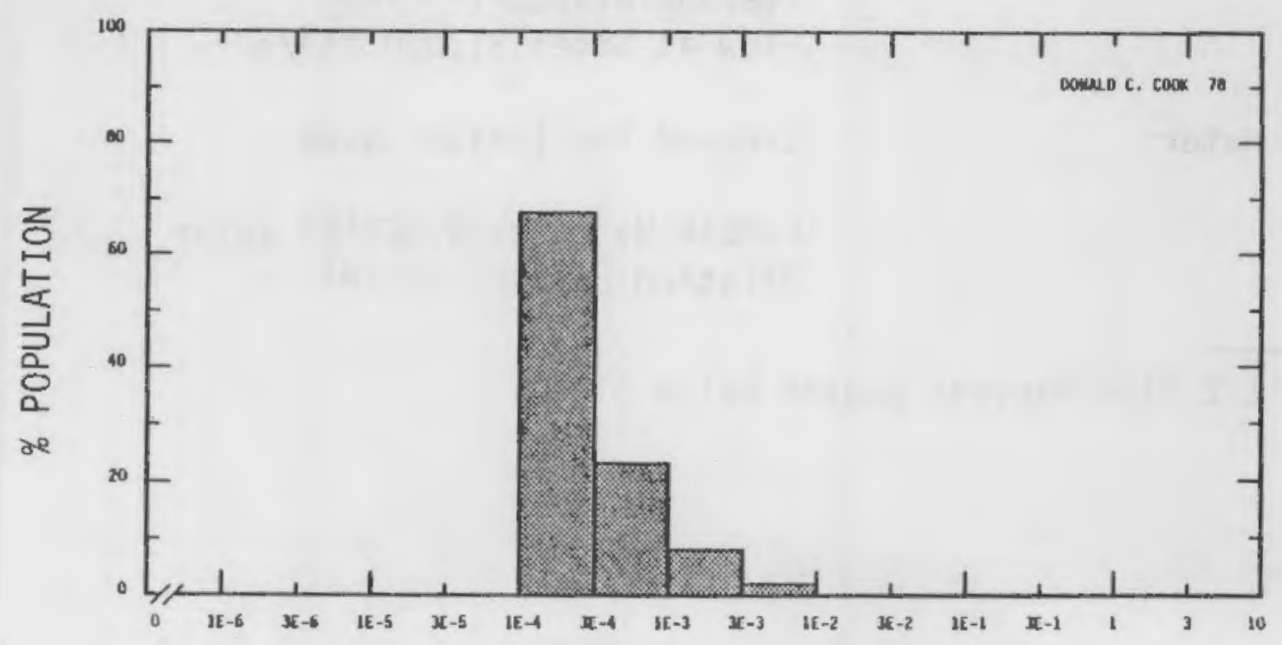

AVG INDIVIDUAL DOSE COMMITMENT - MILLIREM 
Site: COOPER STATION

NEMAHA COUNTY, NEBRASKA

Location: N $40.36140 \quad$ W 95.63780

POPULATION DATA

Total Population Within 20-to-80-km Region: $1.8 \mathrm{E} 5$

Major Metropolitan Centers Within Region:

Center Population Location

$\begin{array}{llll}\text { Shenandoah } & 6,300 & 58 \mathrm{~km} & \text { NNE }\end{array}$

SITE SPECIFIC DATA - AIRBORNE PATHWAYS

Average Annual State Production

of Crops and Animal Products

In $80-\mathrm{km}$ Radius Circle

Regional Productivity Factor:

Animal Grazing Factor:
Veg: 9.7E7 kilogram

Milk: 7.2E7 liter

Meat: 2.0E8 kilogram

1

0.6

Meteorology Period of Record: 1 MAR 70 - 31 DEC 75 Recovery: $89 \%$

SITE SPECIFIC DATA - WATERBORNE PATHWAYS

Average Missouri River

Flow at Site: $31,000 \mathrm{ft}^{3} / \mathrm{s}$

Drinking water:

Exposed Population: None

Fish:

Edible Harvest: $5.0 \mathrm{E}_{3}(\mathrm{a}) \mathrm{kg} / \mathrm{yr}$

Dilution Factor: $0.5(\mathrm{a})$

(a) Assumes $1 / 2$ fish harvest caught below plant. 
POPULATION DOSE-COMMITMENT ESTIMATES AND

AVERAGE INDIVIOUAL DOSE-COMMITMENT HISTOGRAM FOR

COOPER STATION

Dose Commitments (person-rem) from Liquid Pathways

Total Body GI-LLI Thyroid Bone

$\begin{array}{llllll}\text { Infant } & 0.0 E+00 & 0.0 E+00 & 0.0 E+00 & 0.0 E+00 & 0.0 E+00 \\ \text { Child } & 5.2 E-04 & 5.1 E-05 & 4.2 E-05 & 2.4 E-03 & 2.9 E-03 \\ \text { Teen } & 1.0 E-03 & 1.1 E-04 & 3.0 E-05 & 1.4 E-03 & 2.5 E-03 \\ \text { Adult } & 1.1 E-02 & 9.3 E-04 & 2.0 E-04 & 8.3 E-03 & 1.5 E-02 \\ \text { TOTAL } & 1.2 E-02 & 1.1 E-03 & 2.7 E-04 & 1.2 E-02 & 2.0 E-02\end{array}$

Dose Commitments (person-rem) from Airborne Pathways

Total Body GI-LLI Thyroid Bone Liver Lung

$\begin{array}{lllllll}\text { Infant } & 5.9 E-04 & 5.9 E-04 & 1.0 E-03 & 5.9 E-04 & 6.0 E-04 & 6.1 E-04 \\ \text { Child } & 6.7 E-03 & 6.7 E-03 & 1.1 E-02 & 6.7 E-03 & 6.7 E-03 & 6.9 E-03 \\ \text { Teen } & 4.9 E-03 & 4.9 E-03 & 6.5 E-03 & 4.9 E-03 & 4.9 E-03 & 5.2 E-03 \\ \text { Adult } & 2.9 E-02 & 2.9 E-02 & 3.5 E-02 & 2.9 E-02 & 2.9 E-02 & 3.1 E-02 \\ \text { TOTAL } & 4.2 E-02 & 4.1 E-02 & 5.4 E-02 & 4.1 E-02 & 4.2 E-02 & 4.3 E-02\end{array}$

Production/Consumption factors:

Produce: $2.8 \quad$ Milk: 3.1 Meat: 14.0

FRACTION OF POPULATION RECEIVING AN INDICATED AVERAGE

TOTAL-BODY DOSE COMMITMENT FROM AIRBORNE PATHWAYS

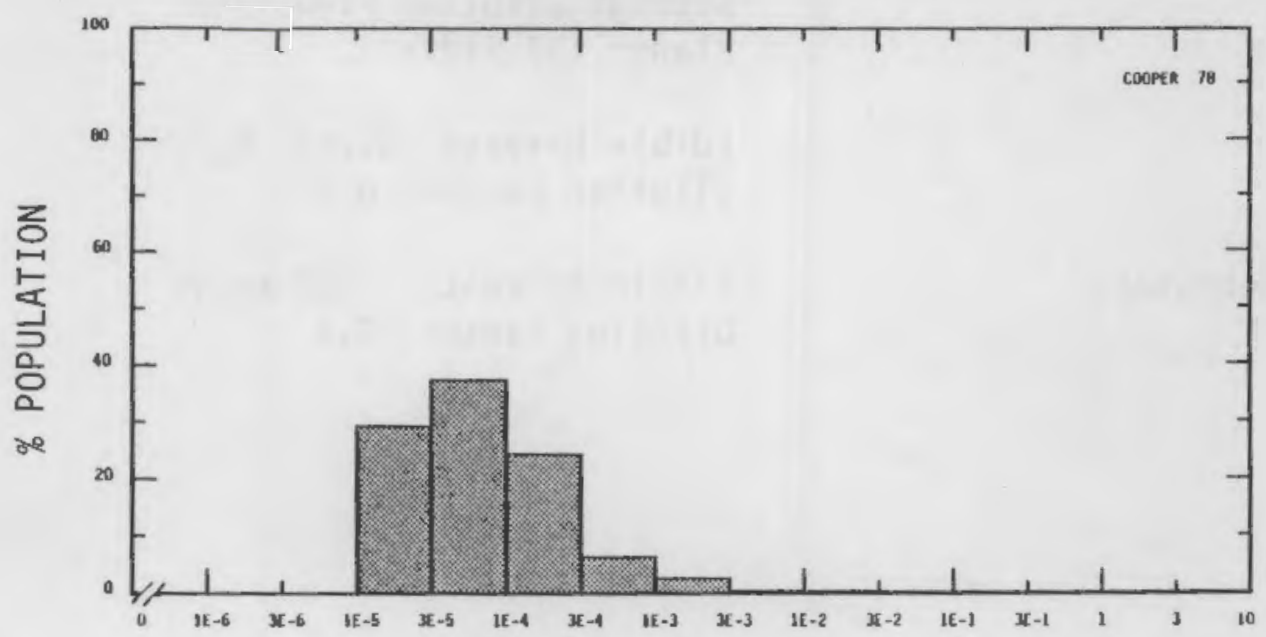

AVG INDIVIDUAL DOSE COMMITMENT - MILLIREM 
Site: CRYSTAL RIVER

Location: $\quad$ N 28.95670
CRYSTAL RIVER, FLORIDA

W 82.69800

POPULATION DATA

Total Population Within 20-to-80-km Region: 2.3E5

Major Metropolitan Centers Within Region:

\begin{tabular}{lll} 
Center & Population & \multicolumn{2}{l}{ Location } \\
Ocala & 28,000 & $63 \mathrm{~km}$ ENE
\end{tabular}

SITE SPECIFIC DATA - AIRBORNE PATHWAYS

Average Annual State Production

of Crops and Animal Products

In $80-\mathrm{km}$ Radius Circle

Regional Productivity Factor:

Animal Grazing Factor:
Veg: $2.8 \mathrm{E} 7 \mathrm{kilogram}$

Milk: 1.1E8 liter

Meat: $7.2 E 7$ kilogram

0.5

1

Meteorology Period of Record: 1 JAN 75 - 31 DEC 75 Recovery: 93\%

SITE SPECIFIC DATA - WATERBORNE PATHWAYS

Average 0ilution Flow from

Plant: $132 \mathrm{ft}^{3} / \mathrm{s}$

Fish:

Edible Harvest: $3.2 \mathrm{E} 5 \mathrm{~kg} / \mathrm{yr}$

Dilution Factor: 0.1

Invertebrates:

Edible Harvest: $1.85 .5 \mathrm{~kg} / \mathrm{yr}$

Dilution Factor: 0.1 
POPULATION DOSE-COMMITMENT ESTIMATES AND

AVERAGE INDIVIDUAL DOSE-COMMITMENT HISTOGRAM FOR

CRYSTAL RIVER

Dose Commitments (person-rem) from Liquid Pathways

Total Body GI-LLI Thyroid Bone Liver

Infant

$0.0 \mathrm{E}+00$

$0.0 \mathrm{E}+00$

$0.0 \mathrm{E}+00$

$0.0 \mathrm{E}+00$

$0.0 \mathrm{E}+00$

Child

Teen

Aduit

4.1E-02

3. $8 \mathrm{E}-02$

$1.4 \mathrm{E}-02$

3. $\mathrm{OE}-02$

6. $6 \mathrm{E}-02$

2.9E-02

7. $9 \mathrm{E}-02$

1. $0 \mathrm{E}-02$

2.1E-02

6.0E-02

1.8E-01

6. $9 \mathrm{E}-01$

6.7E-02

1. $4 \mathrm{E}-01$

3. $7 \mathrm{E}-01$

TOTAL

2. $5 \mathrm{E}-01$

8.1E-01

9.1E-02

1.9E-01

5. $0 \mathrm{E}-01$

Dose Commitments (person-rem) from Airborne Pathways

Total Body GI-LLI Thyroid Bone Liver Lung

Infant

$5.6 \mathrm{E}-04$

$5.6 \mathrm{E}-04$

8. $9 \mathrm{E}-04$

$5.5 \mathrm{E}-04$

$5.7 \mathrm{E}-04$

$5.8 \mathrm{E}-04$

Child

$6.3 \mathrm{E}-03$

$6.3 \mathrm{E}-03$

$8.8 \mathrm{E}-03$

$6.1 \mathrm{E}-03$

$6.4 \mathrm{E}-03$

$6.6 \mathrm{E}-03$

Teen

4. $6 \mathrm{E}-03$

4. $5 \mathrm{E}-03$

5.7E-03

4. $4 \mathrm{E}-03$

4. $6 \mathrm{E}-03$

5. $0 \mathrm{E}-03$

Adult

2.7E-02

2.7E-02

3. $2 \mathrm{E}-02$

2.7E-02

2. $8 \mathrm{E}-02$

2. $9 \mathrm{E}-02$

TOTAL.

3. $9 \mathrm{E}-02$

3. $9 \mathrm{E}-02$

4.7E-02

3. $8 \mathrm{E}-02 \quad 3.9 \mathrm{E}-02$

4.1E-02

Production/Consumption factors:

Produce: <1

Milk: 1.9

Meat: 2.0

FRACTION OF POPULATION RECEIVING AN INDICATED AVERAGE TOTAL-BDDY DOSE COMMITMENT FROM AIRBORNE PATHWAYS

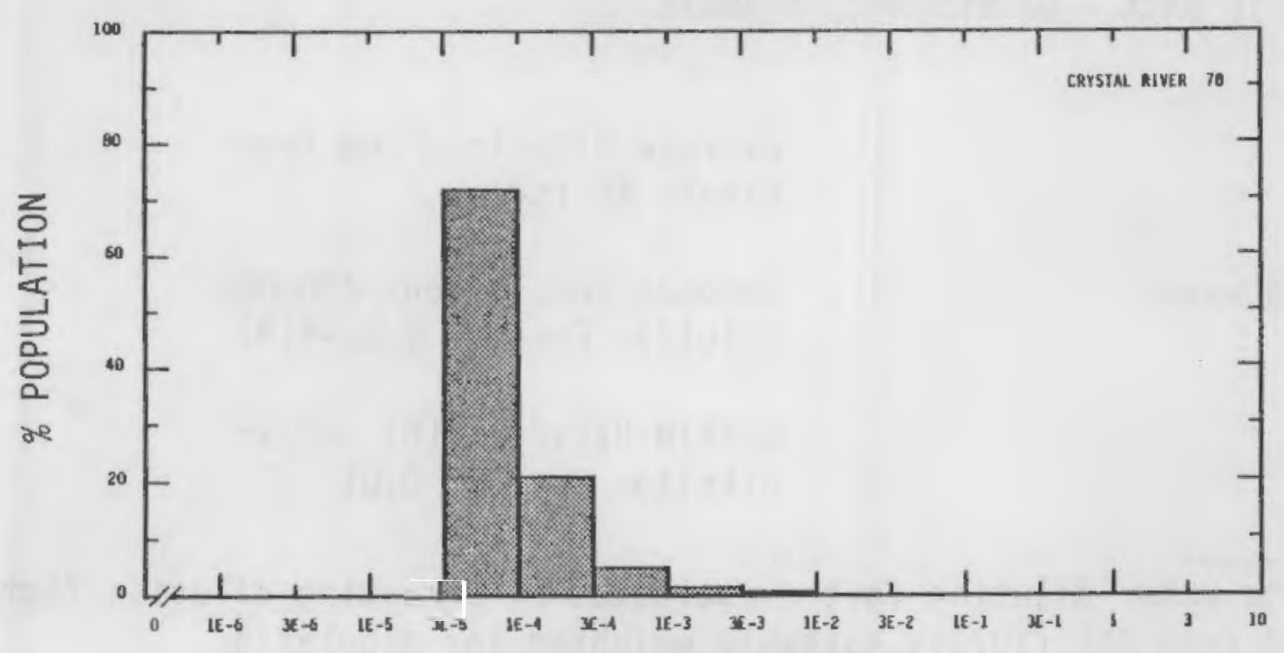

AVG INDIVIDUAL DOSE COMMITMENT - MILLIREM 


Location: $\quad$ N $41.59470 \quad$ W 83.08640

POPULATION DATA

Total Population Within 20-to-80-km Region: $1.8 \mathrm{E} 6$

Major Metropolitan Centers Within Region:

\begin{tabular}{lcccc} 
Center & Population & & \multicolumn{2}{c}{ Location } \\
\cline { 2 - 5 } Toledo & 390,000 & & $37 \mathrm{~km}$ & WNW \\
Sandusky & 33,000 & $35 \mathrm{~km}$ & ESE \\
Findlay & 36,000 & $77 \mathrm{~km}$ & SW \\
Dearborn & 110,000 & $80 \mathrm{~km}$ & $\mathrm{~N}$
\end{tabular}

SITE SPECIFIC DATA - AIRBORNE PATHWAYS

Average Annual State Production

of Crops and Animal Products

In 80-km Radius Circle

Regional Productivity Factor:

Animal Grazing Factor:
Veg: $6.9 \mathrm{E} 7 \mathrm{kilogram}$

Milk: $3.7 E 8$ liter

Meat: $1.2 \mathrm{E} 8 \mathrm{kilogram}$

0.5

0.5

Meteorology Period of Record: 4 AUG 74 - 3 AUG 76 Recovery: 99\%

SITE SPECIFIC DATA - WATERBORNE PATHWAYS

Average Dilution Flow from

Plant: $48 \mathrm{ft} 3 / \mathrm{s}$

Orinking Water:

Exposed Population: 450,000

Dilution Factor: $2.0 \mathrm{E}-4(\mathrm{a})$

Fish:

Edible Harvest: (b) $\mathrm{kg} / \mathrm{yr}$

Dilution Factor: 0.01

(a) Orinking water dilution factor estimated by averaging dilution factor derived from FES (1973), suitably weighted for population.

(b) No fish catch data given in FES (1973); thus, generic consumption rates used (Table A-1). 
POPULATION DOSE-COMMITMENT ESTIMATES AND AVERAGE INDIVIDUAL DOSE-COMMITMENT HISTOGRAM FOR DAVIS-BESSE

Dose Commitments (person-rem) from Liquid Pathways

Total Body GI-LLI Thyroid Bone Liver

$\begin{array}{llllll}\text { Infant } & 3.0 \mathrm{E}-04 & 2.8 \mathrm{E}-04 & 2.9 \mathrm{E}-04 & 1.1 \mathrm{E}-04 & 2.7 \mathrm{E}-04 \\ \text { Child } & 5.3 \mathrm{E}-02 & 8.5 \mathrm{E}-02 & 1.1 \mathrm{E}-02 & 1.6 \mathrm{E}-01 & 8.2 \mathrm{E}-02 \\ \text { Teen } & 5.4 \mathrm{E}-02 & 1.7 \mathrm{E}-01 & 7.8 \mathrm{E}-03 & 1.2 \mathrm{E}-01 & 6.9 \mathrm{E}-02 \\ \text { Adult } & 4.7 \mathrm{E}-01 & 1.4 \mathrm{E}+00 & 6.1 \mathrm{E}-02 & 8.3 \mathrm{E}-01 & 4.3 \mathrm{E}-01 \\ \text { TOTAL } & 5.8 \mathrm{E}-01 & 1.7 \mathrm{E}+00 & 8.0 \mathrm{E}-02 & 1.1 \mathrm{E}+00 & 5.8 \mathrm{E}-01\end{array}$

Dose Commitments (person-rem) from Airborne Pathways

Total Body GI-LLI Thyroid Bone Liver Lung

$\begin{array}{lllllll}\text { Infant } & 3.3 \mathrm{E}-04 & 3.3 \mathrm{E}-04 & 5.2 \mathrm{E}-04 & 1.9 \mathrm{E}-04 & 3.4 \mathrm{E}-04 & 3.9 \mathrm{E}-04 \\ \text { Child } & 3.9 \mathrm{E}-03 & 3.9 \mathrm{E}-03 & 5.0 \mathrm{E}-03 & 2.2 \mathrm{E}-03 & 4.0 \mathrm{E}-03 & 4.8 \mathrm{E}-03 \\ \text { Teen } & 2.7 \mathrm{E}-03 & 2.7 \mathrm{E}-03 & 3.0 \mathrm{E}-03 & 1.5 \mathrm{E}-03 & 2.7 \mathrm{E}-03 & 3.8 \mathrm{E}-03 \\ \text { Adult } & 1.5 \mathrm{E}-02 & 1.5 \mathrm{E}-02 & 1.6 \mathrm{E}-02 & 9.2 \mathrm{E}-03 & 1.5 \mathrm{E}-02 & 1.9 \mathrm{E}-02 \\ \text { TOTAL } & 2.2 \mathrm{E}-02 & 2.2 \mathrm{E}-02 & 2.5 \mathrm{E}-02 & 1.3 \mathrm{E}-02 & 2.2 \mathrm{E}-02 & 2.8 \mathrm{E}-02\end{array}$

Production/Consumption factors:

$\begin{array}{lll}\text { Produce: }<1 & \text { Milk: }<1 & \text { Meat: }<1\end{array}$

FRACTION OF POPULATION RECEIVING AN INDICATED AVERAGE TOTAL-BODY DOSE COMMITMENT FROM AIRBORNE PATHWAYS

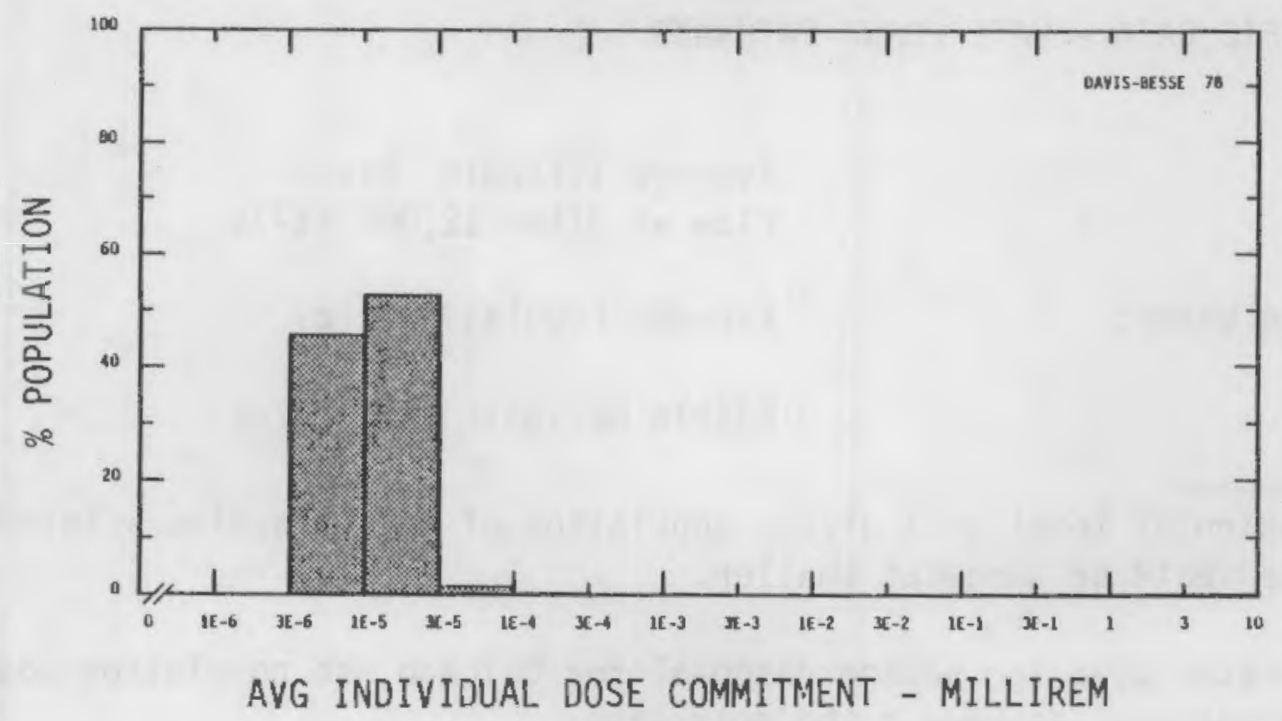


Site: DRESDEN

Location: $\quad$ N $41.38970 \quad$ W 88.27140

POPULATION DATA

Total Population Within 20-to-80-km Region: 6.4E6

Major Metropolitan Centers Within Region:

\begin{tabular}{|c|c|c|c|}
\hline Center & Population & \multicolumn{2}{|c|}{ Location } \\
\hline Chicago SMSA & $7,000,000(a)$ & $72 \mathrm{~km}$ & NE \\
\hline Gary-Hanmond-E.Chicago-SMSA & $640,000(a)$ & $80 \mathrm{~km}$ & ENE \\
\hline Joliet & 81,000 & $24 \mathrm{~km}$ & NE \\
\hline Aurora & 75,000 & $43 \mathrm{~km}$ & $\mathrm{~N}$ \\
\hline Elgin & 56,000 & $74 \mathrm{~km}$ & N \\
\hline
\end{tabular}

SITE SPECIFIC DATA - AIRBORNE PATHWAYS

Average Annual State Production

of Crops and Animal Products

In $80-\mathrm{km}$ Radius Circle

Regional Productivity Factor:

Animal Grazing Factor:
Veg: $1.1 \mathrm{E} 8 \mathrm{ki}$ logram

Milk: $1.8 \mathrm{E} 8$ liter

Meat: $1.9 \mathrm{E} 8$ kilogram

1

0.5

Meteorology Period of Record: 1 JAN $74-31$ JAN 75 Recovery: $77 \%$

SITE SPECIFIC DATA - WATERBORNE PATHWAYS

Average Illinois River

Flow at Site: $12,000 \mathrm{ft}^{3} / \mathrm{s}$

Drinking Water:

Exposed Population: (b)

Fish:

Edible Harvest: (b) $\mathrm{kg} / \mathrm{yr}$

(a) Population of total SMSA given; population of SMSA fraction within $80 \mathrm{~km}$ of site would be somewhat smaller.

(b) River water used for sewage disposal for Chicago, so population doses from liquid pathways assumed to be near zero. 
POPULATION DOSE-COMMITMENT ESTIMATES AND

AVERAGE INDIVIDUAL DOSE-COMMITMENT HISTOGRAM FOR

$$
\text { DRESDEN 1, } 2 \text { AND } 3
$$

Dose Commitments (person-rem) from Liquid Pathways

$\underline{\text { Cotal Body GI-LLI Thyroid Bone Liver }}$

[ No liquid pathways, thus doses essentially zero ]

Dose Commitments (person-rem) from Airborne Pathways Total Body GI-LLI Thyroid Bone Liver Lung

$\begin{array}{lllllll}\text { Infant } & 2.5 E+00 & 2.5 E+00 & 4.5 E+00 & 2.5 E+00 & 2.5 E+00 & 2.6 E+00 \\ \text { Child } & 2.7 E+01 & 2.7 E+01 & 4.7 E+01 & 2.9 E+01 & 2.8 E+01 & 2.9 E+01 \\ \text { Teen } & 2.0 E+01 & 2.0 E+01 & 3.0 E+01 & 2.1 E+01 & 2.0 E+01 & 2.2 E+01 \\ \text { Adult } & 1.2 E+02 & 1.2 E+02 & 1.6 E+02 & 1.2 E+02 & 1.2 E+02 & 1.3 E+02 \\ \text { TOTAL } & 1.7 E+02 & 1.7 E+02 & 2.5 E+02 & 1.8 E+02 & 1.7 E+02 & 1.8 E+02\end{array}$

Production/Consumption factors:

Produce: $<1 \quad$ Milk: <1 Meat: <1

FRACTION OF POPULATION RECEIVING AN INDICATED AVERAGE TOTAL-BODY DOSE COMMITMENT FROM AIRBORNE PATHWAYS

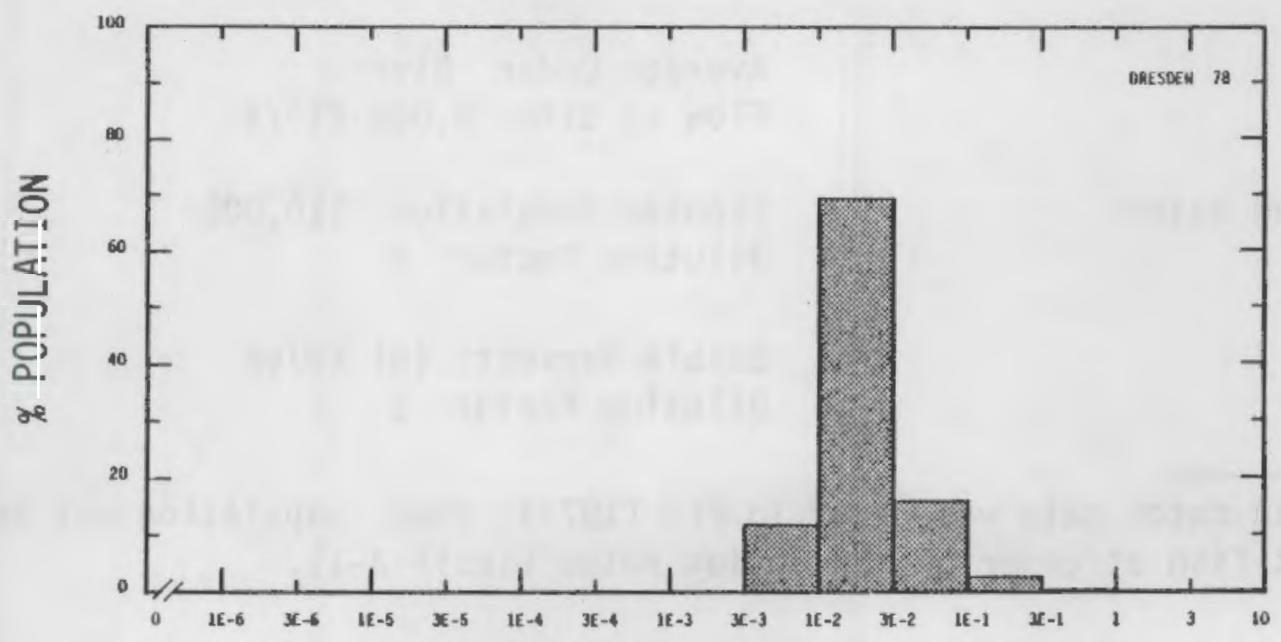

AVG INDIVIDUAL DOSE COMMITMENT - MILLIREM 


Location: N $42.09810 \quad$ W 91.77250

POPULATION DATA

Total Population Within 20-to-80-km Region: 5.7E5

Major Metropolitan Centers Within Region:

\begin{tabular}{lrrrr}
\multicolumn{1}{c}{ Center } & Population & \multicolumn{2}{c}{ Location } \\
\cline { 2 - 4 } Cedar Rapids & 110,000 & & \\
Waterlo0 & 77,000 & $66 \mathrm{~km}$ & SE \\
Iowa City & 48,000 & $63 \mathrm{~km}$ & NW \\
& & $53 \mathrm{~km}$ & SSE
\end{tabular}

SITE SPECIFIC DATA - AIRBORNE PATHWAYS

Average Annual State Production

of Crops and Animal Products

In $80-k m$ Radius Circle

Regional Productivity Factor:

Animal Grazing Factor:
Veg: $9.8 \mathrm{E} 7 \mathrm{kilogram}$

Milk: 2.6E 8 liter

Meat: $4.2 \mathrm{E} 8 \mathrm{kilogram}$

1

0.5

Meteorology Period of Record: 1 FEB 71 - 31 DEC 75 Recovery: 57\%

SITE SPECIFIC DATA - WATERBORNE PATHWAYS

Average Cedar River

Flow at Site: $3,000 \mathrm{ft} 3 / \mathrm{s}$

Drinking Water:

Exposed Population: 110,000

Dilution Factor: 1

Fish:

Edible Harvest: (a) $\mathrm{kg} / \mathrm{yr}$

Dilution Factor: 1

(a) No fish catch data was given in FES (1973); thus, population was assumed to eat fish at generic consumption rates (Table A-1). 
POPULATION DOSE-COMMITMENT ESTIMATES AND

AVERAGE INDIVIDUAL DOSE-COMMITMENT HISTOGRAM FOR

DUANE ARNOLD

Dose Commitments (person-rem) from Liquid Pathways

Total Body GI-LLI Thyroid Bone

$\begin{array}{llllll}\text { Infant } & 4.3 \mathrm{E}-03 & 3.8 \mathrm{E}-03 & 4.7 \mathrm{E}-03 & 2.6 \mathrm{E}-03 & 3.8 \mathrm{E}-03 \\ \text { Child } & 8.0 \mathrm{E}-02 & 1.2 \mathrm{E}-01 & 5.2 \mathrm{E}-02 & 1.4 \mathrm{E}-01 & 1.2 \mathrm{E}-01 \\ \text { Teen } & 5.8 \mathrm{E}-02 & 1.9 \mathrm{E}-01 & 2.1 \mathrm{E}-02 & 8.6 \mathrm{E}-02 & 8.3 \mathrm{E}-02 \\ \text { Adult } & 5.4 \mathrm{E}-01 & 1.6 \mathrm{E}+00 & 1.7 \mathrm{E}-01 & 5.9 \mathrm{E}-01 & 5.4 \mathrm{E}-01 \\ \text { TOTAL } & 6.8 \mathrm{E}-01 & 1.9 \mathrm{E}+00 & 2.5 \mathrm{E}-01 & 8.1 \mathrm{E}-01 & 7.4 \mathrm{E}-01\end{array}$

Dose Commitments (person-rem) from Airborne Pathways

Total Body GI-LLI Thyroid Bone Liver Lung

$\begin{array}{lllllll}\text { Infant } & 2.7 \mathrm{E}-03 & 2.7 \mathrm{E}-03 & 3.6 \mathrm{E}-02 & 2.8 \mathrm{E}-03 & 3.0 \mathrm{E}-03 & 2.7 \mathrm{E}-03 \\ \text { Child } & 3.1 \mathrm{E}-02 & 3.1 \mathrm{E}-02 & 4.5 \mathrm{E}-01 & 3.1 \mathrm{E}-02 & 3.4 \mathrm{E}-02 & 3.0 \mathrm{E}-02 \\ \text { Teen } & 2.3 \mathrm{E}-02 & 2.4 \mathrm{E}-02 & 2.5 \mathrm{E}-01 & 2.2 \mathrm{E}-02 & 2.5 \mathrm{E}-02 & 2.3 \mathrm{E}-02 \\ \text { Aduit } & 1.3 \mathrm{E}-01 & 1.4 \mathrm{E}-01 & 1.1 \mathrm{E}+00 & 1.3 \mathrm{E}-01 & 1.4 \mathrm{E}-01 & 1.3 \mathrm{E}-01 \\ \text { TOTAL } & 1.9 \mathrm{E}-01 & 2.0 \mathrm{E}-01 & 1.9 \mathrm{E}+00 & 1.9 \mathrm{E}-01 & 2.1 \mathrm{E}-01 & 1.9 \mathrm{E}-01\end{array}$

Production/Consumption factors:

Produce: <1 Milk: $3.4 \quad$ Meat: 9.1

FRACTION OF POPULATION RECEIVING AN INDICATED AVERAGE TOTAL-BODY DOSE COMMITMENT FROM AIRBORNE PATHWAYS

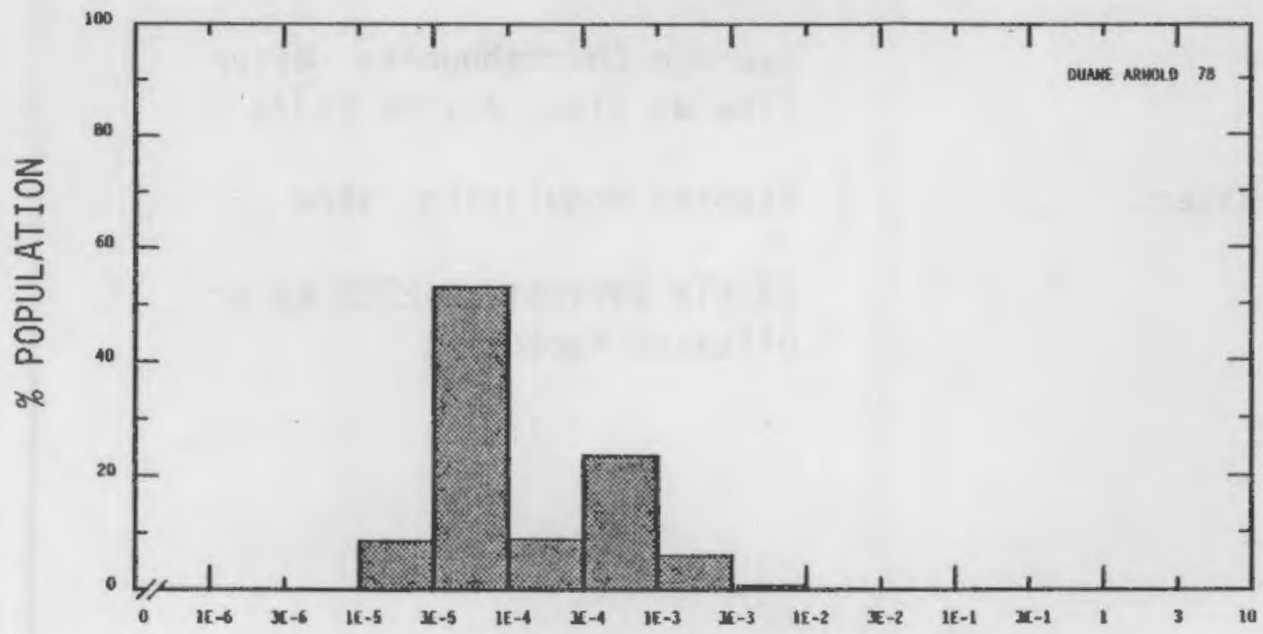

AVG INDIVIDUAL DOSE COMMITMENT - MILLIREM 
Location: N 31.22330 W 85.39330

POPULATION DATA

Total Population Within 20-to-80-km Region: 3.3E5

Major Metropolitan Centers Within Region:

\begin{tabular}{lccc}
\multicolumn{1}{c}{ Center } & Population & \multicolumn{2}{c}{ Location } \\
\cline { 2 - 3 } & 40,000 & & \\
Dothan & 17,000 & $27 \mathrm{~km}$ & W \\
Enterprise & 15,000 & $72 \mathrm{~km}$ & W \\
Ozark & & $60 \mathrm{~km}$ & NW
\end{tabular}

SITE SPECIFIC DATA - AIRBORNE PATHWAYS

Average Annual State Production

of Crops and Animal Products

In $80-\mathrm{km}$ Radius Circle

Regional Productivity Factor:

Animal Grazing Factor:
Veg: $1.7 \mathrm{E} 7 \mathrm{kilogram}$

Milk: 5.7E7 liter

Meat: 8.6E7 kilogram

0.95

0.8

Meteorology Period of Record: 1 APR 71 - 31 MAR 75 Recovery: 100\%

SITE SPECIFIC DATA - WATERBORNE PATHWAYS

Average Chattahoochee River

F1 ow at Site: $12,000 \mathrm{ft}^{3} / \mathrm{s}$

Orinking Water:

Exposed Population: None

Fish:

Edible Harvest: $2.27 \mathrm{E} 5 \mathrm{~kg} / \mathrm{yr}$

Dilution Factor: 1 
POPULATION DOSE-COMMITMENT ESTIMATES AND

AVERAGE INDIVIDUAL DOSE-COMMITMENT HISTOGRAM FOR

$$
\text { J. M. FARLEY }
$$

Dose Commitments (person-rem) from Liquid Pathways

Total Body GI-LLI $\underline{\text { Thyroid }}$ Bone Liver

Infant

Child

Teen

Adult

TOTAL

Infant

Child

Teen

Adult

TOTAL

$$
0.0 E+00
$$

4. $4 \mathrm{E}-04$

7. $9 \mathrm{E}-04$

8. 5E-03

9.7E-03
$0.0 \mathrm{E}+00$

2. $8 \mathrm{E}-04$

5. $8 \mathrm{E}-04$

5.1E-03

5. $9 \mathrm{E}-03$
$0.0 \mathrm{E}+00$

3.7E-05

2. $9 \mathrm{E}-05$

2.1E-04

2. $8 \mathrm{E}-04$

$0.0 \mathrm{E}+00$

$0.0 \mathrm{E}+00$

2. $5 \mathrm{E}-03$

2.1E-03

1. $2 \mathrm{E}-02$

1.7E-02

Dose Commitments (person-rem) from Airborne Pathways

Total Body GI-LLI Thyroid Bone Liver Lung

$\begin{array}{llllll}2.5 \mathrm{E}-03 & 2.5 \mathrm{E}-03 & 2.5 \mathrm{E}-03 & 2.3 \mathrm{E}-03 & 2.5 \mathrm{E}-03 & 2.6 \mathrm{E}-03 \\ 2.9 \mathrm{E}-02 & 2.9 \mathrm{E}-02 & 2.9 \mathrm{E}-02 & 2.5 \mathrm{E}-02 & 2.9 \mathrm{E}-02 & 3.1 \mathrm{E}-02 \\ 2.1 \mathrm{E}-02 & 2.1 \mathrm{E}-02 & 2.1 \mathrm{E}-02 & 1.8 \mathrm{E}-02 & 2.1 \mathrm{E}-02 & 2.3 \mathrm{E}-02 \\ 1.3 \mathrm{E}-01 & 1.3 \mathrm{E}-01 & 1.3 \mathrm{E}-01 & 1.1 \mathrm{E}-01 & 1.3 \mathrm{E}-01 & 1.3 \mathrm{E}-01 \\ 1.8 \mathrm{E}-01 & 1.8 \mathrm{E}-01 & 1.8 \mathrm{E}-01 & 1.6 \mathrm{E}-01 & 1.8 \mathrm{E}-01 & 1.9 \mathrm{E}-01\end{array}$

Production/Consumption factors:
Produce: $<1$
Milk: 1.3
Meat: 3.1

FRACTION OF POPULATION RECEIVING AN INDICATED AVERAGE TOTAL-BODY DOSE COMMITMENT FROM AIRBORNE PATHWAYS

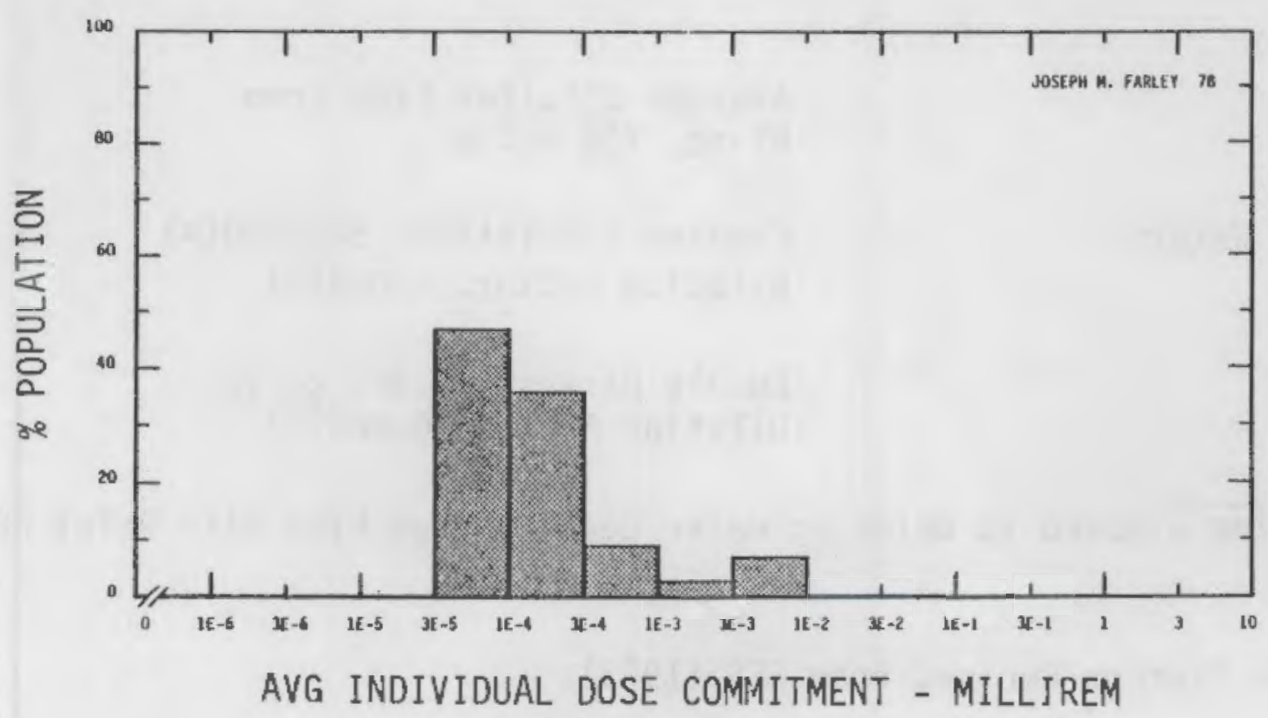


Site: J. A. FITZPATRICK OSWEGO, NEW YORK

Location: N $43.5217^{\circ}$ W $76.3980^{\circ}$

POPULATION DATA

Total Population Within 20-to-80-km Region: 8.255

Major Metropolitan Centers Within Region:

\begin{tabular}{|c|c|c|c|}
\hline Center & Population & \multicolumn{2}{|c|}{ Location } \\
\hline Syracuse SMSA & 620,000 & $54 \mathrm{~km}$ & SSE \\
\hline $\begin{array}{l}\text { Rome } \\
\text { Auburn }\end{array}$ & $\begin{array}{l}49,000 \\
34,000\end{array}$ & $\begin{array}{ll}80 & \mathrm{~km} \\
66 & \mathrm{~km}\end{array}$ & $\begin{array}{l}\text { ESE } \\
\text { SSW }\end{array}$ \\
\hline
\end{tabular}

SITE SPECIFIC DATA - AIRBORNE PATHWAYS

Average Annual State Production

of Crops and Animal Products

In $80-\mathrm{km}$ Radius Circle

Regional Productivity Factor:

Animal Grazing Factor:
Veg: $7.6 \mathrm{E} 7 \mathrm{kilogram}$

Milk: 7.0E8 liter

Meat: $3.3 E 7$ kilogram

0.7

0.5

Meteorology Period of Record: 1 JAN 74 - 31 DEC 75 Recovery: 97\%

SITE SPECIFIC DATA - WATERBORNE PATHWAYS

Average Dilution Flow from

Plant: $708 \mathrm{ft}^{3} / \mathrm{s}$

Drinking Water:

Exposed Population: $540,000(a)$

Dilution Factor: $0.003(b)$

Fish:

Edible Harvest: $7.3 E 5 \mathrm{~kg} / \mathrm{yr}$

Dilution Factor: 0.005 (b)

(a) Population exposed to drinking water derived from Nine Mile Point FES (1974).

(b) Dilution factors derived from FES (1973). 


Location: N 41.52000 W 96.07800

POPULATION DATA

Total Population Within 20-to-80-km Region: $7.5 \mathrm{E} 5$

$$
\begin{aligned}
& \text { Major Metropolitan Centers Within Region: } \\
& \text { Center } \quad \text { Population Location }
\end{aligned}
$$

$\begin{array}{lll}\text { Omaha SMSA } & 570,000 & 32 \mathrm{~km}\end{array}$

SITE SPECIFIC DATA - AIRBORNE PATHWAYS

Average Annual State Production

of Crops and Animal Products

In 80-km Radius Circle

Regional Productivity Factor:

Animal Grazing Factor:

Meteorology Period of Record: 1 JAN 74 - 31 DEC 74 Recovery: 98\%
Veg: $9.7 E 7$ kilogram Milk: 7.2E7 liter Meat: 2.0E8 kilogram

1

0.5

SITE SPECIFIC DATA - WATERBORNE PATHWAYS

Average Missouri River Flow at Site: $27,000 \mathrm{ft}^{3} / \mathrm{s}$

Drinking Water: Exposed Population: $570,000(a)$ Dilution Factor: 1

Fish: Edible Harvest: $1.0 E 4 \mathrm{~kg} / \mathrm{yr}$ Dilution Factor: 1

(a) Drinking water pollution assumed to be Omaha SMSA (FES, 1972). 
POPULATION DOSE-COMMITMENT ESTIMATES AND

AVERAGE INDIVIOUAL DOSE-COMMITMENT HISTOGRAM FOR

J. A. FITZPATRICK

Dose Commitments (person-rem) from Liquid Pathways

Total Body GI-LLI Ihyroid Bone Liver

Infant

Child

Teen

Adult

TOTAL

2. $1 \mathrm{E}-04$

1.6E-04

2.8E-03

$5.2 \mathrm{E}-04$

8.6E-04

1.1E-02

4. $2 \mathrm{E}-03$

2.0E-02

4.1E-02

5.2E-02

1.7E-02

5.6E-03

6. $3 \mathrm{E}-03$

2. $3 \mathrm{E}-02$

4.1E-02

1. $8 \mathrm{E}-01$

5.2E-02

4. $4 \mathrm{E}-02$

1. $3 \mathrm{E}-01$

2.5E-01

2.1E-01

6. $2 \mathrm{E}-02$

$7.3 E-02$

2. $0 \mathrm{E}-01$

3. $4 \mathrm{E}-01$

Dose Commitments (person-rem) from Airborne Pathways

Total Body GI-LLI Thyroid Bone Liver Lung

$\begin{array}{lllllll}\text { Infant } & 2.4 \mathrm{E}-03 & 2.3 \mathrm{E}-03 & 1.3 \mathrm{E}-01 & 2.7 \mathrm{E}-03 & 2.9 \mathrm{E}-03 & 2.4 \mathrm{E}-03 \\ \text { Child } & 2.7 \mathrm{E}-02 & 2.5 \mathrm{E}-02 & 8.5 \mathrm{E}-01 & 3.0 \mathrm{E}-02 & 3.0 \mathrm{E}-02 & 2.7 \mathrm{E}-02 \\ \text { Teen } & 1.9 \mathrm{E}-02 & 1.9 \mathrm{E}-02 & 3.2 \mathrm{E}-01 & 2.0 \mathrm{E}-02 & 2.0 \mathrm{E}-02 & 2.0 \mathrm{E}-02 \\ \text { Adult } & 1.1 \mathrm{E}-01 & 1.1 \mathrm{E}-01 & 1.1 \mathrm{E}+00 & 1.1 \mathrm{E}-01 & 1.2 \mathrm{E}-01 & 1.2 \mathrm{E}-01 \\ \text { TOTAL } & 1.6 \mathrm{E}-01 & 1.6 \mathrm{E}-01 & 2.4 \mathrm{E}+00 & 1.7 \mathrm{E}-01 & 1.7 \mathrm{E}-01 & 1.7 \mathrm{E}-01\end{array}$

Production/Consumption factors:
Produce: $<1$
Milk: 4.6
Meat: <1

FRACTION OF POPULATION RECEIVING AN INOICATEO AVERAGE TOTAL-BODY DOSE COMMITMENT FROM AIRBORNE PATHWAYS

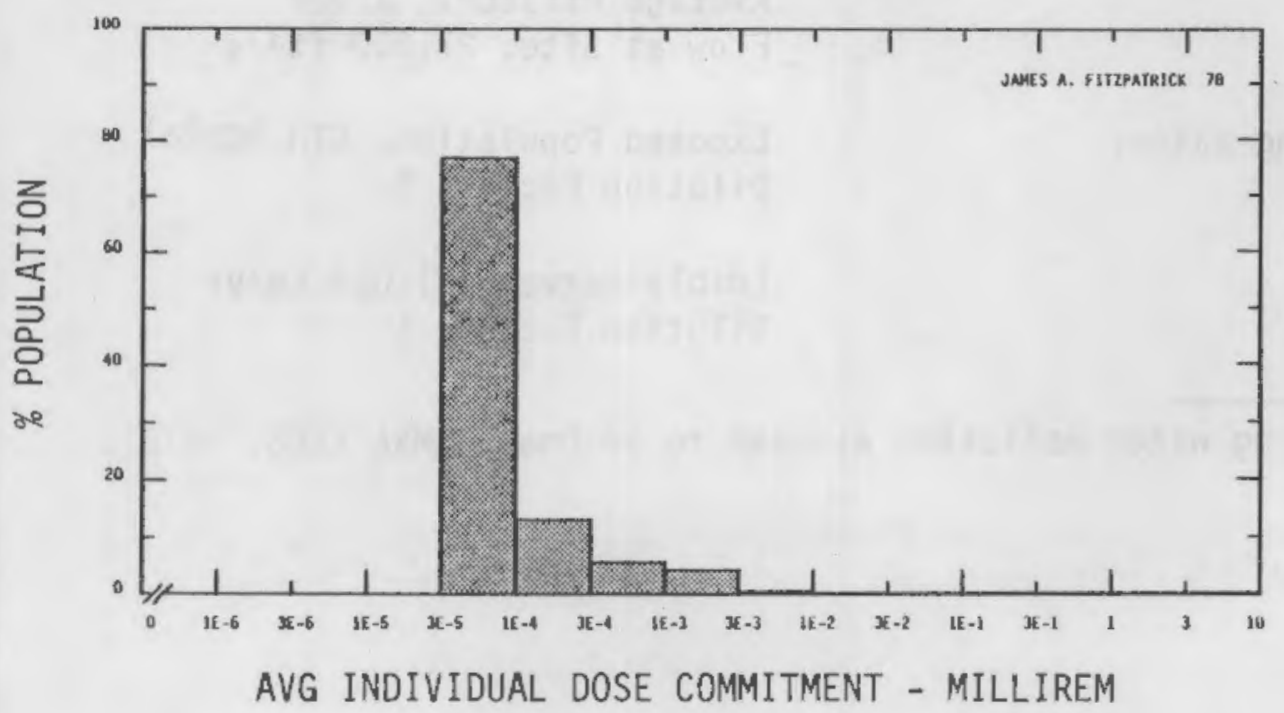


POPULATION DOSE-COMMITMENT ESTIMATES AND

AVERAGE INOIVIDUAL DOSE-COMMITMENT HISTOGRAM FOR FORT CALHOUN

Dose Commitments (person-rem) from Liquid Pathways

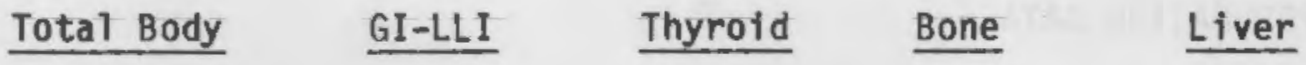

Infant

Child

Teen

Adult

TOTAL

4.2E-03

2. $9 \mathrm{E}-03$

3. $5 \mathrm{E}-02$

$1.1 \mathrm{E}-02$

1. $8 \mathrm{E}-02$

5. $8 \mathrm{E}-02$

3. $3 \mathrm{E}-02$

2. 5E-01

1. 3E-01

1. $8 \mathrm{E}-01$

3. $5 \mathrm{E}-02$

1. $4 \mathrm{E}-02$

8.0E-02

3. $5 \mathrm{E}-02$

6.7E-02

3. $6 \mathrm{E}-01$

1.2E-01

5.8E-01

2.2E-01

4. 5E-01

4. $6 \mathrm{E}-01$

1.7E-01

9.5E -01

3. $9 \mathrm{E}-01$

7.1E-01

Dose Commitments (person-rem) from Airborne Pathways

\section{Total Body GI-LLI Thyroid Bone Liver Lung}

Infant

Child

Teen

Adult

TOTAL
2.2E-04

2.5E-03

$1.8 \mathrm{E}-03$

$1.1 \mathrm{E}-02$

2.2E- -04

2.5E-03

2.5E-04

$1.8 \mathrm{E}-03$

1.1E-02

1.5E-02

1.5E-02
2. $9 \mathrm{E}-03$

2. $0 \mathrm{E}-03$

$1.2 \mathrm{E}-02$

2.1E-04

2. $3 \mathrm{E}-03$

$1.7 E-03$

$1.0 \mathrm{E}-02$

1.7 $\mathrm{E}-02$

1.5E-02

2.2E-04

2.3E-04

2.5E -03

2.7E-03

$1.8 \mathrm{E}-03$

2.1E -03

1.2E-02

$1.5 \mathrm{E}-02$

1.7E-02

Production/Consumption factors:

Produce: <1

Milk: <1

Meat: 3.3

FRACTION OF POPULATION RECEIVING AN INDICATED AVERAGE TOTAL-BODY DOSE COMMITMENT FROM AIRBORNE PATHWAYS

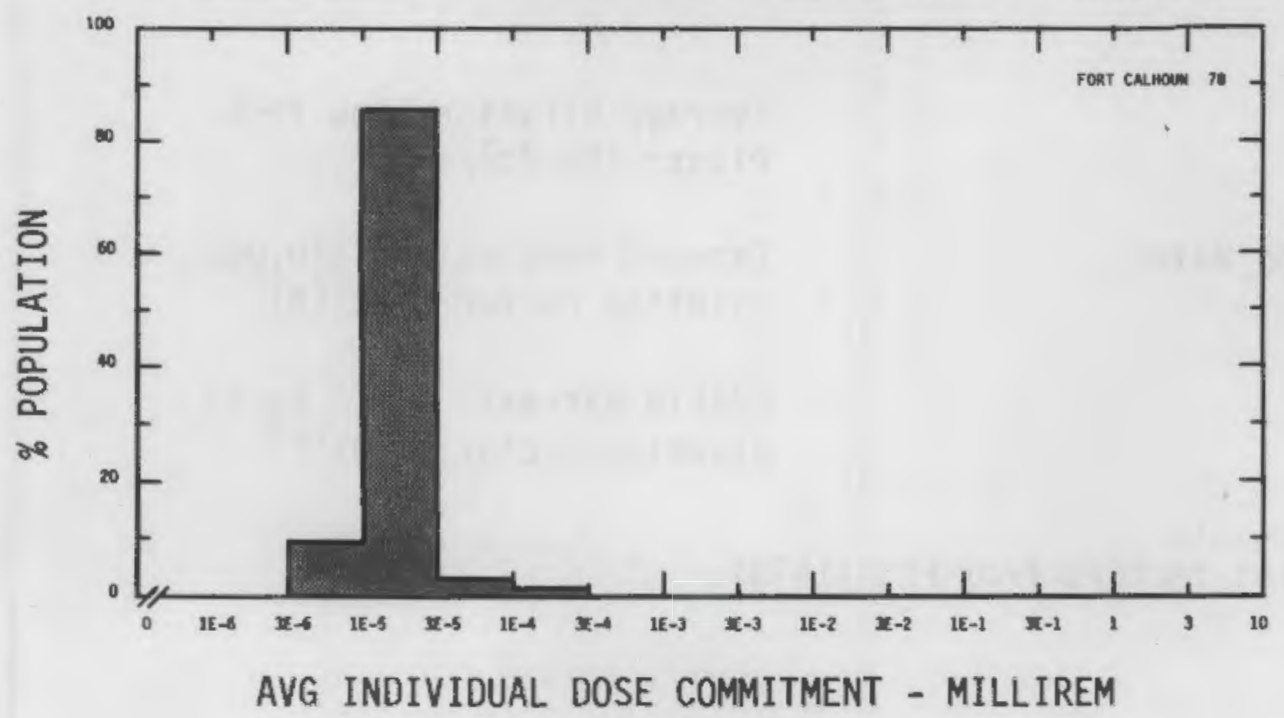




Location: N 43.27720 W 77.30810

POPULATION DATA

Total Population within 20-to-80-km Region: 1.1E6

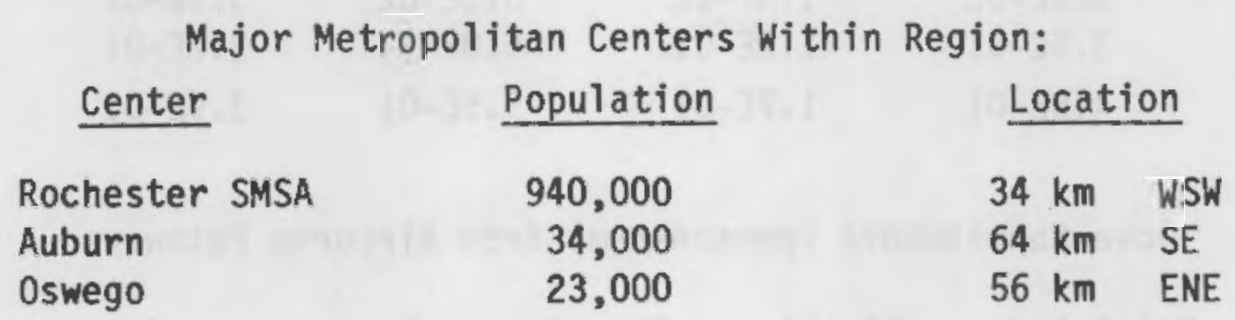

SITE SPECIFIC DATA - AIRBORNE PATHWAYS

Average Annual State Production

of Crops and Animal Products

In 80-km Radius Circle

Regional Productivity Factor:

Animal Grazing Factor:
Veg: $7.6 \mathrm{E} 7 \mathrm{kilogram}$ Milk: $7.0 \mathrm{E} 8$ liter Meat: $3.3 E 7$ kilogram

0.6

0.5

Meteorology Period of Record: 1 JAN 66 - 31 DEC 67 Recovery: $89 \%$

Average Dilution Flow from Plant: $753 \mathrm{ft}^{3} / \mathrm{s}$

Drinking Water:

Fish:
Exposed Population: 540,000 Dilution Factor: 0.01 (a)

Edible Harvest: $7.3 \mathrm{E} 5 \mathrm{~kg} / \mathrm{yr}$ Dilution Factor: 0.01 (a)

(a) Dilution factors from FES (1973). 

AVERAGE INDIVIDUAL DOSE-COMMITMENT HISTOGRAM FOR

$$
\text { R. E. GINNA }
$$

Dose Commitments (person-rem) from Liquid Pathways

Total Body GI-LLI Thyroid Bone

$\begin{array}{llllll}\text { Infant } & 1.5 \mathrm{E}-05 & 1.5 \mathrm{E}-03 & 4.3 \mathrm{E}-03 & 2.0 \mathrm{E}-04 & 1.7 \mathrm{E}-03 \\ \text { Child } & 1.8 \mathrm{E}-02 & 1.7 \mathrm{E}-02 & 3.7 \mathrm{E}-02 & 9.8 \mathrm{E}-03 & 2.7 \mathrm{E}-02 \\ \text { Teen } & 9.2 \mathrm{E}-03 & 7.2 \mathrm{E}-03 & 1.3 \mathrm{E}-02 & 5.1 \mathrm{E}-03 & 1.4 \mathrm{E}-02 \\ \text { Adult } & 8.5 \mathrm{E}-02 & 6.2 \mathrm{E}-02 & 9.8 \mathrm{E}-02 & 3.0 \mathrm{E}-02 & 9.9 \mathrm{E}-02 \\ \text { TOTAL } & 1.1 \mathrm{E}-01 & 8.7 \mathrm{E}-02 & 1.5 \mathrm{E}-01 & 4.5 \mathrm{E}-02 & 1.4 \mathrm{E}-01\end{array}$

Dose Commitments (person-rem) from Airborne Pathways Total Body GI-LLI Thyroid Bone Liver Lung

$\begin{array}{lllllll}\text { Infant } & 3.2 \mathrm{E}-04 & 3.2 \mathrm{E}-04 & 3.3 \mathrm{E}-03 & 1.7 \mathrm{E}-04 & 3.3 \mathrm{E}-04 & 3.4 \mathrm{E}-04 \\ \text { Child } & 4.0 \mathrm{E}-03 & 3.9 \mathrm{E}-03 & 2.2 \mathrm{E}-02 & 1.8 \mathrm{E}-03 & 4.0 \mathrm{E}-03 & 4.2 \mathrm{E}-03 \\ \text { Teen } & 2.6 \mathrm{E}-03 & 2.6 \mathrm{E}-03 & 9.6 \mathrm{E}-03 & 1.3 \mathrm{E}-03 & 2.6 \mathrm{E}-03 & 3.0 \mathrm{E}-03 \\ \text { Adult } & 1.5 \mathrm{E}-02 & 1.5 \mathrm{E}-02 & 3.8 \mathrm{E}-02 & 7.8 \mathrm{E}-03 & 1.5 \mathrm{E}-02 & 1.6 \mathrm{E}-02 \\ \text { TOTAL } & 2.2 \mathrm{E}-02 & 2.2 \mathrm{E}-02 & 7.3 \mathrm{E}-02 & 1.1 \mathrm{E}-02 & 2.2 \mathrm{E}-02 & 2.3 \mathrm{E}-02\end{array}$

Production/Consumption factors:

Produce: $<1 \quad$ Milk: $2.8 \quad$ Meat: $<1$

FRACTION OF POPULATION RECEIVING AN INDICATED AVERAGE TOTAL-BODY OOSE COMMITMENT FROM AIRBORNE PATHWAYS

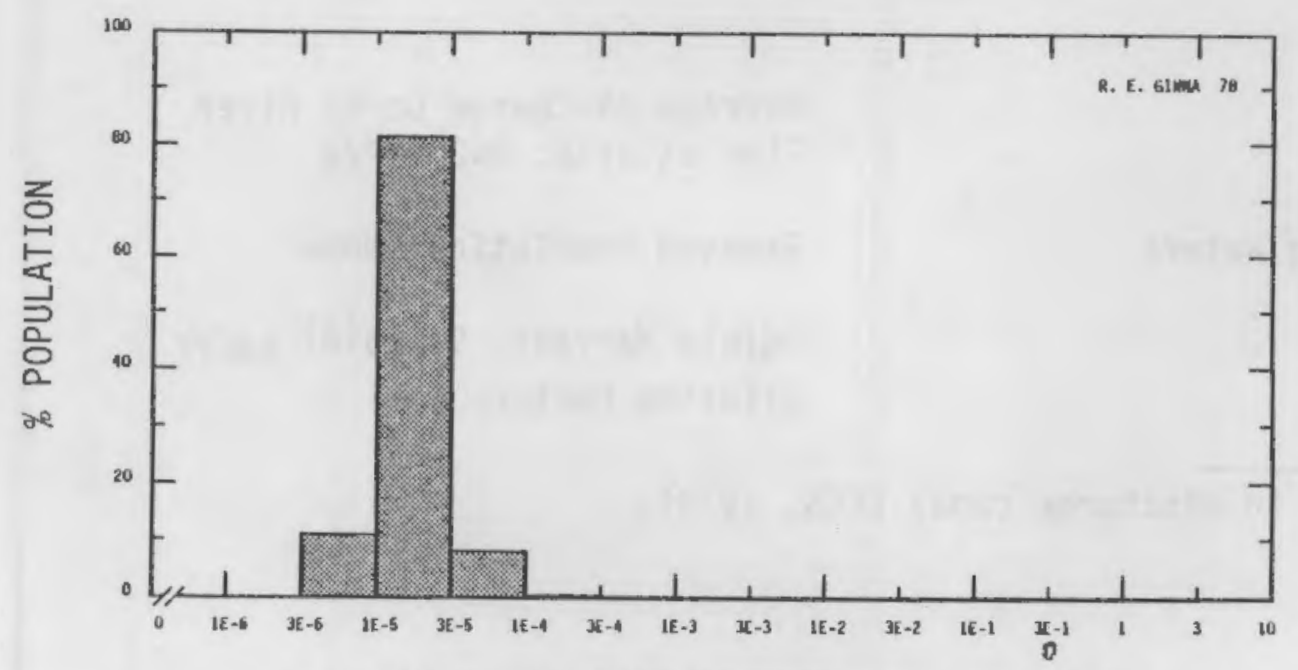

AVG INDIVIDUAL DOSE COMMITMENT - MILLIREM 
Site: HADDAM NECK (CONN. YANKEE) HADDAM NECK, CONNECTICUT

Location: N $41.4822^{\circ} \quad$ W 72.49890

POPULATION DATA

Total Population Within 20-to-80-km Region: $3.3 \mathrm{E} 6$

Major Metropolitan Centers Within Region:

Center

Hartford SMSA

New Haven SMSA

Springfield SMSA

Waterbury
Population

830,000

760,000

600,000

110,000
Location

$37 \mathrm{~km} \quad \mathrm{NW}$

$40 \mathrm{~km}$ WSW

$72 \mathrm{~km} \mathrm{~N}$

$47 \mathrm{~km} \mathrm{~W}$

SITE SPECIFIC DATA - AIRBORNE PATHWAYYS

Average Annual State Production

of Crops and Animal Products

In 80-km Radius Circle

Regional Productivity Factor:

Animal Grazing Factor:
Veg: $3.2 E 7$ kilogram

Milk: 4.4E8 1iter

Meat: $2.0 \mathrm{E} 7 \mathrm{kilogram}$

0.7

0.6

Meteorology Period of Record: 1 JAN 75 - 31 DEC 75 Recovery: 95\%

Average Discharge Canal River

Flow at Site: $862 \mathrm{ft}^{3} / \mathrm{s}$

Drinking Water:

Fish:
Exposed Population: None

Edible Harvest: $9.1 \mathrm{E3}(\mathrm{a}) \mathrm{kg} / \mathrm{yr}$

Dilution Factor: 1

(a) Caught in discharge canal (FES, 1973). 
POPULATION DOSE-COMMITMENT ESTIMATES AND

AVERAGE INDIVIDUAL DOSE-COMMITMENT HISTOGRAM FOR

HADDAM NECK

Dose Commitments (person-rem) from Liquid Pathways

Total Body

$\underline{\text { GI-LLI }}$

Thyroid

Bone

Liver

Infant

$0.0 E+00$

$0.0 \mathrm{E}+00$

$0.0 \mathrm{E}+00$

$0.0 E+00$

$0.0 \mathrm{E}+00$

Child

Teen

Adult

5.1E-02

2. $5 \mathrm{E}-03$

1. $8 \mathrm{E}-03$

2.3E-01

2. $8 \mathrm{E}-01$

9.9E-02

4. $3 \mathrm{E}-03$

1. $4 \mathrm{E}-03$

1. $4 \mathrm{E}-01$

2. $4 \mathrm{E}-01$

1. $1 \mathrm{E}+00$

3. $6 \mathrm{E}-02$

9. $7 \mathrm{E}-03$

8.1E-01

$1.4 \mathrm{E}+00$

TOTAL

1. $2 \mathrm{E}+00$

4.3E-02

1. $3 \mathrm{E}-02$

1. $2 \mathrm{E}+00$

2. $0 \mathrm{E}+00$

Dose Commitments (person-rem) from Airborne Pathways

Total Body GI-LLI Thyroid Bone Liver Lung

$\begin{array}{lllllll}\text { Infant } & 2.9 \mathrm{E}-01 & 2.9 \mathrm{E}-01 & 3.0 \mathrm{E}-01 & 1.3 \mathrm{E}+00 & 2.9 \mathrm{E}-01 & 2.9 \mathrm{E}-01 \\ \text { Child } & 1.8 \mathrm{E}+00 & 1.8 \mathrm{E}+00 & 1.9 \mathrm{E}+00 & 9.0 \mathrm{E}+00 & 1.8 \mathrm{E}+00 & 1.8 \mathrm{E}+00 \\ \text { Teen } & 5.8 \mathrm{E}-01 & 5.8 \mathrm{E}-01 & 5.9 \mathrm{E}-01 & 2.7 \mathrm{E}+00 & 5.8 \mathrm{E}-01 & 5.8 \mathrm{E}-01 \\ \text { Adult } & 1.7 \mathrm{E}+00 & 1.7 \mathrm{E}+00 & 1.8 \mathrm{E}+00 & 7.7 \mathrm{E}+00 & 1.7 \mathrm{E}+00 & 1.7 \mathrm{E}+00 \\ \text { TOTAL } & 4.4 \mathrm{E}+00 & 4.4 \mathrm{E}+00 & 4.5 \mathrm{E}+00 & 2.1 \mathrm{E}+01 & 4.4 \mathrm{E}+00 & 4.4 \mathrm{E}+00\end{array}$

Production/Consumption factors:

Produce: $<1$

Milk: $<1$

Meat: $<1$

FRACTION OF POPULATION RECEIVING AN INDICATED AVERAGE TOTAL-BODY DOSE COMMITMENT FROM AIRBORNE PATHWAYS

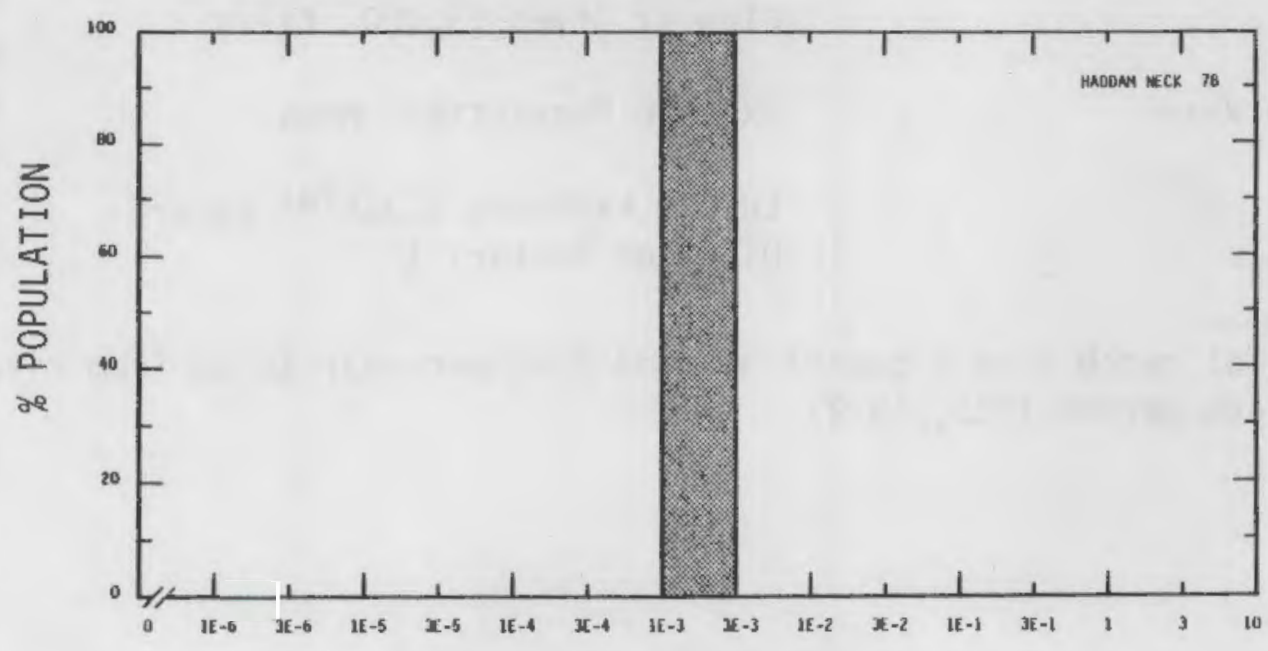

AVG INDIVIDUAL DOSE COMMITMENT - MILLIREM 
Location: N 31.93440 W $82.3444^{\circ}$

POPULATION DATA

Total Population Within 20-to-80-km Region: $2.8 \mathrm{E} 5$

Major Metropolitan Centers Within Region:

Center

Waycross
Population

21,000
Location

$80 \mathrm{~km} \mathrm{~S}$

SITE SPECIFIC DATA - AIRBORNE PATHWAYS

Average Annual State Production

of Crops and Animal Products

In 80-km Radius Circle

Regional Productivity Factor:

Animal Grazing Factor:
Veg: $8.8 \mathrm{E} 6 \mathrm{kilogram}$

Milk: 7.0E7 liter

Meat: $8.1 \mathrm{E} 7 \mathrm{kilogram}$

1

0.8

Meteorology Period of Record: 1 JUN 70 - 31 AUG 74 Recovery: 87\%

SITE SPECIFIC DATA - WATERBORNE PATHWAYS

Average Altamaha River

Flow at Site: $13,000 \mathrm{ft}^{3} / \mathrm{s}$

Drinking Water:

Exposed Population: None

Fish:

Edible Harvest: $6.3 E 5(\mathrm{a}) \mathrm{kg} / \mathrm{yr}$ Dilution Factor: 1

(a) Commercial catch plus 3 pounds of game fish per year taken from river by average person (FES, 1972). 
POPULATION DOSE-COMMITMENT ESTIMATES AND

AVERAGE INDIVIDUAL DOSE-COMMITMENT HISTOGRAM FOR

E. I. HATCH

Dose Commitments (person-rem) from Liquid Pathways

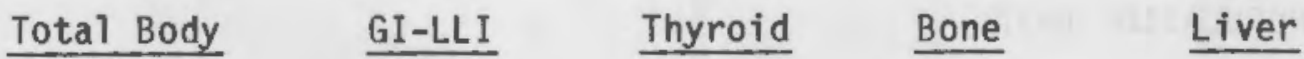

Infant

$0.0 \mathrm{E}+00$

$0.0 \mathrm{E}+00$

$0.0 E+00$

$0.0 \mathrm{E}+00$

$0.0 E+00$

Child

4. $3 \mathrm{E}-03$

9. $4 \mathrm{E}-04$

1.1E-04

1.5E-02

1.8E-02

Teen

$6.5 \mathrm{E}-03$

2. $0 \mathrm{E}-03$

8.1E-05

9.1E-03

$1.5 \mathrm{E}-02$

Adult

$6.5 \mathrm{E}-\mathrm{D} 2$

$1.8 \mathrm{E}-02$

$5.4 \mathrm{E}-04$

$5.4 \mathrm{E}-02$

9.2E-02

TOTAL

$7.6 \mathrm{E}-02$

$2.1 \mathrm{E}-02$

7. $3 \mathrm{E}-04$

$7.8 \mathrm{E}-02$

$1.2 \mathrm{E}-\mathrm{D} 1$

Dose Commitments (person-rem) from Airborne Pathways Total Body GI-LLI Thyroid Bone Liver Lung

$\begin{array}{lllllll}\text { Infant } & 1.5 \mathrm{E}-04 & 1.4 \mathrm{E}-04 & 5.8 \mathrm{E}-04 & 1.7 \mathrm{E}-04 & 1.9 \mathrm{E}-04 & 1.6 \mathrm{E}-04 \\ \text { Child } & 1.7 \mathrm{E}-03 & 1.6 \mathrm{E}-03 & 4.0 \mathrm{E}-03 & 1.9 \mathrm{E}-03 & 2.0 \mathrm{E}-03 & 1.8 \mathrm{E}-03 \\ \text { Teen } & 1.2 \mathrm{E}-03 & 1.2 \mathrm{E}-03 & 2.1 \mathrm{E}-03 & 1.2 \mathrm{E}-03 & 1.3 \mathrm{E}-03 & 1.4 \mathrm{E}-03 \\ \text { Adu1t } & 7.5 \mathrm{E}-03 & 7.1 \mathrm{E}-03 & 1.0 \mathrm{E}-02 & 7.1 \mathrm{E}-03 & 7.6 \mathrm{E}-03 & 7.7 \mathrm{E}-03 \\ \text { TOTAL. } & 1.1 \mathrm{E}-02 & 1.0 \mathrm{E}-02 & 1.7 \mathrm{E}-02 & 1.0 \mathrm{E}-02 & 1.1 \mathrm{E}-02 & 1.1 \mathrm{E}-02\end{array}$

Production/Consumption factors:

Produce: <1 Milk: 1.9 Meat: 3.6

FRACTION OF POPULATION RECEIVING AN INDICATED AVERAGE TOTAL-BODY DOSE COMMITMENT FROM AIRBORNE PATHWAYS

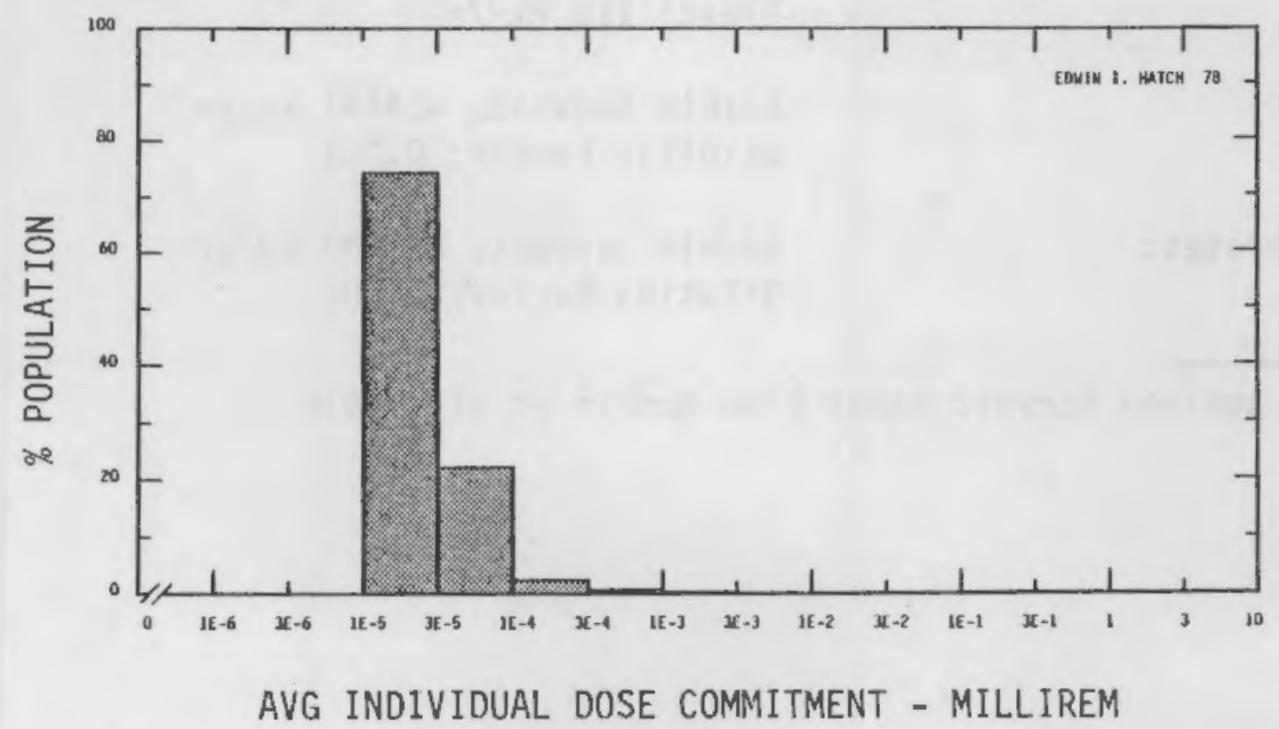


Location: N $40.7417^{\circ} \quad$ W $124.2081^{\circ}$

POPULATION DATA

Total Population Within 20-to-80-km Region: $1.1 \mathrm{E5}$

Major Metropolitan Centers Within Region:

\begin{tabular}{lcc} 
Center & Population & \multicolumn{1}{l}{ Location } \\
\cline { 2 - 3 } & 27,000 & $6.4 \mathrm{~km} \mathrm{~N}$
\end{tabular}

SITE SPECIFIC DATA - AIRBORNE PATHWAYS

Average Annual State Production

of Crops and Animal Products

In $80-\mathrm{km}$ Radius Circle

Regional Productivity Factor:

Animal Grazing Factor:
Veg: $4.8 E 7$ kilogram

Milk: 2.3E8 liter

Meat: 5.0E7 kilogram

0.5

1

Meteorology Period of Record: 1 JAN 66 - 31 DEC 67 Recovery: 96\%

SITE SPECIFIC DATA - WATERBORNE PATHWAYS

Average Dilution Flow from

Plant: $112 \mathrm{ft}^{3} / \mathrm{s}$

Fish:

Edible Harvest: $4 E 4(\mathrm{a}) \mathrm{kg} / \mathrm{yr}$

Dilution Factor: 0.001

Invertebrates:

Edible Harvest: $5 \mathrm{E} 3(\mathrm{a}) \mathrm{kg} / \mathrm{yr}$

Dilution Factor: 0.002

(a) Edible seafood harvest taken from Noskin et a1., 1976. 
AVERAGE INDIVIDUAL DOSE-COMMITMENT HISTOGRAM FOR

HUMBOLDT BAY

Dose Commitments (person-rem) from Liquid Pathways

Total Body GI-LLI $\underline{\text { Thyroid }}$ Bone Liver

$\begin{array}{llllll}\text { Infant } & 0.0 \mathrm{E}+00 & 0.0 \mathrm{E}+00 & 0.0 \mathrm{E}+00 & 0.0 \mathrm{E}+00 & 0.0 \mathrm{E}+00 \\ \text { Child } & 1.3 \mathrm{E}-05 & 1.6 \mathrm{E}-05 & 2.0 \mathrm{E}-10 & 4.4 \mathrm{E}-05 & 4.9 \mathrm{E}-05 \\ \text { Teen } & 1.8 \mathrm{E}-05 & 3.2 \mathrm{E}-05 & 1.8 \mathrm{E}-10 & 2.6 \mathrm{E}-05 & 4.1 \mathrm{E}-05 \\ \text { Adult } & 1.8 \mathrm{E}-04 & 2.8 \mathrm{E}-04 & 1.4 \mathrm{E}-09 & 1.5 \mathrm{E}-04 & 2.4 \mathrm{E}-04 \\ \text { TOTAL } & 2.1 \mathrm{E}-04 & 3.2 \mathrm{E}-04 & 1.8 \mathrm{E}-09 & 2.2 \mathrm{E}-04 & 3.3 \mathrm{E}-04\end{array}$

Dose Commitments (person-rem) from Airborne Pathways

Total Body GI-LLI Thyroid Bone Liver Lung

$\begin{array}{lllllll}\text { Infant } & 2.0 \mathrm{E}-06 & 1.9 \mathrm{E}-06 & 1.8 \mathrm{E}-06 & 3.9 \mathrm{E}-06 & 2.3 \mathrm{E}-06 & 1.8 \mathrm{E}-05 \\ \text { Child } & 2.7 \mathrm{E}-05 & 2.5 \mathrm{E}-05 & 2.0 \mathrm{E}-05 & 7.8 \mathrm{E}-05 & 2.8 \mathrm{E}-05 & 3.0 \mathrm{E}-04 \\ \text { Teen } & 2.0 \mathrm{E}-05 & 2.4 \mathrm{E}-05 & 1.5 \mathrm{E}-05 & 5.6 \mathrm{E}-05 & 2.0 \mathrm{E}-05 & 2.7 \mathrm{E}-04 \\ \text { Adult } & 1.2 \mathrm{E}-04 & 1.5 \mathrm{E}-04 & 9.0 \mathrm{E}-05 & 3.1 \mathrm{E}-04 & 1.1 \mathrm{E}-04 & 1.1 \mathrm{E}-03 \\ \text { TOTAL } & 1.7 \mathrm{E}-04 & 2.0 \mathrm{E}-04 & 1.3 \mathrm{E}-04 & 4.5 \mathrm{E}-04 & 1.6 \mathrm{E}-04 & 1.7 \mathrm{E}-03\end{array}$

Production/Consumption factors:
Produce: 1.1
Milk: 7.9
Meat: 2.8

FRACTION OF POPULATION RECEIVING AN INDICATED AVERAGE TOTAL-BODY DOSE COMMITMENT FROM AIRBORNE PATHWAYS

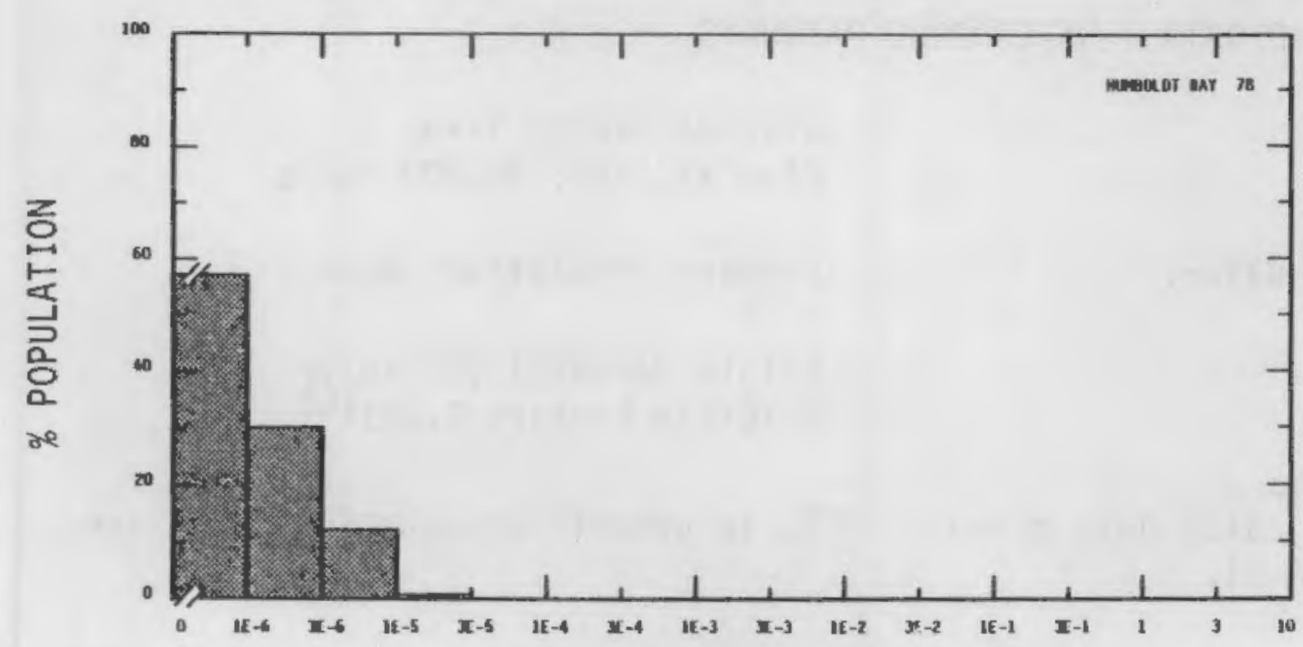

AVG INDIVIDUAL DOSE COMMITMENT - MILLI?EM 
Site: INDIAN POINT

BUCHANAN, NEW YORK

Location: N $41.2656^{\circ}$ W $73.9350^{\circ}$

POPILATION DATA

Total Population Within 20-to-80-km Region: 1.6E7

Major Metropolitan Centers Within Region:

Center

New York SMSA

Newark SMSA

Stamford

Norwalk

Bridgeport

Poughkeepsie

White Plains
Population

$9,700,000$

$2,000,000$

110,000

77,000

150,000

31,000

49,000

\section{Location}

$60 \mathrm{~km} \quad \mathrm{~s}$

$64 \mathrm{~km}$ SSW

$42 \mathrm{~km} \quad \mathrm{SE}$

$48 \mathrm{~km}$ ESE

$64 \mathrm{~km} \quad \mathrm{E}$

$48 \mathrm{~km} \mathrm{~N}$

$30 \mathrm{~km}$ SSE

SITE SPECIFIC DATA - AIRBORNE PATHWAYS

Average Annual State Production

of Crops and Animal Products

In 80-km Radius Circle

Regional Productivity Factor:

Animal Grazing Factor:
Veg: $7.6 \mathrm{E} 7 \mathrm{kilogram}$

Milk: 7.0E8 liter

Meat: $3.3 \mathrm{E} 7 \mathrm{kilogram}$

0.8

0.5

Meteorology Period of Record: 1 JAN 75 - 31 DEC 75 Recovery: 96\%

SITE SPECIFIC DATA - WATERBORNE PATHWAYS

Average Hudson River

Flow at Site: $20,000 \mathrm{ft}^{3} / \mathrm{s}$

Drinking Water:

Exposed Population: None

Fish:

Edible Harvest: (a) $\mathrm{kg} / \mathrm{yr}$

Dilution Factor: $0.001(b)$

(a) No fish catch data given in FES, so generic consumption rates used (Table A-1).

(b) One percent of population obtain $10 \%$ of their fish from river (FES, 1972). 
POPULATION DOSE-COMMITMENT ESTIMATES ANO

AVERAGE INDIVIOUAL DOSE-COMMITMENT HISTOGRAM FOR

INDIAN POINT 1,2 ANO 3

Dose Commitments (person-rem) from Liquid Pathways

Total Body GI-LLI $\underline{\text { Thyroid }}$ Bone Liver

$\begin{array}{llllll}\text { Infant } & 0.0 \mathrm{E}+00 & 0.0 \mathrm{E}+00 & 0.0 \mathrm{E}+00 & 0.0 \mathrm{E}+00 & 0.0 \mathrm{E}+00 \\ \text { Child } & 3.7 \mathrm{E}-02 & 3.3 \mathrm{E}-03 & 3.8 \mathrm{E}-03 & 1.9 \mathrm{E}-01 & 2.1 \mathrm{E}-01 \\ \text { Teen } & 7.0 \mathrm{E}-02 & 6.7 \mathrm{E}-03 & 2.8 \mathrm{E}-03 & 1.1 \mathrm{E}-01 & 1.8 \mathrm{E}-01 \\ \text { Adult } & 7.6 \mathrm{E}-01 & 5.7 \mathrm{E}-02 & 1.8 \mathrm{E}-02 & 6.6 \mathrm{E}-01 & 1.1 \mathrm{E}+00 \\ \text { TOTAL } & 8.7 \mathrm{E}-01 & 6.7 \mathrm{E}-02 & 2.5 \mathrm{E}-02 & 9.6 \mathrm{E}-01 & 1.5 \mathrm{E}+00\end{array}$

Dose Commi tments (person-rem) from Airborne Pathways

Total Body GI-LLI Thyroid Bone Liver Lung

$\begin{array}{lllllll}\text { Infant } & 1.1 \mathrm{E}-01 & 1.0 \mathrm{E}-01 & 1.6 \mathrm{E}-01 & 1.3 \mathrm{E}-01 & 1.3 \mathrm{E}-01 & 1.2 \mathrm{E}-01 \\ \text { Chi1d } & 1.2 \mathrm{E}+00 & 1.2 \mathrm{E}+00 & 1.6 \mathrm{E}+00 & 1.3 \mathrm{E}+00 & 1.4 \mathrm{E}+00 & 1.3 \mathrm{E}+00 \\ \text { Teen } & 8.8 \mathrm{E}-01 & 8.4 \mathrm{E}-01 & 1.1 \mathrm{E}+00 & 9.0 \mathrm{E}-01 & 9.5 \mathrm{E}-01 & 1.0 \mathrm{E}+00 \\ \text { Adult } & 5.3 \mathrm{E}+00 & 5.1 \mathrm{E}+00 & 6.0 \mathrm{E}+00 & 5.3 \mathrm{E}+00 & 5.4 \mathrm{E}+00 & 5.7 \mathrm{E}+00 \\ \text { TOTAL } & 7.5 \mathrm{E}+00 & 7.2 \mathrm{E}+00 & 8.8 \mathrm{E}+00 & 7.6 \mathrm{E}+00 & 7.9 \mathrm{E}+00 & 8.2 \mathrm{E}+00\end{array}$

Production/Consumption factors:

Produce: $<1 \quad$ Milk: $<1 \quad$ Meat: $<1$

FRACTION OF POPULATION RECEIVING AN INDICATED AVERAGE TOTAL-BODY DOSE COMMITMENT FROM AIRBORME PATHWAYS

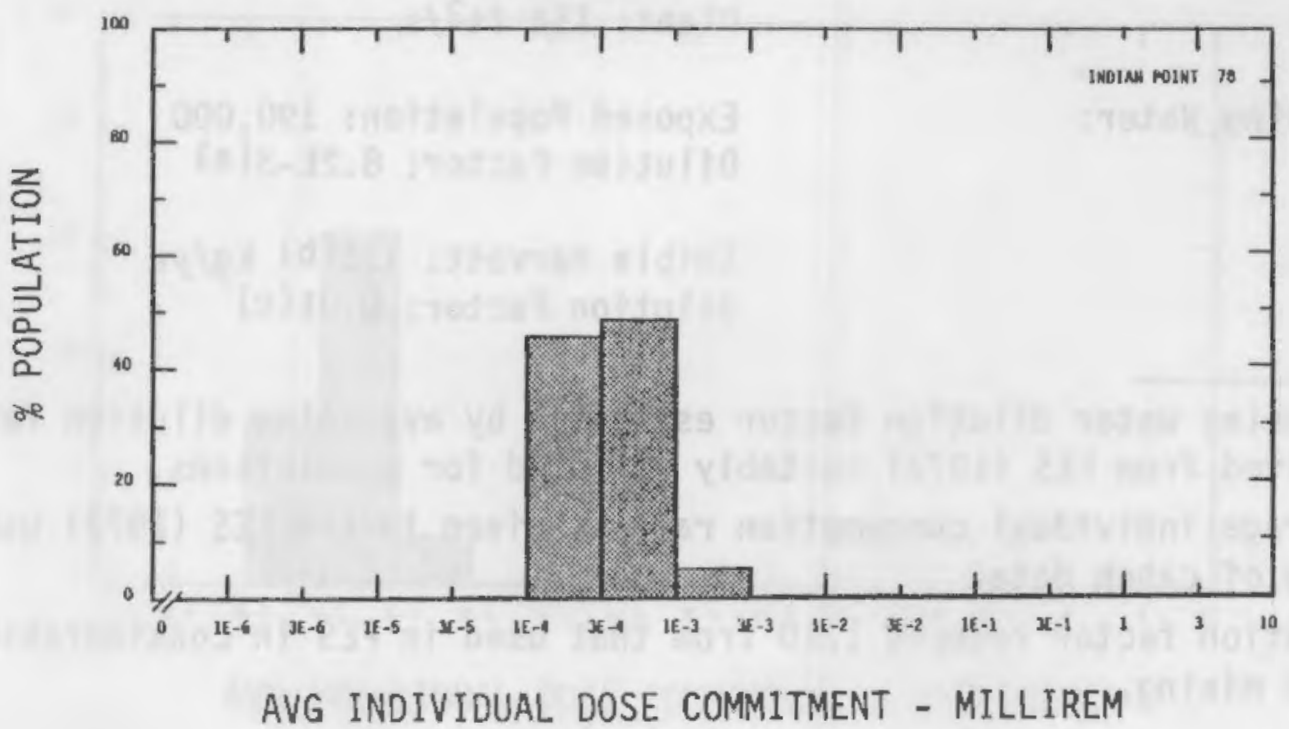




Location: N 44.34330 W 87.53500

POPULATION DATA

Total Population Within 20-to-80-km Region: 6.0E5

Major Metropolitan Centers Within Region:

\begin{tabular}{lcccc} 
Center & Population & & \multicolumn{2}{c}{ Location } \\
\cline { 2 - 6 } & & & \\
Sheboygan & 51,000 & $64 \mathrm{~km}$ & SSW \\
Manitowoc & 35,000 & $30 \mathrm{~km}$ & SSW \\
Greenbay & 93,000 & $40 \mathrm{~km}$ & W \\
Appleton & 60,000 & $72 \mathrm{~km}$ & WSW
\end{tabular}

SITE SPECIFIC DATA - AIRBORNE PATHWAYS

Average Annual State Production

of Crops and Animal Products

In 80-km Radius Circle

Regional Productivity Factor:

Animal Grazing Factor:
Veg: $7.2 E 7 \mathrm{kilogram}$

Milk: 1.2Eg liter

Meat: $1.0 \mathrm{E} 8 \mathrm{kilogram}$

0.5

0.5

Meteorology Period of Record: 2 JAN 69 - 31 DEC 69 Recovery: 76\%

SITE SPECIFIC DATA - WATERBORNE PATHWAYS

Average Dilution Flow from Plant: $154 \mathrm{ft}^{3} / \mathrm{s}$

Drinking Water:

Exposed Population: 190,000

Dilution Factor: $8.2 E-3(a)$

Fish:

Edible Harvest: $1.1(\mathrm{~b}) \mathrm{kg} / \mathrm{yr}$ Dilution Factor: $0.01(c)$

(a) Drinking water dilution factor estimated by averaging dilution factors derived from FES (1972) suitably weighted for populations.

(b) Average individual consumption rate as given in the FES (1972) used in lieu of catch data.

(c) Dilution factor reduced $1 / 10$ from that used in FES in consideration of Take mixing. 
POPULATION DOSE-COMMITMENT ESTIMATES AND AVERAGE INDIVIDUAL DOSE-COMMITMENT HISTOGRAM FOR

\section{KEWAUNEE}

Dose Commitments (person-rem) from Liquid Pathways

Total Body GI-LLI Thyroid Bone Liver

Infant

2.9E-03

2.9E-03

$8.8 \mathrm{E}-03$

7.1E-04

3. $6 \mathrm{E}-03$

Child

Teen

Adult

5.1E-02

4.2E-02

7.5E-02

8.7E-02

1.4E-01

4.7E-02

3.1E-02

2. $6 \mathrm{E}-02$

5. $0 \mathrm{E}-02$

9. $8 \mathrm{E}-02$

4. $8 \mathrm{E}-01$

2.7E-01

2. $0 \mathrm{E}-01$

2. $9 \mathrm{E}-01$

6.1E-01

TOTAL

$5.8 \mathrm{E}-01$

3. 5E-01

3.1E-01

4. 3E-01

8.5E-01

Dose Commitments (person-rem) from Airborne Pathways Total Body GI-LLI Thyroid Bone Liver Lung

Infant

1.3E-04 1.3E-04

Child

1. $8 \mathrm{E}-03$

1. $6 \mathrm{E}-03$

$6.2 \mathrm{E}-04$

$1.0 \mathrm{E}-04$

1. $3 \mathrm{E}-04$

1. 4E-04

Teen

1. $2 \mathrm{E}-03$

1.1E-03

5. $3 \mathrm{E}-03$

1. $8 \mathrm{E}-03$

1. $6 \mathrm{E}-03$

1. $8 \mathrm{E}-03$

Adult

6.7E-03

6. $4 \mathrm{E}-03$

2. $8 \mathrm{E}-03$

1.1E-03

1.1E-03

1. $3 \mathrm{E}-03$

TOTAL

9.7 $\mathrm{E}-03$

9. $3 \mathrm{E}-03$

1. $3 \mathrm{E}-02$

6. $4 \mathrm{E}-03$

6. $4 \mathrm{E}-03$

7. $0 \mathrm{E}-03$

2.2E-02

9. $4 \mathrm{E}-03$

9. $3 \mathrm{E}-03$

1. $0 \mathrm{E}-02$

Production/Consumption factors:

Produce: $<1$

Milk: 7.4

Meat: 1.1

FRACTION OF POPULATION RECEIVING AN INDICATED AVERAGE TOTAL-BODY DOSE COMMITMENT FROM AIRBORNE PATHWAYS

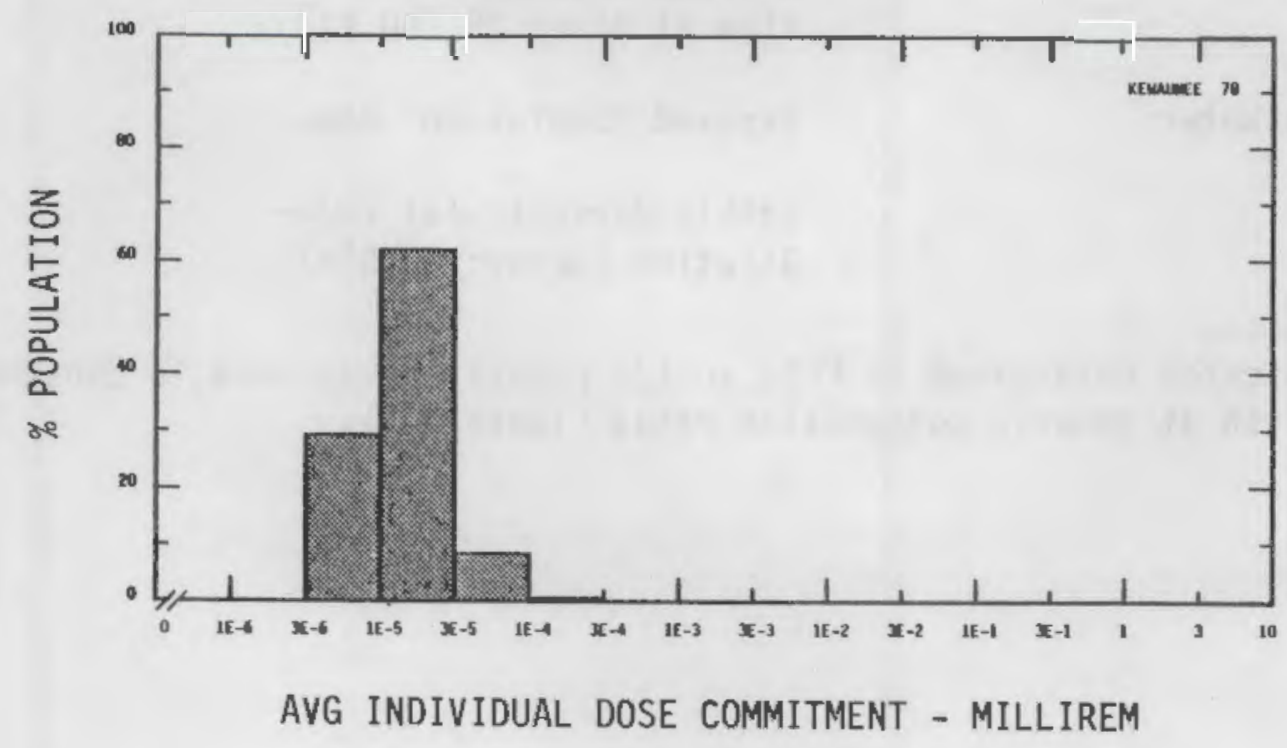


Location: N 43.55970 W $91.2281^{\circ}$

POPULATION DATA

Total Population Within 20-to-80-km Region: 3.3E5

Major Metropolitan Centers Within Region:

$\begin{array}{lll}\frac{\text { Center }}{\text { La Crosse }} & \text { Population } & \text { Location } \\ 54,000 & 32 \mathrm{~km} \mathrm{~N}\end{array}$

SITE SPECIFIC DATA - AIRBORNE PATHWAYS

Average Annual State Production

of Crops and Animal Products

In 8D-km Radius Circle

Regional Productivity Factor:

Animal Grazing Factor:
Veg: 7.2E7 kilogram

Milk: 1.2E9 liter

Meat: $1.0 \mathrm{E} 8$ kilogram

1

0.5

Meteorology Period of Record: 1 JAN 75 - 31 DEC 75 Recovery: 97\%

SITE SPECIFIC DATA - WATERBORNE PATHWAYS

Average Mississippi River

Flow at site: $28,000 \mathrm{ft}^{3} / \mathrm{s}$

Drinking Water:

Exposed Population: None

Fish:

Edible Harvest: (a) $\mathrm{kg} / \mathrm{yr}$

Dilution Factor: $0.5(\mathrm{a})$

(a) No fish catch data given in FES, so $1 / 2$ population assumed to consume river fish at generic consumption rates (Table A-1). 
POPULATION DOSE-COMMITMENT ESTIMATES AND AVERAGE INDIVIDUAL DOSE-COMMITMENT HISTOGRAM FOR

\section{LA CROSSE}

Dose Commitments (person-rem) from Liquid Pathways

Total Body

GI-LLI

Thyroid

Bone

Liver

Infant

$0.0 \mathrm{E}+00$

$0.0 E+00$

$0.0 \mathrm{E}+00$

$0.0 E+00$

$0.0 E+00$

Child

2.5E-01

3. $0 \mathrm{E}-01$

2.2E-02

1. $3 \mathrm{E}+00$

1. $3 E+00$

Teen

Adult.

4.5E-01

6.2E-01

1. $6 \mathrm{E}-02$

7. $8 \mathrm{E}-01$

$1.1 E+00$

TOTAL

4. $8 \mathrm{E}+00$

$5.4 E+00$

1. $0 \mathrm{E}-01$

$4.5 \mathrm{E}+00$

$6.8 \mathrm{E}+00$

$5.5 E+00$

6. $3 \mathrm{E}+00$

$1.4 E-01$

$6.6 \mathrm{E}+00$

$9.2 E+00$

Dose Commitments (person-rem) from Airborne Pathways

Total Body GI-LLI Thyroid Bone Liver Lung

$\begin{array}{lllllll}\text { Infant } & 5.3 \mathrm{E}-03 & 5.3 \mathrm{E}-03 & 9.3 \mathrm{E}-03 & 5.3 \mathrm{E}-03 & 5.3 \mathrm{E}-03 & 5.5 \mathrm{E}-03 \\ \text { Child } & 6.0 \mathrm{E}-02 & 5.9 \mathrm{E}-02 & 1.0 \mathrm{E}-01 & 6.0 \mathrm{E}-02 & 5.9 \mathrm{E}-02 & 6.2 \mathrm{E}-02 \\ \text { Teen } & 4.3 \mathrm{E}-02 & 4.3 \mathrm{E}-02 & 6.0 \mathrm{E}-02 & 4.4 \mathrm{E}-02 & 4.3 \mathrm{E}-02 & 4.6 \mathrm{E}-02 \\ \text { Adult } & 2.6 \mathrm{E}-01 & 2.6 \mathrm{E}-01 & 3.3 \mathrm{E}-01 & 2.6 \mathrm{E}-01 & 2.6 \mathrm{E}-01 & 2.7 \mathrm{E}-01 \\ \text { TOTAL } & 3.7 \mathrm{E}-01 & 3.7 \mathrm{E}-01 & 5.0 \mathrm{E}-01 & 3.7 \mathrm{E}-01 & 3.7 \mathrm{E}-00 & 3.9 \mathrm{E}-01\end{array}$

Production/Consumption factors:
Produce: 1.1
Milk: 27
Meat: 3.8

FRACTION OF POPULATION RECEIVING AN INDICATED AVERAGE TDTAL-BODY DOSE COMMITMENT FROM AIRBORNE PATHWAYS

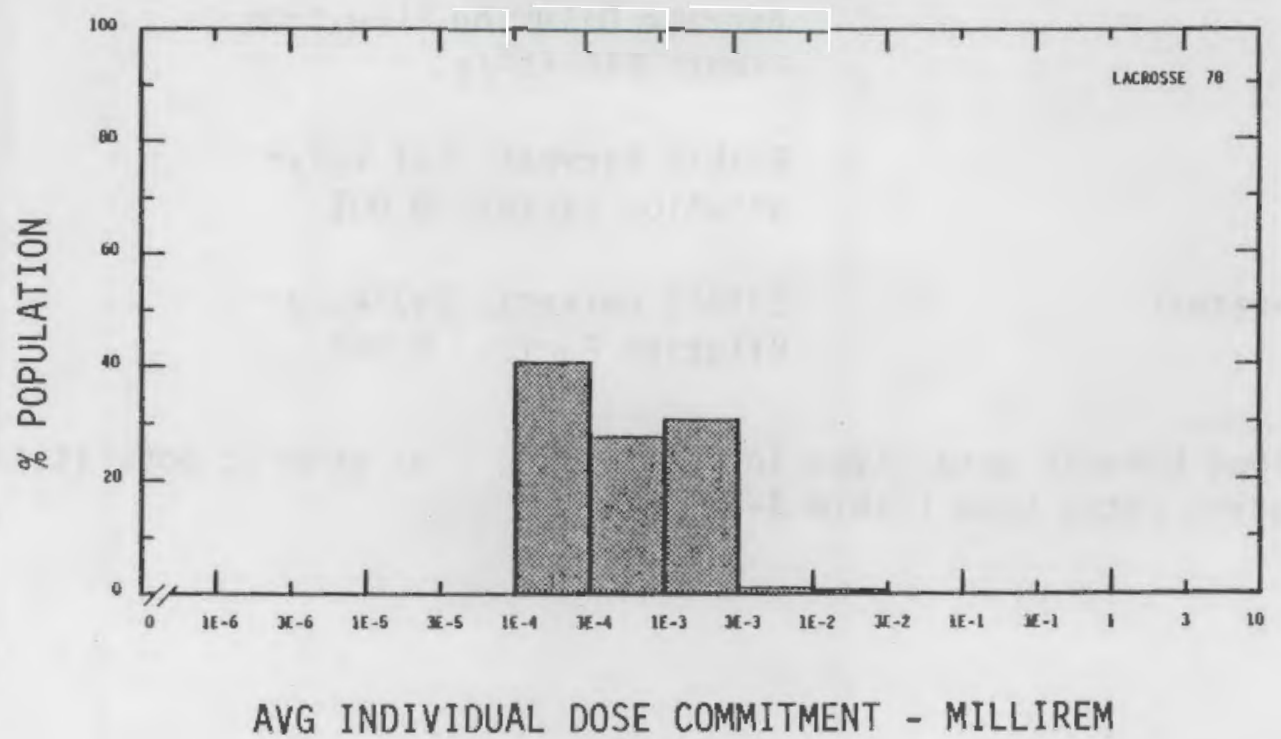




Location: $\quad$ N $43.95030 \quad$ W 69.69640

POPULATION DATA

Total Population Within 20-to-80-km Region: 5.7E5

Major Metropolitan Centers Within Region:

\begin{tabular}{lcccc} 
Center & Population & & \multicolumn{2}{c}{ Location } \\
\cline { 2 - 4 } & 71,000 & $51 \mathrm{~km}$ & $\mathrm{SW}$ \\
Portland & 46,000 & $40 \mathrm{~km}$ & $\mathrm{NW}$ \\
Lewiston & & &
\end{tabular}

SITE SPECIFIC DATA - AIRBORNE PATHWAYS

Average Annual State Production

of Crops and Animal Products

In $80-\mathrm{km}$ Radius Circle

Regional Productivity Factor:

Animal Grazing Factor:

Meteorology Period of Record: 1 APR 75 - 31 MAR 76 Recovery: 98\%
Veg: 2.4E8 kilogram

Milk: $6.6 \mathrm{E} 7$ liter

Meat: $4.3 \mathrm{E} 6 \mathrm{kilogram}$

0.6

0.5

SITE SPECIFIC DATA - WATERBORNE PATHWAYS

Average Dilution Flow from P1 ant: $844 \mathrm{ft}^{3} / \mathrm{s}$

Fish:

Edible Harvest: (a) $\mathrm{kg} / \mathrm{yr}$

Dilution Factor: 0.001

Invertebrates:

Edible Harvest: (a) $\mathrm{kg} / \mathrm{yr}$

Dilution Factor: 0.002

(a) No seafood harvest data given in FES (1972), thus generic population consumption rates used (Table A-1). 


\section{POPULATION DOSE-COMMITMENT ESTIMATES ANO}

AVERAGE INOIVIOUAL OOSE-COMMITMENT HISTOGRAM FOR

\section{MAINE YANKEE}

Dose Commitments (person-rem) from Liquid Pathways

Total Body GI-LLI Thyroid Bone Liver

$\begin{array}{lllllr}\text { Infant } & 0.0 \mathrm{E}+00 & 0.0 \mathrm{E}+00 & 0.0 \mathrm{E}+00 & 0.0 \mathrm{E}+00 & 0.0 \mathrm{E}+00 \\ \text { Child } & 3.6 \mathrm{E}-05 & 3.6 \mathrm{E}-05 & 1.6 \mathrm{E}-03 & 4.1 \mathrm{E}-05 & 5.6 \mathrm{E}-05 \\ \text { Teen } & 3.5 \mathrm{E}-05 & 5.2 \mathrm{E}-05 & 1.1 \mathrm{E}-03 & 2.4 \mathrm{E}-05 & 4.8 \mathrm{E}-05 \\ \text { Adult } & 2.9 \mathrm{E}-04 & 4.5 \mathrm{E}-04 & 7.3 \mathrm{E}-03 & 1.4 \mathrm{E}-04 & 3.2 \mathrm{E}-04 \\ \text { TOTAL } & 3.6 \mathrm{E}-04 & 5.3 \mathrm{E}-04 & 9.9 \mathrm{E}-03 & 2.1 \mathrm{E}-04 & 4.3 \mathrm{E}-04\end{array}$

Dose Commitments (person-rem) from Airborne Pathways

Total Body GI-LLI Thyroid Bone Liver Lung

$\begin{array}{lllllll}\text { Infant } & 3.6 \mathrm{E}-04 & 3.6 \mathrm{E}-04 & 6.6 \mathrm{E}-04 & 3.6 \mathrm{E}-04 & 3.6 \mathrm{E}-04 & 4.0 \mathrm{E}-04 \\ \text { Child } & 4.2 \mathrm{E}-03 & 4.2 \mathrm{E}-03 & 8.5 \mathrm{E}-03 & 4.0 \mathrm{E}-03 & 4.2 \mathrm{E}-03 & 4.7 \mathrm{E}-03 \\ \text { Teen } & 3.0 \mathrm{E}-03 & 3.0 \mathrm{E}-03 & 4.8 \mathrm{E}-03 & 2.9 \mathrm{E}-03 & 3.0 \mathrm{E}-03 & 3.7 \mathrm{E}-03 \\ \text { Adult } & 1.8 \mathrm{E}-02 & 1.8 \mathrm{E}-02 & 2.5 \mathrm{E}-02 & 1.8 \mathrm{E}-02 & 1.8 \mathrm{E}-02 & 2.0 \mathrm{E}-02 \\ \text { TOTAL } & 2.6 \mathrm{E}-02 & 2.6 \mathrm{E}-02 & 3.9 \mathrm{E}-02 & 2.5 \mathrm{E}-02 & 2.6 \mathrm{E}-02 & 2.9 \mathrm{E}-02\end{array}$

Production/Consumption factors:

Produce: 1.3 Milk: <1 Meat: <1

FRACTION OF POPULATION RECEIVING AN INDICATED AVERAGE TOTAL-BODY DOSE COMMITMENT FROM AIRBORNE PATHWAYS

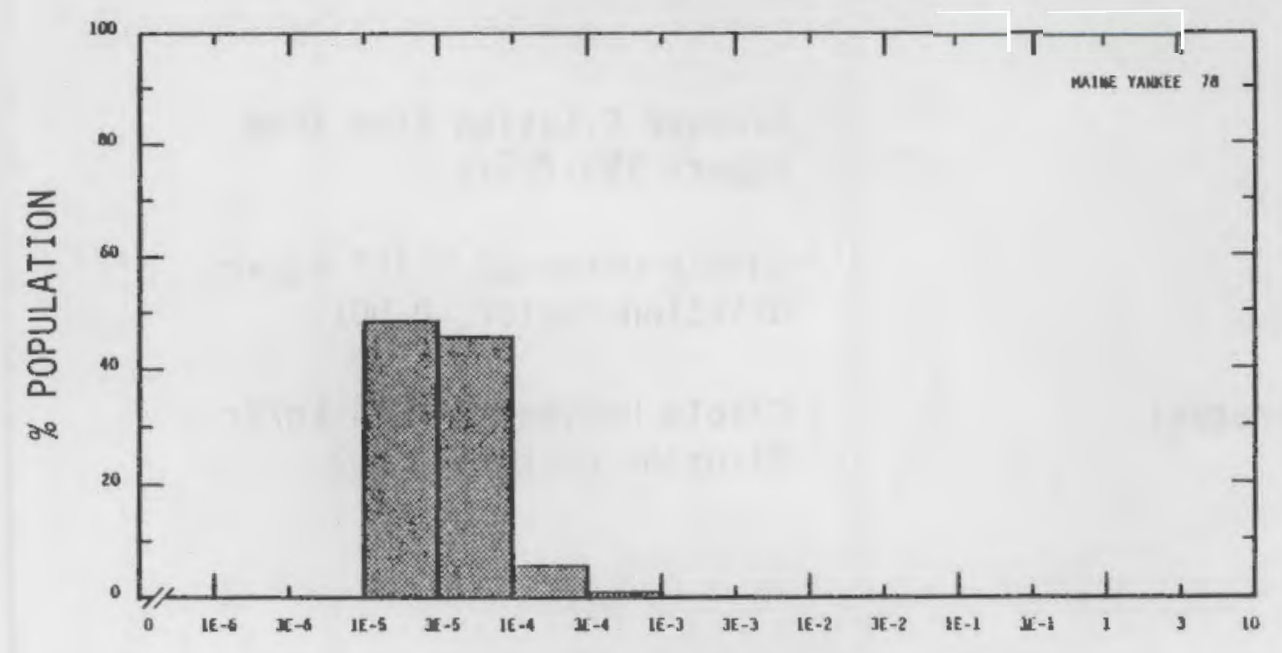

AVG INDIVIDUAL DOSE COMMITMENT - MILLIREM 
Site: MILLSTONE POINT

WATERFORD, CONNECTICUT

Location: $\quad$ N $41.30860 \quad$ W 72.16750

POPULATION DATA

Total Population Within 20-to-80-km Region: 2.5E6

Major Metropolitan Centers Within Region:

Center Population Location

$\begin{array}{lrrl}\text { Hartford SMSA } & 830,000 & 72 \mathrm{~km} & \text { NW } \\ \text { New Haven SMSA } & 760,000 & 64 \mathrm{~km} & \text { W } \\ \text { Warwick } & 86,000 & 80 \mathrm{~km} & \text { NE } \\ \text { Waterbury } & 110,000 & 70 \mathrm{~km} & \text { WNW }\end{array}$

SITE SPECIFIC DATA - AIRBORNE PATHWAYS

Average Annual State Production

of Crops and Animal Products

In $80-\mathrm{km}$ Radius Circle

Regional Productivity Factor:

Animal Grazing Factor:
Veg: $3.2 E 7$ kilogram

Milk: $4.4 \mathrm{E} 8$ liter

Meat: $2.0 \mathrm{E} 7$ kilogram

0.6

0.6

Meteorology Period of Record: 1 JAN $74-31$ DEC 74 Recovery: 95\%

SITE SPECIFIC DATA - WATERBORNE PATHWAYS

Average Dilution Flow from

Plant: $135 \mathrm{ft}^{3} / \mathrm{s}$

Fish:

Edible Harvest: $9.1 \mathrm{E} 4 \mathrm{~kg} / \mathrm{yr}$

Dilution Factor: 0.001

Invertebrates:

Edible Harvest: $9.1 \mathrm{E} 4 \mathrm{~kg} / \mathrm{yr}$

Dilution Factor: 0.002 
POPULATION DOSE-COMMITMENT ESTIMATES AND AVERAGE INDIVIDUAL DOSE-COMMITMENT HISTOGRAM FOR

MILLSTONE POINT 1 AND 2

Dose Commitments (person-rem) from Liquid Pathways

Total Body GI-LLI $\underline{\text { Thyroid Bone Liver }}$

$\begin{array}{llllll}\text { Infant } & 0.0 \mathrm{E}+00 & 0.0 \mathrm{E}+00 & 0.0 \mathrm{E}+00 & 0.0 \mathrm{E}+00 & 0.0 \mathrm{E}+00 \\ \text { Child } & 1.2 \mathrm{E}-03 & 3.0 \mathrm{E}-03 & 1.5 \mathrm{E}-04 & 4.0 \mathrm{E}-04 & 8.8 \mathrm{E}-04 \\ \text { Teen } & 8.8 \mathrm{E}-04 & 6.3 \mathrm{E}-03 & 1.1 \mathrm{E}-04 & 2.4 \mathrm{E}-04 & 7.6 \mathrm{E}-04 \\ \text { Adult } & 6.0 \mathrm{E}-03 & 5.5 \mathrm{E}-02 & 7.0 \mathrm{E}-04 & 1.4 \mathrm{E}-03 & 4.6 \mathrm{E}-03 \\ \text { TOTAL } & 8.0 \mathrm{E}-03 & 6.5 \mathrm{E}-02 & 9.5 \mathrm{E}-04 & 2.0 \mathrm{E}-03 & 6.3 \mathrm{E}-03\end{array}$

Dose Commitments (person-rem) from Airborne Pathways

Total Body GI-LLI Thyroid Bone Liver Lung

$\begin{array}{lllllll}\text { Infant } & 2.9 \mathrm{E}+00 & 2.9 \mathrm{E}+00 & 7.4 \mathrm{E}+00 & 2.9 \mathrm{E}+00 & 2.9 \mathrm{E}+00 & 3.0 \mathrm{E}+00 \\ \text { Child } & 3.2 \mathrm{E}+01 & 3.2 \mathrm{E}+01 & 5.7 \mathrm{E}+01 & 3.2 \mathrm{E}+01 & 3.2 \mathrm{E}+01 & 3.3 \mathrm{E}+01 \\ \text { Teen } & 2.4 \mathrm{E}+01 & 2.4 \mathrm{E}+01 & 3.3 \mathrm{E}+01 & 2.4 \mathrm{E}+01 & 2.4 \mathrm{E}+01 & 2.5 \mathrm{E}+01 \\ \text { Adult } & 1.4 \mathrm{E}+02 & 1.4 \mathrm{E}+02 & 1.7 \mathrm{E}+02 & 1.4 \mathrm{E}+02 & 1.4 \mathrm{E}+02 & 1.5 \mathrm{E}+02 \\ \text { TOTAL } & 2.0 \mathrm{E}+02 & 2.0 \mathrm{E}+02 & 2.7 \mathrm{E}+02 & 2.0 \mathrm{E}+02 & 2.0 \mathrm{E}+02 & 2.1+02\end{array}$

Production/Consumption factors:

Produce: $<1 \quad$ Milk: $<1 \quad$ Meat: $<1$

FRACTION OF POPULATION RECEIVING AN INDICATED AVERAGE TOTAL-BODY DOSE COMMITMENT FROM AIRBORNE PATHWAYS

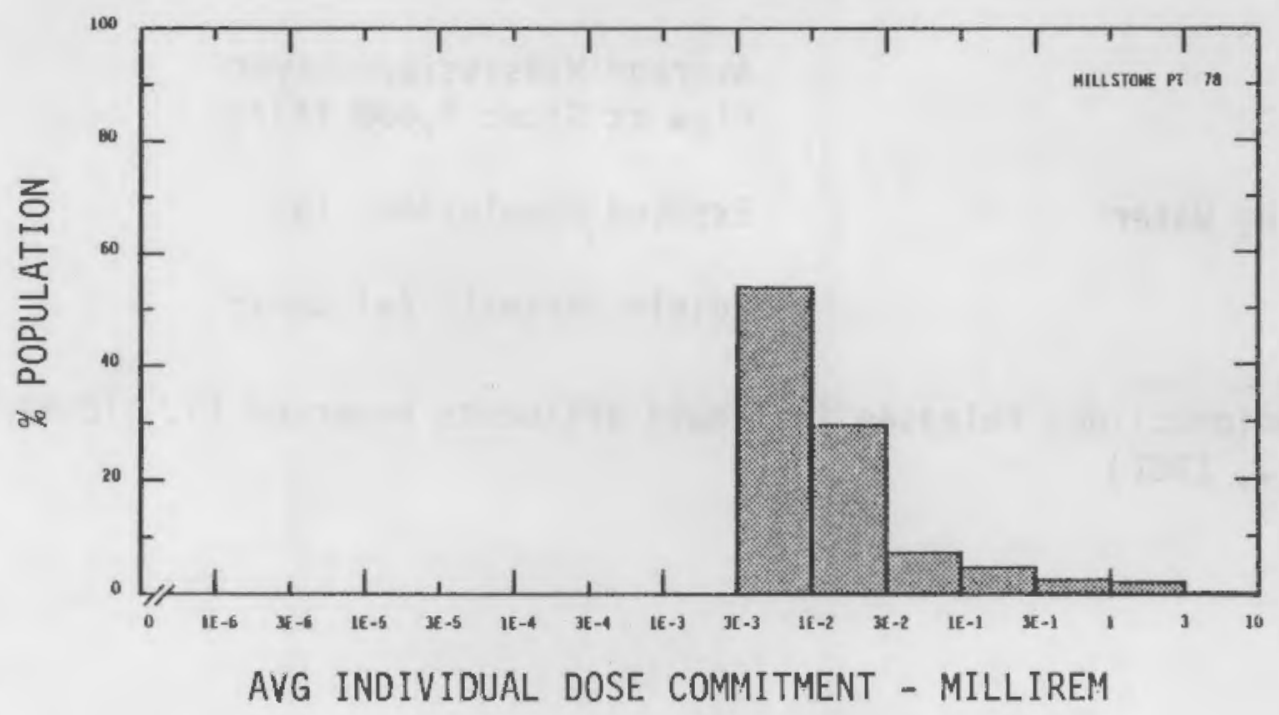


Location: N $\quad$ N $45.33390 \quad$ W 93.84860

POPULATION DATA

Total Population Within 20-to-80-km Region: 2.1E6

Major Metropolitan Centers Within Region:

Center

Minneapolis-St. Paul SMSA St. Cloud

Anoka
Population

$2,100,000$

42,000

14,000
Location

$64 \mathrm{~km} \quad \mathrm{SE}$

$37 \mathrm{~km} \quad \mathrm{NW}$

$35 \mathrm{~km} \quad$ ESE

SITE SPECIFIC DATA - AIRBORNE PATHWAYS

Average Annual State Production

of Crops and Animal Products

In 80-km Radius Circle

Regional Productivity Factor:

Animal Grazing Factor:
Veg: $1.2 \mathrm{E} 8 \mathrm{kilogram}$

Milk: 4.0E8 liter

Meat: $1.1 \mathrm{E} 8 \mathrm{kilogram}$

1

0.5

Meteorology Period of Record: 1 JAN 74 - 31 DEC 74 Recovery: 92\%

SITE SPECIFIC DATA - WATERBORNE PATHWAYS

Average Mississippi River

Flow at Site: $4,600 \mathrm{ft}^{3} / \mathrm{s}$

Drinking Water:

Exposed Population: (a)

Fish:

Edible Harvest: (a) $\mathrm{kg} / \mathrm{yr}$

(a) No radionuclides released in liquid effluents reported (J. Tichler, et al., 1981). 
AVERAGE INDIVIDUAL DOSE-COMMITMENT HISTOGRAM FOR

\section{MONT ICELLO}

Dose Commitments (person-rem) from Liquid Pathways

Total Body GI-LLI Thyroid Bone Liver

$\begin{array}{llllll}\text { Infant } & 0.0 E+00 & 0.0 E+00 & 0.0 E+00 & 0.0 E+00 & 0.0 E+00 \\ \text { Child } & 0.0 E+00 & 0.0 E+00 & 0.0 E+00 & 0.0 E+00 & 0.0 E+00 \\ \text { Teen } & 0.0 E+00 & 0.0 E+00 & 0.0 E+00 & 0.0 E+00 & 0.0 E+00 \\ \text { Adult } & 0.0 E+00 & 0.0 E+00 & 0.0 E+00 & 0.0 E+00 & 0.0 E+00 \\ \text { TOTAL } & 0.0 E+00 & 0.0 E+00 & 0.0 E+00 & 0.0 E+00 & 0.0 E+00\end{array}$

Dose Commitments (person-rem) from Airborne Pathways

\begin{tabular}{|c|c|c|c|c|c|c|}
\hline & Total Body & GI-LLI & Thyroid & Bone & Liver & Lung \\
\hline Infant & $2.7 \mathrm{E}-03$ & $2.6 \mathrm{E}-03$ & $6.0 E-02$ & $1.5 E-03$ & 2. $9 \mathrm{E}-03$ & $2.7 \mathrm{E}-03$ \\
\hline Child & $3.7 \mathrm{E}-02$ & $3.6 \mathrm{E}-02$ & 4.2E-01 & 2. $1 \mathrm{E}-02$ & $3.8 E-02$ & 3. $7 \mathrm{E}-02$ \\
\hline Teen & $2.4 \mathrm{E}-02$ & $2.4 \mathrm{E}-02$ & 1.7E-01 & 1. $3 \mathrm{E}-02$ & $2.4 E-02$ & $2.5 E-02$ \\
\hline Adult & 1. $3 \mathrm{E}-01$ & 1. $3 \mathrm{E}-01$ & $6.4 E-01$ & $6.9 E-02$ & 1. $3 \mathrm{E}-01$ & 1. $4 \mathrm{E}-01$ \\
\hline TOTAL & $2.0 \mathrm{E}-01$ & $2.0 \mathrm{E}-01$ & $1.3 E+00$ & $1.0 E-01$ & 2. $0 E-01$ & 2.0E-01 \\
\hline
\end{tabular}

Production/Consumption factors:

Produce: <1 Milk: $1.4 \quad$ Meat: <1

FRACTION OF POPULATION RECEIVING AN INDICATED AVERAGE

TOTAL-BODY DOSE COMMITMENT FROM AIRBORNE PATHWAYS

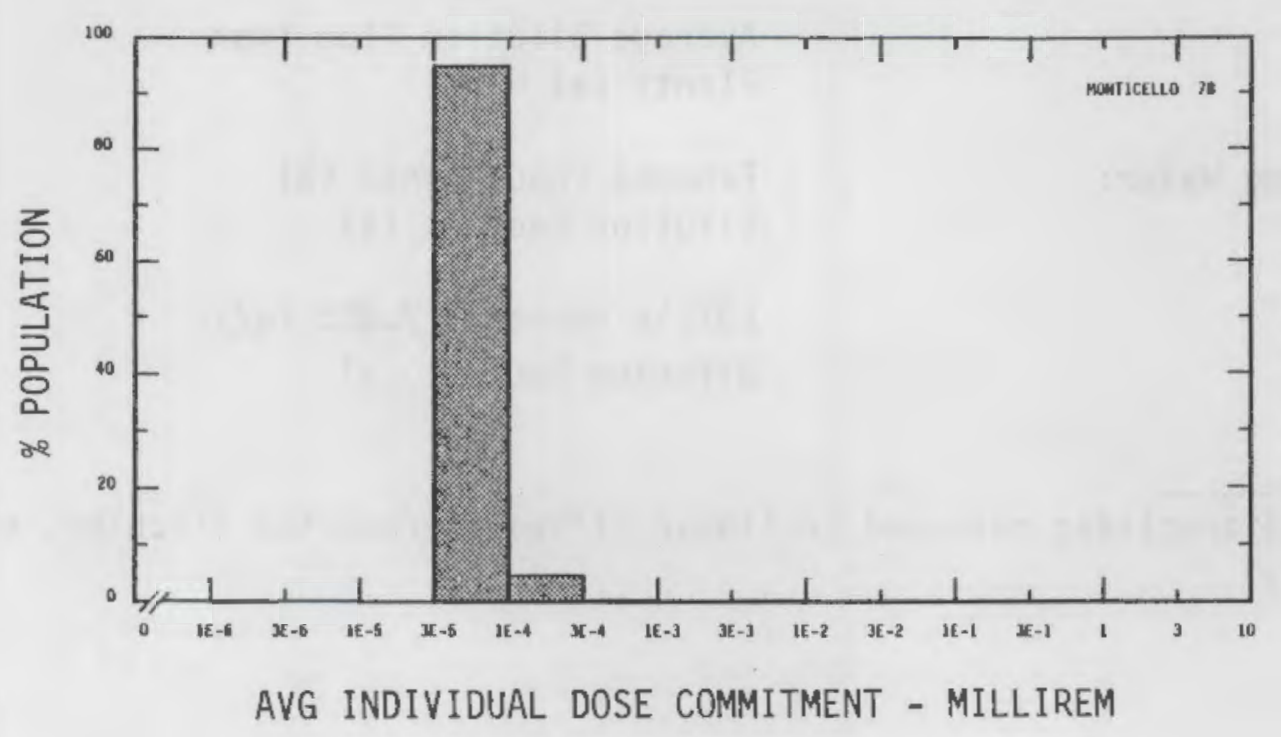


Location: N 43.52170 W 76.39800

POPULATION DATA

Total Population Within 20-to-80-km Region: 8.2E5

Major Metropolitan Centers Within Region:

\begin{tabular}{|c|c|c|}
\hline Center & Population & Locat \\
\hline yracuse SMSA & 620,000 & $54 \mathrm{~km}$ \\
\hline luburn & 34,000 & $66 \mathrm{~km}$ \\
\hline
\end{tabular}

SITE SPECIFIC DATA - AIRBORNE PATHWAYS

Average Annual State Production

of Crops and Animal Products

In 80-km Radius Circle

Regional Productivity Factor:

Animal Grazing Factor:
Veg: $7.6 \mathrm{E} 7 \mathrm{kilogram}$

Milk: 7.0E8 liter

Meat: $3.3 E 7$ kilogram

0.7

0.5

Meteorology Period of Record: 1 JAN 74 - 31 DEC 75 Recovery: 97\%
Average Dilution Flow from
Plant: (a)
Drinking Water:
Exposed Population: (a)
Dilution Factor: (a)
Fish:

(a) No radionuclides released in liquid effluents reported (Tichler, et a1., 1981). 
POPULATION DOSE-COMMITMENT ESTIMATES AND AVERAGE INDIVIDUAL DOSE-COMMITMENT HISTOGRAM FOR

\section{NINE MILE POINT}

Dose Commi tments (person-rem) from Liquid Pathways

Total Body GI-LLI Thyroid Bone Liver

Infant

$0.0 \mathrm{E}+00$

$0.0 \mathrm{E}+00$

$0.0 \mathrm{E}+00$

$0.0 \mathrm{E}+00$

$0.0 E+00$

Child

$0.0 \mathrm{E}+00$

$0.0 \mathrm{E}+00$

$0.0 \mathrm{E}+00$

$0.0 \mathrm{E}+00$

$0.0 \mathrm{E}+00$

Teen

$0.0 \mathrm{E}+00$

$0.0 \mathrm{E}+00$

$0.0 \mathrm{E}+00$

$0.0 \mathrm{E}+00$

$0.0 \mathrm{E}+00$

Adult

$0.0 E+00$

$0.0 \mathrm{E}+00$

$0.0 \mathrm{E}+00$

$0.0 \mathrm{E}+00$

$0.0 \mathrm{E}+00$

TOTAL

$0 . \mathrm{DE}+00$

$0.0 E+00$

$0.0 \mathrm{E}+00$

$0.0 \mathrm{E}+00$

$0.0 \mathrm{E}+00$

Dose Commi tments (person-rem) from Airborne Pathways

Total Body GI-LLI Thyroid Bone Liver Lung

Infant

$1.1 \mathrm{E}-03$

9. $9 \mathrm{E}-04$

$6.2 \mathrm{E}-02$

$1.1 \mathrm{E}-03$

$1.2 \mathrm{E}-03$

1.0E -03

Child

$1.3 \mathrm{E}-02$

$1.2 \mathrm{E}-02$

$4.2 \mathrm{E}-01$

$1.6 \mathrm{E}-02$

$1.4 \mathrm{E}-02$

$1.2 \mathrm{E}-02$

Teen

8.6E-03

8. $5 \mathrm{E}-03$

1.6E-01

8.7E-03

$8.8 \mathrm{E}-03$

8. $8 \mathrm{E}-03$

Adult

4. $9 \mathrm{E}-02$

4. $9 \mathrm{E}-02$

5. $5 \mathrm{E}-01$

4.6E -02

4. $9 \mathrm{E}-02$

$4.9 \mathrm{E}-02$

TOTAL

7.1E-02

7.1E-02

1. $2 E+00$

7.2E-02

7. $3 \mathrm{E}-02$

7.2E-02

Production/Consumption factors:

Produce: $<1 \quad$ Milk: $4.6 \quad$ Meat: $<1$

FRACTION OF POPULATION RECEIVING AN INDICATED AVERAGE TOTAL-BODY DOSE COMMITMENT FROM AIRBORNE PATHWAYS

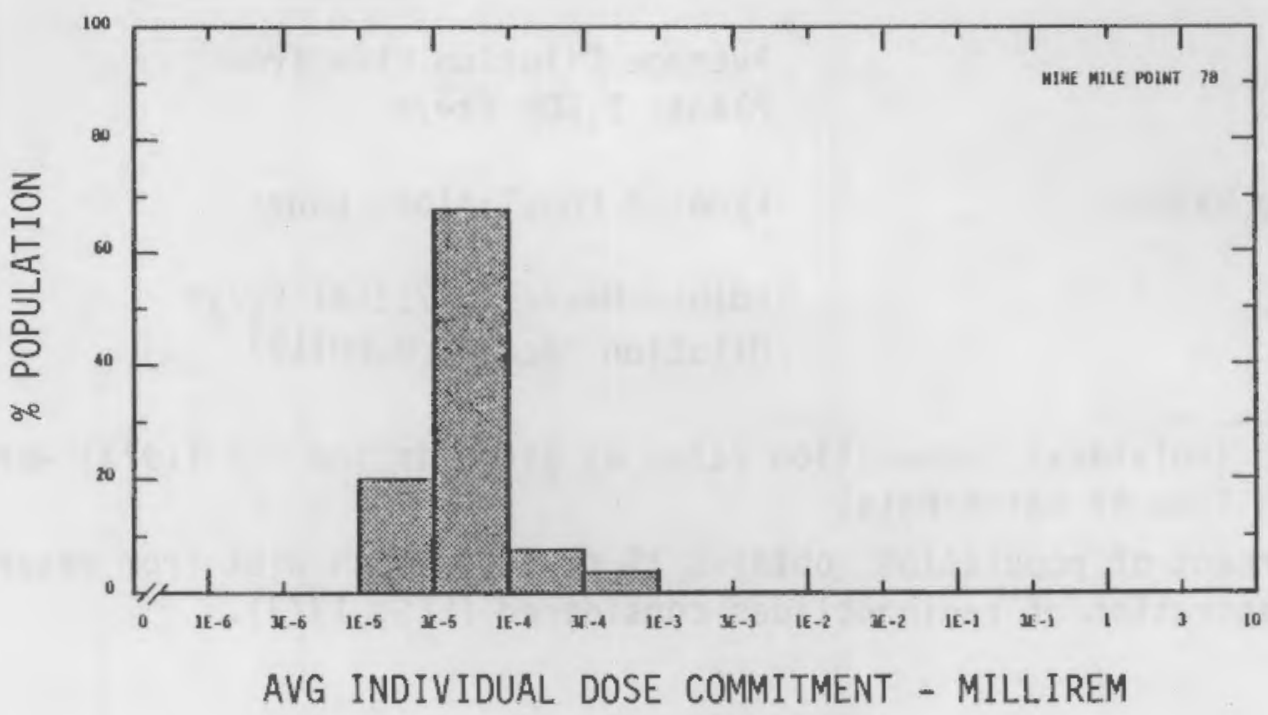




Location: N 38.06310 W 77.78670

POPULATION DATA

Total Population Within 20-to-80-km Region: 9.5E5

Major Metropolitan Centers Within Region:

Center Population Location

$\begin{array}{lrrl}\text { Charlottesville } & 43,000 & 64 \mathrm{~km} & \text { W } \\ \text { Richmond } & 276,000 & 48 \mathrm{~km} & \text { SE } \\ \text { Fredricksburg } & 15,000 & 40 \mathrm{~km} & \text { NE }\end{array}$

SITE SPECIFIC DATA - AIRBORNE PATHWAYS

Average Annual State Production

of Crops and Animal Products

In 80-km Radius Circle

Regional Productivity Factor:

Animal Grazing Factor:
Veg: $3.5 E 7$ kilogram

Milk: $1.5 \mathrm{E} 8$ liter

Meat: 7.4E7 kilogram

0.9

0.7

Meteorology Period of Record: 1 APR 74 - 31 APR 75 Recovery: 99\%

SITE SPECIFIC DATA - WATERBORNE PATHWAYS

Average Dilution Flow from Plant: $1,500 \mathrm{ft}^{3} / \mathrm{s}$

Drinking Water:

Exposed Population: None

Fish:

Edible Harvest: $7.3(\mathrm{a}) \mathrm{kg} / \mathrm{yr}$ Dilution Factor: 0.001 (b)

(a) Average individual consumption rates as given in the FES (1973) were used in lieu of catch data.

(b) Ten percent of population obtains $1 \%$ of their fish diet from reservoir; reconcentration of radionuclides considered (FES, 1973). 
POPULATION DOSE-COMMITMENT ESTIMATES AND AVERAGE INDIVIDUAL DOSE-COMMITMENT HISTOGRAM FOR NORTH ANNA

Dose Commitments (person-rem) from Liquid Pathways Total Body GI-LLI Thyroid Bone Liver

$\begin{array}{llllll}\text { Infant } & 0.0 \mathrm{E}+00 & 0.0 \mathrm{E}+00 & 0.0 \mathrm{E}+00 & 0.0 \mathrm{E}+00 & 0.0 \mathrm{E}+00 \\ \text { Child } & 1.2 \mathrm{E}-02 & 2.1 \mathrm{E}-03 & 2.8 \mathrm{E}-03 & 6.2 \mathrm{E}-02 & 6.8 \mathrm{E}-02 \\ \text { Teen } & 2.2 \mathrm{E}-02 & 4.1 \mathrm{E}-03 & 2.1 \mathrm{E}-03 & 3.7 \mathrm{E}-02 & 5.7 \mathrm{E}-02 \\ \text { Adult } & 2.4 \mathrm{E}-01 & 3.6 \mathrm{E}-02 & 1.4 \mathrm{E}-02 & 2.1 \mathrm{E}-01 & 3.4 \mathrm{E}-01 \\ \text { TOTAL } & 2.7 \mathrm{E}-01 & 4.2 \mathrm{E}-02 & 1.9 \mathrm{E}-02 & 3.1 \mathrm{E}-01 & 4.7 \mathrm{E}-01\end{array}$

Dose Commitments (person-rem) from Airborne Pathways Total Body Gl-LLI Thyroid Bone Liver Lung

$\begin{array}{lllllll}\text { Infant } & 3.1 \mathrm{E}-03 & 3.1 \mathrm{E}-03 & 2.4 \mathrm{E}-02 & 3.1 \mathrm{E}-03 & 3.2 \mathrm{E}-03 & 3.2 \mathrm{E}-03 \\ \text { Child } & 3.4 \mathrm{E}-02 & 3.4 \mathrm{E}-02 & 1.5 \mathrm{E}-01 & 3.4 \mathrm{E}-02 & 3.5 \mathrm{E}-02 & 3.6 \mathrm{E}-02 \\ \text { Teen } & 2.5 \mathrm{E}-02 & 2.5 \mathrm{E}-02 & 6.8 \mathrm{E}-02 & 2.5 \mathrm{E}-02 & 2.5 \mathrm{E}-02 & 2.8 \mathrm{E}-02 \\ \text { Adult } & 1.5 \mathrm{E}-01 & 1.5 \mathrm{E}-01 & 2.9 \mathrm{E}-01 & 1.5 \mathrm{E}-01 & 1.5 \mathrm{E}-01 & 1.6 \mathrm{E}-01 \\ \text { TOTAL } & 2.1 \mathrm{E}-01 & 2.1 \mathrm{E}-01 & 5.3 \mathrm{E}-01 & 2.1 \mathrm{E}-01 & 2.1 \mathrm{E}-01 & 2.3 \mathrm{E}-01\end{array}$

Production/Consumption factors:
Produce: $<1$
Milk: 1.1
Meat: $<1$

FRACTION OF POPULATION RECEIVING AN INDICATED AVERAGE TOTAL-BODY DOSE COMMITMENT FROM AIRBORNE PATHWAYS

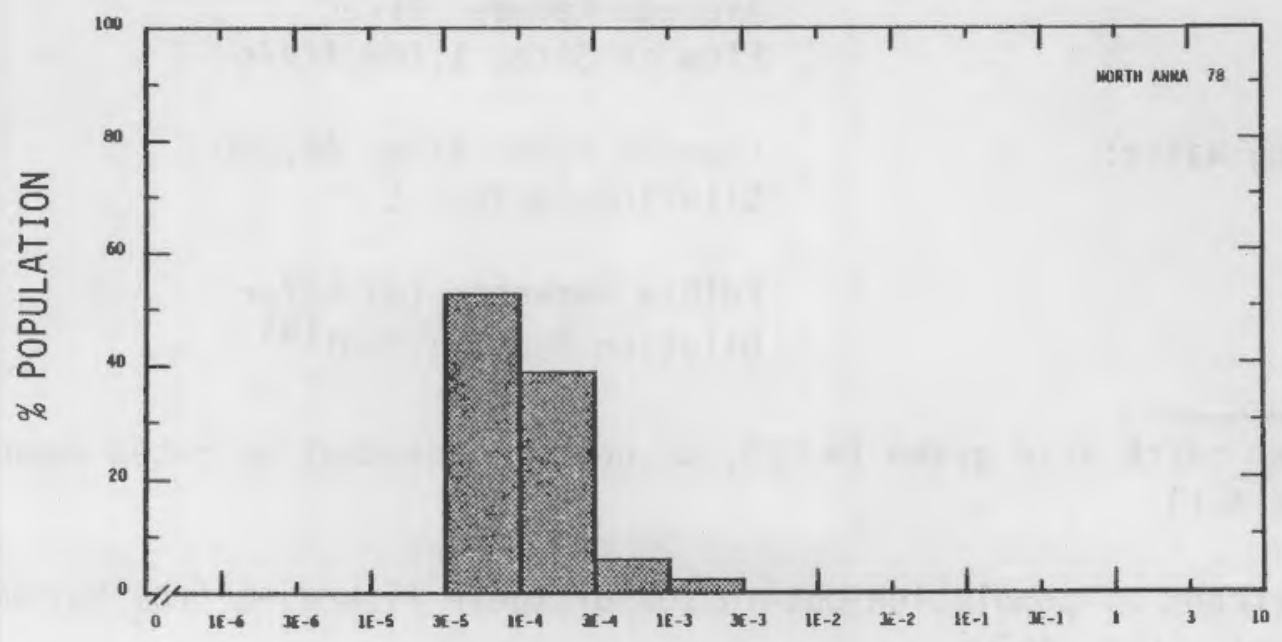

AVG INDIVIDUAL DOSE COMMITMENT - MILLIREM 

Location:
N $\quad 34.75000$
W 83.04580

POPULATION DATA

Total Population Within 20-to-80-km Region: 7.5E5

\begin{tabular}{|c|c|c|}
\hline Center & Population & Location \\
\hline Anderson & 31,000 & $39 \mathrm{~km}$ \\
\hline Greenville & 69,000 & $47 \mathrm{~km}$ \\
\hline
\end{tabular}

SITE SPECIFIC DATA - AIRBORNE PATHWAYS

Average Annual State Production

of Crops and Animal Products

In 80-km Radius Circle

Regional Productivity Factor:

Animal Grazing Factor:

Meteorology Period of Record: 1 JAN 75 - 31 DEC 75 Recovery: $86 \%$
Veg: 7.4E6 kilogram

Milk: 5.7E7 liter

Meat: 5.0E7 kilogram

1

0.7

SITE SPECIFIC DATA - WATERBORNE PATHWAYS

Average Keowee River

Flow at Site: $1,100 \mathrm{ft}^{3} / \mathrm{s}$

Drinking Water:

Exposed Population: 44,000

Dilution Factor: 1

Fish:

Edible Harvest: (a) $\mathrm{kg} / \mathrm{yr}$

Dilution Factor: $0.01(b)$

(a) No fish catch data given in FES, so generic consumption rates used (Table A-1).

(b) Ten percent of population obtain $10 \%$ of their fish diet from Hartwell Reservoir (FES, 1972). 
POPULATION DOSE-COMMITMENT ESTIMATES AND AVERAGE INDIVIDUAL DOSE-COMMITMENT HISTOGRAM FOR

OCONEE 1,2 AND 3

Dose Commitments (person-rem) from Liquid Pathways

$\underline{\text { Total Body GI-LLI } \underline{\text { Thyroid }} \text { Bone }}$

$\begin{array}{llllll}\text { Infant } & 5.5 \mathrm{E}-02 & 4.6 \mathrm{E}-02 & 1.4 \mathrm{E}+00 & 9.2 \mathrm{E}-02 & 1.2 \mathrm{E}-01 \\ \text { Child } & 9.9 \mathrm{E}-01 & 6.3 \mathrm{E}-01 & 1.0 \mathrm{E}+01 & 2.6 \mathrm{E}+00 & 2.9 \mathrm{E}+00 \\ \text { Teen } & 9.1 \mathrm{E}-01 & 4.0 \mathrm{E}-01 & 3.2 \mathrm{E}+00 & 1.2 \mathrm{E}+00 & 1.9 \mathrm{E}+00 \\ \text { Adult } & 9.4 \mathrm{E}+00 & 3.5 \mathrm{E}+00 & 2.3 \mathrm{E}+01 & 7.3 \mathrm{E}+00 & 1.2 \mathrm{E}+01 \\ \text { TOTAL } & 1.1 \mathrm{E}+01 & 4.6 \mathrm{E}+00 & 3.7 \mathrm{E}+01 & 1.1 \mathrm{E}+01 & 1.7 \mathrm{E}+01\end{array}$

Dose Commitments (person-rem) from Airborne Pathways

Total Body GI-LLI Thyroid Bone Liver Lung

$\begin{array}{lllllll}\text { Infant } & 1.1 \mathrm{E}-02 & 1.1 \mathrm{E}-02 & 5.5 \mathrm{E}-02 & 1.0 \mathrm{E}-02 & 1.1 \mathrm{E}-02 & 1.1 \mathrm{E}-02 \\ \text { Child } & 1.2 \mathrm{E}-01 & 1.2 \mathrm{E}-01 & 3.7 \mathrm{E}-01 & 1.2 \mathrm{E}-01 & 1.2 \mathrm{E}-01 & 1.3 \mathrm{E}-01 \\ \text { Teen } & 8.6 \mathrm{E}-02 & 8.6 \mathrm{E}-02 & 2.0 \mathrm{E}-01 & 8.4 \mathrm{E}-02 & 8.6 \mathrm{E}-02 & 1.0 \mathrm{E}-01 \\ \text { Adult } & 5.2 \mathrm{E}-01 & 5.2 \mathrm{E}-01 & 9.2 \mathrm{E}-01 & 5.1 \mathrm{E}-01 & 5.2 \mathrm{E}-01 & 5.7 \mathrm{E}-01 \\ \text { TOTAL } & 7.3 \mathrm{E}-01 & 7.4 \mathrm{E}-01 & 1.5 \mathrm{E}+00 & 7.2 \mathrm{E}-01 & 7.4 \mathrm{E}-01 & 8.2 \mathrm{E}-01\end{array}$

Production/Consumption factors:

Produce: $<1 \quad$ Milk: <1 Meat: <1

FRACTION OF POPULATION RECEIVING AN INDICATED AVERAGE TOTAL-BODY DOSE COMMITMENT FROM AIRBORNE PATHWAYS

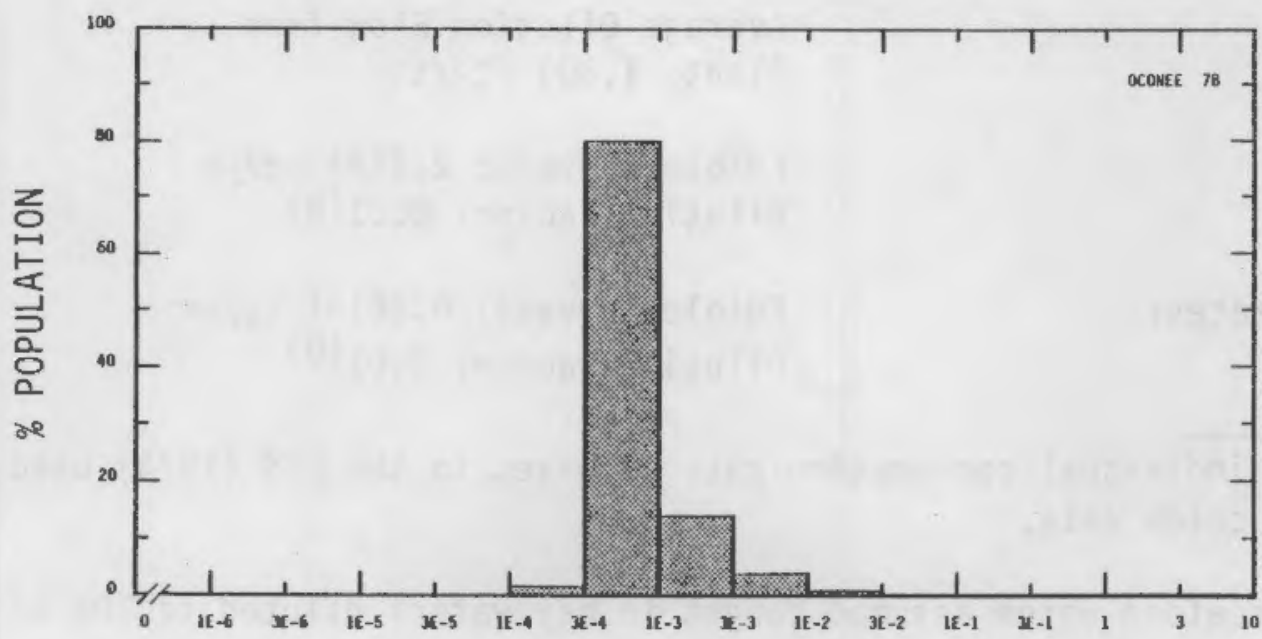

AVG INDIVIDUAL. DOSE COMMITMENT - MILLIREM 
Site: OYSTER CREEK

OYSTER CREEK, NEH JERSEY

Location: N 39.81390 W 74.21140

POPULATION DATA

Total Population Within 20-to-80-km Region: 3.3E6

Major Metropolitan Centers Within Region:

Center Population Location

Atlantic City $\quad 49,000 \quad 56 \mathrm{~km} \quad$ SSH

Trenton $110,000 \quad 64 \mathrm{~km}$ NW

SITE SPECIFIC DATA - AIRBORNE PATHWAYS

Average Annual State Production

of Crops and Animal Products

In 80-km Radius Circle

Regional Productivity Factor:

Animal Grazing Factor:

Meteorology Period of Record: 15 FEB 66 - 31 DEC 68 Recovery: $63 \%$

SITE SPECIFIC DATA - WATERBORNE PATHWAYS

Average Dilution Flow from

Plant: $1,800 \mathrm{ft}^{3} / \mathrm{s}$

Fish:

Edible Harvest: 2.1 (a) $\mathrm{kg} / \mathrm{yr}$

Dilution Factor: 0.01 (b)

Invertebrates:

Edible Harvest: $0.96(\mathrm{a}) \mathrm{kg} / \mathrm{yr}$

Dilution Factor: 0.01 (b)

(a) Average individual consumption rate as given in the FES (1974) used in lieu of catch data.

(b) $10 \%$ of seafood eaten assumed caught in bay waters diluted to $10 \%$ of that of discharge canal (FES, 1974). 
POPULATION DOSE-COMMITMENT ESTIMATES AND AVERAGE INDIVIDUAL DOSE-COMMITMENT HISTOGRAM FOR OYSTER CREEK

Dose Commitments (person-rem) from Liquid Pathways Total Body GI-LLI Thyroid Bone Liver

$\begin{array}{llllll}\text { Infant } & 0.0 \mathrm{E}+00 & 0.0 \mathrm{E}+00 & 0.0 \mathrm{E}+00 & 0.0 \mathrm{E}+00 & 0.0 \mathrm{E}+00 \\ \text { Child } & 1.8 \mathrm{E}-03 & 6.0 \mathrm{E}-03 & 1.7 \mathrm{E}-04 & 1.2 \mathrm{E}-03 & 2.4 \mathrm{E}-03 \\ \text { Teen } & 1.4 \mathrm{E}-03 & 1.2 \mathrm{E}-02 & 1.3 \mathrm{E}-04 & 7.7 \mathrm{E}-04 & 2.1 \mathrm{E}-03 \\ \text { Adult } & 9.2 \mathrm{E}-03 & 1.1 \mathrm{E}-01 & 8.4 \mathrm{E}-04 & 4.7 \mathrm{E}-03 & 1.3 \mathrm{E}-02 \\ \text { TOTAL } & 1.2 \mathrm{E}-02 & 1.2 \mathrm{E}-01 & 1.1 \mathrm{E}-03 & 6.7 \mathrm{E}-03 & 1.8 \mathrm{E}-02\end{array}$

Dose Commitments (person-rem) from Airborne Pathways Total Body GI-LLI Thyroid Bone Liver Lung

$\begin{array}{lllllll}\text { Infant } & 1.6 \mathrm{E}+00 & 1.6 \mathrm{E}+00 & 6.7 \mathrm{E}+00 & 1.6 \mathrm{E}+00 & 1.6 \mathrm{E}+00 & 1.7 \mathrm{E}+00 \\ \text { Child } & 1.7 \mathrm{E}+01 & 1.8 \mathrm{E}+01 & 5.3 \mathrm{E}+01 & 1.8 \mathrm{E}+01 & 1.7 \mathrm{E}+01 & 1.9 \mathrm{E}+01 \\ \text { Teen } & 1.3 \mathrm{E}+01 & 1.3 \mathrm{E}+01 & 2.9 \mathrm{E}+01 & 1.3 \mathrm{E}+01 & 1.3 \mathrm{E}+01 & 1.4 \mathrm{E}+01 \\ \text { Adult } & 7.7 \mathrm{E}+01 & 7.8 \mathrm{E}+01 & 1.4 \mathrm{E}+02 & 7.8 \mathrm{E}+01 & 7.7 \mathrm{E}+01 & 8.2 \mathrm{E}+01 \\ \text { TOTAL } & 1.1 \mathrm{E}+02 & 1.1 \mathrm{E}+02 & 2.3 \mathrm{E}+02 & 1.1 \mathrm{E}+02 & 1.1 \mathrm{E}+02 & 1.2 \mathrm{E}+02\end{array}$

Production/Consumption factors:

Produce: $<1 \quad$ Milk: $<1 \quad$ Meat: $<1$

FRACTION OF POPULATION RECEIVING AN INOICATED AVERAGE TOTAL-BODY DOSE COMMITMENT FROM AIRBORNE PATHWAYS

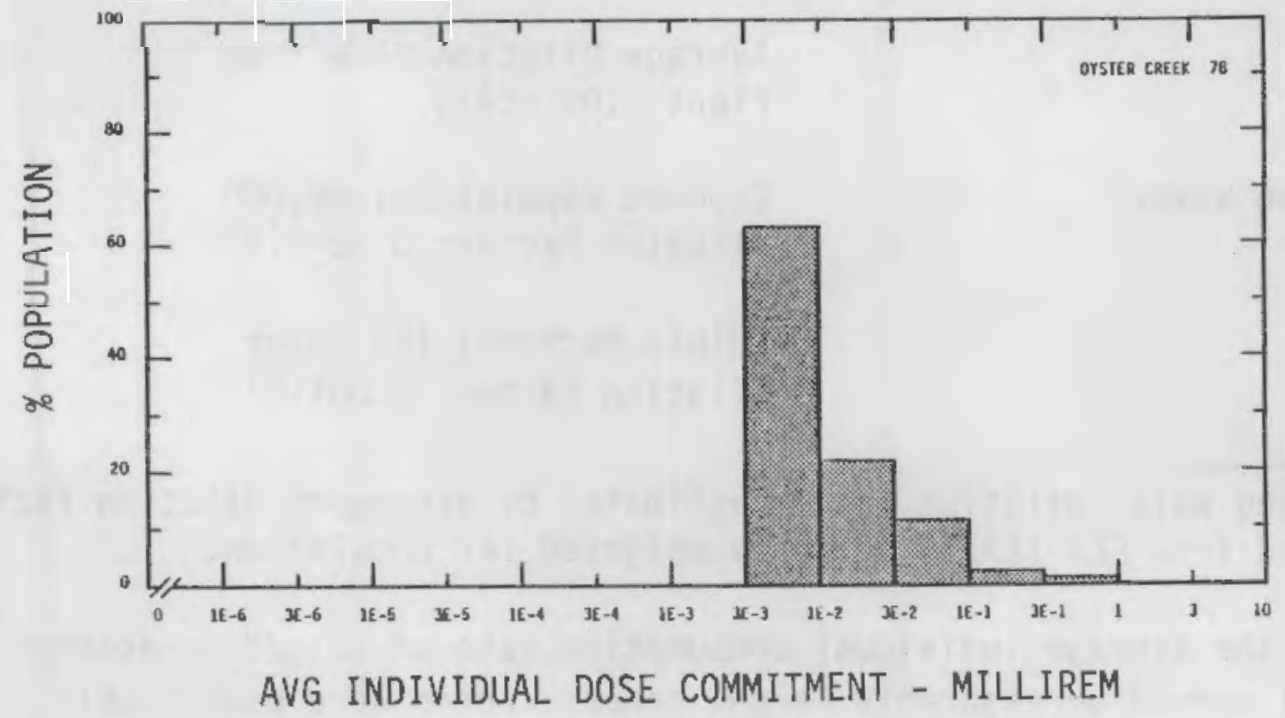


Location: $\quad \mathrm{N} 42.3233^{\circ}$ W $86.3142^{\circ}$

POPULATION DATA

Total Population Within 20-to-80-km Region: 1.0E6

Major Metropolitan Centers Within Region:

Center

Kal amazoo

South Bend SMSA

Michigan City
Population

88,000

290,000

41,000
Location

$56 \mathrm{~km} \quad \mathrm{E}$

$72 \mathrm{~km} \quad \mathrm{~S}$

$66 \mathrm{~km} \quad \mathrm{SW}$

SITE SPECIFIC DATA - AIRBORNE PATHWAYS

Average Annual State Production

of Crops and Animal Products

In 80-km Radius Circle

Regional Productivity Factor:

Animal Grazing Factor:
Veg: $6.8 \mathrm{E} 7 \mathrm{kilogram}$

Milk: 2.9E8 liter

Meat: $4.5 E 7$ kilogram

0.6

0.5

Meteorology Period of Record: 1 SEP $73-31$ AUG 74 Recovery: 67\%

SITE SPECIFIC DATA - WATERBORNE PATHWAYS

Average Dilution Flow from

Plant: $109 \mathrm{ft}^{3} / \mathrm{s}$

Drinking Water:

Exposed Population: 48,000 Dilution Factor: $3.5 \mathrm{E}-3(\mathrm{a})$

Fish:

Edible Harvest: (b) $\mathrm{kg} / \mathrm{yr}$

Dilution Factor: $0.001(\mathrm{~b})$

(a) Drinking water dilution factor estimated by averaging dilution factor derived from FES (1972) suitably weighted for population.

(b) Since the average individual consumption rate of $20 \mathrm{~g} / \mathrm{d}$ as assumed in FES (1972) seemed unreasonably large, generic rates were used (Table A-1). 
POPULATION DOSE-COMMITMENT ESTIMATES AND

AVERAGE INOIVIDUAL DOSE-COMMITMENT HISTOGRAM FOR

PALISADES

Dose Commitments (person-rem) from Liquid Pathways

Total Body GI-LLI Thyroid Bone Liver

$\begin{array}{llllll}\text { Infant } & 1.4 \mathrm{E}-04 & 1.4 \mathrm{E}-04 & 1.5 \mathrm{E}-04 & 2.2 \mathrm{E}-05 & 1.6 \mathrm{E}-04 \\ \text { Child } & 5.6 \mathrm{E}-03 & 5.0 \mathrm{E}-03 & 1.8 \mathrm{E}-03 & 2.4 \mathrm{E}-02 & 2.7 \mathrm{E}-02 \\ \text { Teen } & 8.3 \mathrm{E}-03 & 7.8 \mathrm{E}-03 & 7.2 \mathrm{E}-04 & 1.4 \mathrm{E}-02 & 2.1 \mathrm{E}-02 \\ \text { Adult } & 8.9 \mathrm{E}-02 & 6.8 \mathrm{E}-02 & 5.9 \mathrm{E}-03 & 8.2 \mathrm{E}-02 & 1.3 \mathrm{E}-01 \\ \text { TOTAL } & 1.0 \mathrm{E}-01 & 8.2 \mathrm{E}-02 & 8.6 \mathrm{E}-03 & 1.2 \mathrm{E}-01 & 1.8 \mathrm{E}-01\end{array}$

Dose Commitments (person-rem) from Airborne Pathways

Total Body GI-LLI Thyroid Bone Liver Lung

$\begin{array}{lllllll}\text { Infant } & 8.0 \mathrm{E}-05 & 7.2 \mathrm{E}-05 & 6.1 \mathrm{E}-03 & 8.4 \mathrm{E}-05 & 1.0 \mathrm{E}-04 & 8.6 \mathrm{E}-05 \\ \text { Child } & 9.2 \mathrm{E}-04 & 8.7 \mathrm{E}-04 & 3.8 \mathrm{E}-02 & 8.5 \mathrm{E}-04 & 1.1 \mathrm{E}-03 & 1.1 \mathrm{E}-03 \\ \text { Teen } & 6.3 \mathrm{E}-04 & 6.3 \mathrm{E}-04 & 1.5 \mathrm{E}-02 & 5.3 \mathrm{E}-04 & 6.8 \mathrm{E}-04 & 8.1 \mathrm{E}-04 \\ \text { Adult } & 3.6 \mathrm{E}-03 & 3.7 \mathrm{E}-03 & 5.0 \mathrm{E}-02 & 3.0 \mathrm{E}-03 & 3.7 \mathrm{E}-03 & 4.3 \mathrm{E}-03 \\ \text { TOTAL } & 5.2 \mathrm{E}-03 & 5.3 \mathrm{E}-03 & 1.1 \mathrm{E}-01 & 4.4 \mathrm{E}-03 & 5.5 \mathrm{E}-03 & 6.2 \mathrm{E}-03\end{array}$

Production/Consumption factors:

Produce: <1 Milk: 1.3 Meat: <1

FRACTION OF POPULATION RECEIVING AN INDICATED AVERAGE TOTAL-BODY DOSE COMMITMENT FROM AIRBORNE PATHWAYS

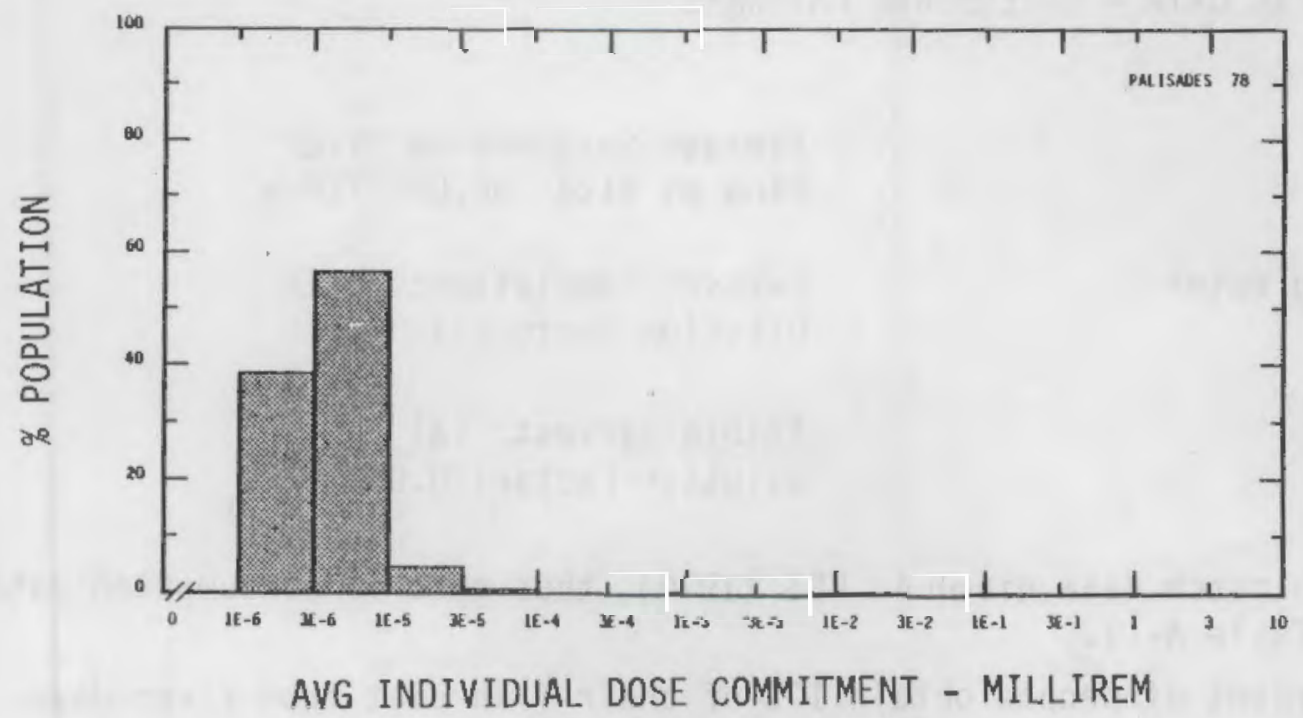


Site: PEACH BOTTOM

YORK COUNTY, PENNSYLVANIA

Location: N $39.7592^{\circ}$ W 76.26890

POPULATION DATA

Total Population Within 20-to-80-km Region: 4.1E6

Major Metropolitan Centers Within Region:

Center

Lancaster SMSA

Baltimore SMSA

Wilmington SMSA

Reading SMSA
Population

320,000
$2,100,000$
500,000
290,000

Location

$31 \mathrm{~km} \mathrm{~N}$

$58 \mathrm{~km} \quad \mathrm{SW}$

$64 \mathrm{~km} \quad \mathrm{E}$

$72 \mathrm{~km} \quad$ NNE

SITE SPECIFIC DATA - AIRBORNE PATHWAYS

Average Annual State Production

of Crops and Animal Products

In $80-\mathrm{km}$ Radius Circle

Regional Productivity Factor:

Animal Grazing Factor:
Yeg: $5.3 E 7$ kilogram

Milk: $5.3 \mathrm{E} 8$ liter

Meat: $5.4 \mathrm{E} 7 \mathrm{kilogram}$

0.95

0.6

Meteorology Period of Record: 1 AUG $67-31$ JUL 71 Recovery: $72 \%$

Average Susquehanna River

Flow at site: $36,000 \mathrm{ft}^{3} / \mathrm{s}$

Drinking Water:

Fish:

Exposed Population: 2.1E6

Dilution Factor: 1

Edible Harvest: (a) $\mathrm{kg} / \mathrm{yr}$

Dilution Factor: $0.001(\mathrm{~b})$

(a) No fish catch data given in FES (1974), thus generic consumption rates used (Table A-1).

(b) One percent of people obtain $10 \%$ of their fish diet from river downstream from plant (FES, 1973). 
POPULATION DOSE-COMMITMENT ESTIMATES AND

AVERAGE INDIVIDUAL DOSE-COMMITMENT HISTOGRAM FOR

PEACH BOTTOM 2 AND 3

Dose Commitments (person-rem) from Liquid Pathways

Total Body GI-LLI Thyroid Bone Liver

Infant

Child

4.7E-02

1.1E-02

$5.4 \mathrm{E}-01$

2.6E-01

4.1E-01

Teen

Adult

8.6E-01

1.0E-01

$3.8 \mathrm{E}+00$

$2.8 E+00$

3. $9 E+00$

$6.8 \mathrm{E}-01$

6.8E-02

$1.1 \mathrm{E}+00$

7. $6 \mathrm{E}-01$

1. $5 \mathrm{E}+00$

$7.7 E+00$

6. $0 \mathrm{E}-01$

8. $0 E+00$

4. $8 \mathrm{E}+00$

9. $7 E+00$

TOTAL

9. $3 \mathrm{E}+00$

7. $8 \mathrm{E}-01$

1. $3 E+01$

8. $6 \mathrm{E}+00$

1. $6 \mathrm{E}+01$

Dose Commitments (person-rem) from Airborne Pathways Total Body GI-LLI Thyroid Bone Liver Lung

$\begin{array}{lllllll}\text { Infant } & 7.7 \mathrm{E}-02 & 7.7 \mathrm{E}-02 & 2.5 \mathrm{E}-01 & 7.7 \mathrm{E}-02 & 7.8 \mathrm{E}-02 & 8.2 \mathrm{E}-02 \\ \text { Child } & 8.5 \mathrm{E}-01 & 8.5 \mathrm{E}-01 & 1.9 \mathrm{E}+00 & 8.5 \mathrm{E}-01 & 8.6 \mathrm{E}-01 & 9.4 \mathrm{E}-01 \\ \text { Teen } & 6.2 \mathrm{E}-01 & 6.2 \mathrm{E}-01 & 1.1 \mathrm{E}+00 & 6.2 \mathrm{E}-01 & 6.2 \mathrm{E}-01 & 7.3 \mathrm{E}-01 \\ \text { Adult } & 3.8 \mathrm{E}+00 & 3.8 \mathrm{E}+00 & 5.6 \mathrm{E}+00 & 3.7 \mathrm{E}+00 & 3.8 \mathrm{E}+00 & 4.1 \mathrm{E}+00 \\ \text { TOTAL } & 5.3 \mathrm{E}+00 & 5.3 \mathrm{E}+00 & 8.9 \mathrm{E}+00 & 5.3 \mathrm{E}+00 & 5.3 \mathrm{E}+00 & 5.9 \mathrm{E}+00\end{array}$

Production/Consumption factors:
Produce: $<1$
Milk: $<1$
Meat: $<1$

FRACTION OF POPUL.ATION RECEIVING AN INDICATED AVERAGE TOTAL-BODY DOSE COMMITMENT FROM AIRBORNE PATHWAYS

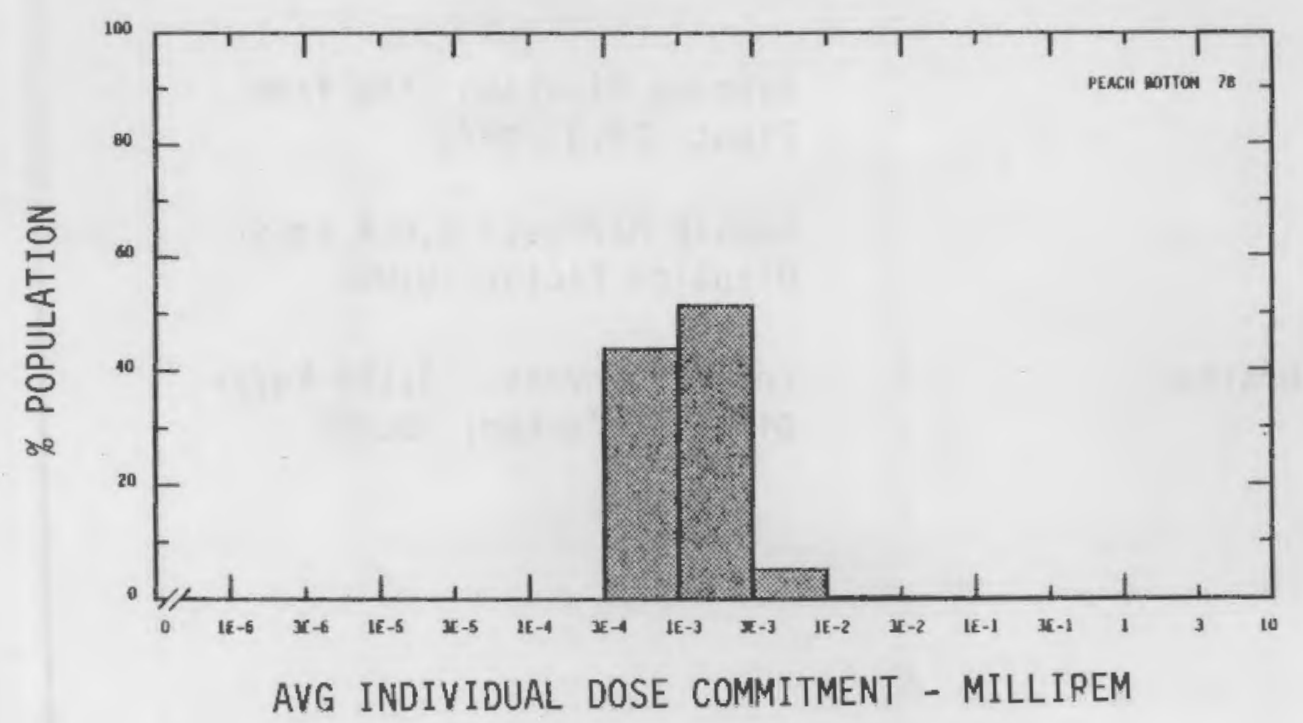




Location: N 41.94440 W $70.5778^{\circ}$

POPULATION DATA

Total Population Within 20-to-80-km Region: 4.3E6

Major Metropolitan Centers Within Region:

Center Population Location

$\begin{array}{lrrl}\text { Boston SMSA } & 3,400,000 & 64 \mathrm{~km} & \text { NW } \\ \text { Providence SMSA } & 780,000 & 70 \mathrm{~km} & \text { WSW } \\ \text { Brockton } & 90,000 & 40 \mathrm{~km} & \text { WNW } \\ \text { New Bedford } & 100,000 & 45 \mathrm{~km} & \text { SW }\end{array}$

SITE SPECIFIC DATA - AIRBORNE PATHWAYS

Average Annual State Production

of Crops and Animal Products

In 80-km Radius Circle

Regional Productivity Factor:

Animal Grazing Factor:

Meteorology Period of Record: 1 MAY 74 - 30 APR 75 Recovery: 93\%
Veg: 2.0E7 kilogram

Milk: $2.6 \mathrm{E} 8$ liter

Meat: $1.6 \mathrm{E} 7 \mathrm{kilogram}$

0.3

0.6

SITE SPECIFIC DATA - WATERBORNE PATHWAYS

Average Dilution Flow from

Plant: $14.8 \mathrm{ft}^{3} / \mathrm{s}$

Fish:

Edible Harvest: $2.6 \mathrm{E} 4 \mathrm{~kg} / \mathrm{yr}$

Dilution Factor: 0.001

Invertebrates:

Edible Harvest: $3.1 E 4 \mathrm{~kg} / \mathrm{yr}$

Dilution Factor: 0.002 
AVERAGE INDIVIDUAL DOSE-COMMITMENT HISTOGRAM FOR

PILGRIM

Dose Commitments (person-rem) from Liquid Pathways

Total Body GI-LLI $\underline{\text { Thyroid Bone }}$

$\begin{array}{llllll}\text { Infant } & 0.0 \mathrm{E}+00 & 0.0 \mathrm{E}+00 & 0.0 \mathrm{E}+00 & 0.0 \mathrm{E}+00 & 0.0 \mathrm{E}+00 \\ \text { Child } & 7.9 \mathrm{E}-03 & 7.8 \mathrm{E}-03 & 6.9 \mathrm{E}-06 & 3.9 \mathrm{E}-03 & 9.9 \mathrm{E}-03 \\ \text { Teen } & 5.5 \mathrm{E}-03 & 1.6 \mathrm{E}-02 & 4.9 \mathrm{E}-06 & 2.7 \mathrm{E}-03 & 8.9 \mathrm{E}-03 \\ \text { Adult } & 3.4 \mathrm{E}-02 & 1.4 \mathrm{E}-01 & 3.2 \mathrm{E}-05 & 1.8 \mathrm{E}-02 & 5.5 \mathrm{E}-02 \\ \text { TOTAL } & 4.7 \mathrm{E}-02 & 1.7 \mathrm{E}-01 & 4.4 \mathrm{E}-05 & 2.4 \mathrm{E}-02 & 7.4 \mathrm{E}-02\end{array}$

Dose Commitments (person-rem) from Airborne Pathways Total Body GI-LLI Thyroid Bone Liver Lung

$\begin{array}{lllllll}\text { Infant } & 1.0 \mathrm{E}-01 & 1.0 \mathrm{E}-01 & 1.6 \mathrm{E}-01 & 1.0 \mathrm{E}-01 & 1.0 \mathrm{E}-01 & 1.1 \mathrm{E}-01 \\ \text { Child } & 1.2 \mathrm{E}+00 & 1.2 \mathrm{E}+00 & 1.6 \mathrm{E}+00 & 1.2 \mathrm{E}+00 & 1.2 \mathrm{E}+00 & 1.2 \mathrm{E}+00 \\ \text { Teen } & 8.5 \mathrm{E}-01 & 8.5 \mathrm{E}-01 & 1.0 \mathrm{E}+00 & 8.5 \mathrm{E}-01 & 8.5 \mathrm{E}-01 & 9.0 \mathrm{E}-01 \\ \text { Adult } & 5.2 \mathrm{E}+00 & 5.2 \mathrm{E}+00 & 5.9 \mathrm{E}+00 & 5.1 \mathrm{E}+00 & 5.2 \mathrm{E}+00 & 5.3 \mathrm{E}+00 \\ \text { TOTAL } & 7.3 \mathrm{E}+00 & 7.3 \mathrm{E}+00 & 8.6 \mathrm{E}+00 & 7.2 \mathrm{E}+00 & 7.3 \mathrm{E}+00 & 7.5 \mathrm{E}+00\end{array}$

Production/Consumption factors:

Produce: <1 Milk: $<1 \quad$ Meat: $<1$

FRACTION OF POPULATION RECEIVING AN INDICATED AVERAGE TOTAL-BODY DOSE COMMITMENT FROM AIRBORNE PATHWAYS

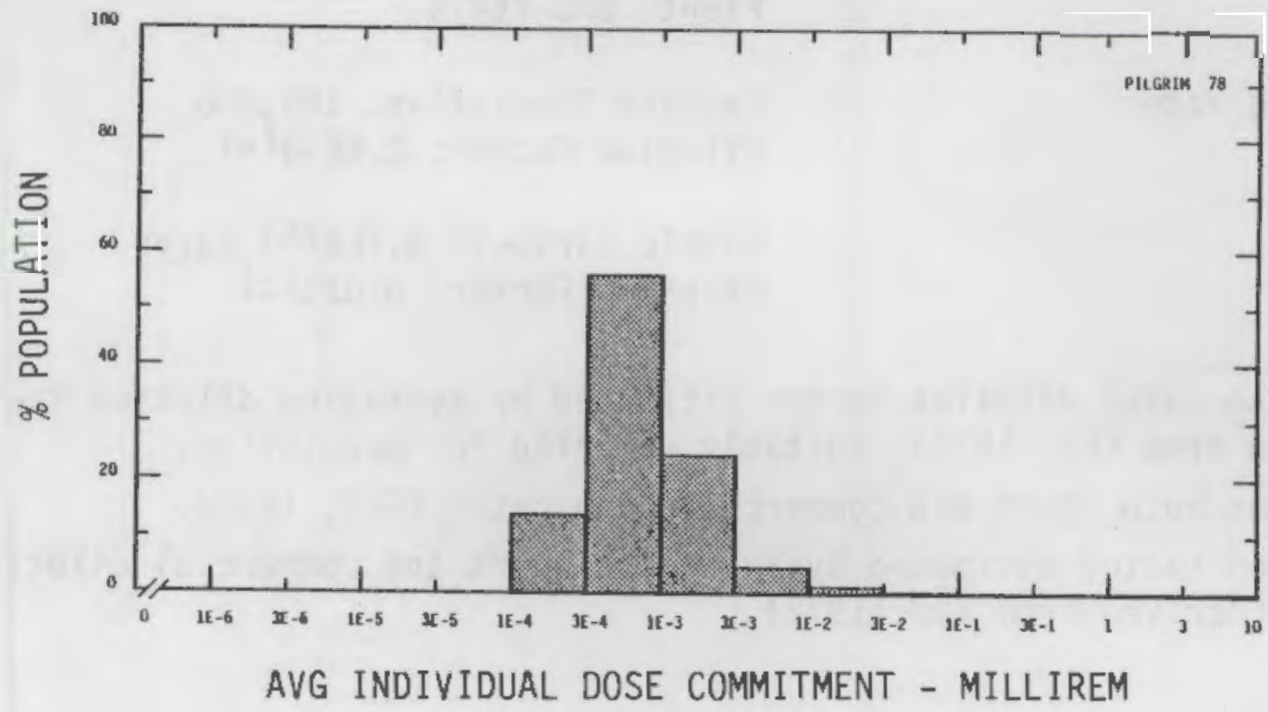


Location: N $44.27640 \quad$ W 87.51860

POPULATION DATA

Total Population Within 20-to-80-km Region: 6.0E5

Major Metropolitan Centers Within Region:
Center
Population
Location

Sheboygan

51,000

Manitowoc

35,000

Greenbay

93,000

Appleton

60,000

$56 \mathrm{~km} \quad \mathrm{SSW}$

$19 \mathrm{~km} \quad \mathrm{SSW}$

$48 \mathrm{~km} \quad \mathrm{NW}$

$72 \mathrm{~km} \mathrm{~W}$

SITE SPECIFIC DATA - AIRBORNE PATHWAYS

Average Annual State Production

of Crops and Animal Products

In 80-km Radius Circle

Regional Productivity Factor:

Animal Grazing Factor:
Veg: 7.2E7 kilogram

Milk: $1.2 \mathrm{Eg}$ liter

Meat: 1.0E8 kilogram

0.5

0.5

Meteorology Period of Record: 19 APR $67-18$ APR 69 Recovery: 83\%

SITE SPECIFIC DATA - WATERBORNE PATHWAYS

Average Dilution Flow from

Plant: $573 \mathrm{ft} 3 / \mathrm{s}$

Drinking Water:

Exposed Population: 190,000

Dilution Factor: $2.6 \mathrm{E}-3(\mathrm{a})$

Fish:

Edible Harvest: $6.7 \mathrm{E} 4(\mathrm{~b}) \mathrm{kg} / \mathrm{yr}$

Dilution Factor: $0.013(\mathrm{c})$

(a) Drinking water dilution factor estimated by averaging dilution factor derived from FES (1972), suitably weighted for population.

(b) Includes both sport and commercial fish catch (FES, 1972).

(c) Dilution factor estimated by averaging sport and commercial dilution factor derived from FES (1972). 
POPULATION DOSE-COMMITMENT ESTIMATES AND AVERAGE INDIVIDUAL DOSE-COMMITMENT HISTOGRAM FOR POINT BEACH 1 AND 2

\section{Dose Commitments (person-rem) from Liquid Pathways}

Total Body GI-LLI $\underline{\text { Thyroid }}$ Bone Liver

Infant

9.7E-04

9.6E-04

$2.5 \mathrm{E}-03$

1.1E-04

$1.1 \mathrm{E}-03$

Child

1.2E -02

1.1E-02

2.2E-02

$5.0 \mathrm{E}-03$

$1.6 \mathrm{E}-02$

Teen

Adult

5. $6 \mathrm{E}-03$

4. 3E-03

7. $5 \mathrm{E}-03$

2. $6 \mathrm{E}-03$

7. $8 \mathrm{E}-03$

5.1E-02

3. $7 \mathrm{E}-02$

5. $8 \mathrm{E}-02$

1. $6 \mathrm{E}-02$

5.7E-02

TOTAL

6. $9 \mathrm{E}-02$

5.3E-02

9. $0 \mathrm{E}-02$

2. $3 \mathrm{E}-02$

8.1E-02

Dose Commitments (person-rem) from Airborne Pathways

\section{Total Body GI-LLI Thyroid Bone Liver Lung}

Infant

Child

Teen

Adult

TOTAL

\section{$6.9 \mathrm{E}-04$}

9. $9 \mathrm{E}-03$

$6.2 \mathrm{E}-03$

3.5E-02

$5.2 \mathrm{E}-02$
$6.9 \mathrm{E}-04$

9. $9 \mathrm{E}-03$

$6.3 \mathrm{E}-03$

3. $6 \mathrm{E}-02$

5. $3 \mathrm{E}-02$
2.7 -03

2. $4 \mathrm{E}-02$

2. $5 \mathrm{E}-04$

2. $8 \mathrm{E}-03$

$1.2 \mathrm{E}-02$

5.5E-02

2. $0 \mathrm{E}-03$

1.2E-02

9.3E-02

1.7E-02

7.0E-04

7.1E-04

9. $9 \mathrm{E}-03$

$1.0 \mathrm{E}-02$

$6.2 \mathrm{E}-03$

3. $5 \mathrm{E}-02$

6. $6 \mathrm{E}-03$

3. $6 \mathrm{E}-02$

$5.2 \mathrm{E}-02$

5. $4 \mathrm{E}-02$

Production/Consumption factors:

Produce: <1

Milk: 7.5

Meat: 1.1

FRACTION OF POPULATION RECEIVING AN INDICATED AVERAGE TOTAL-BODY DOSE COMMITMENT FROM AIRBORNE PATHWAYS

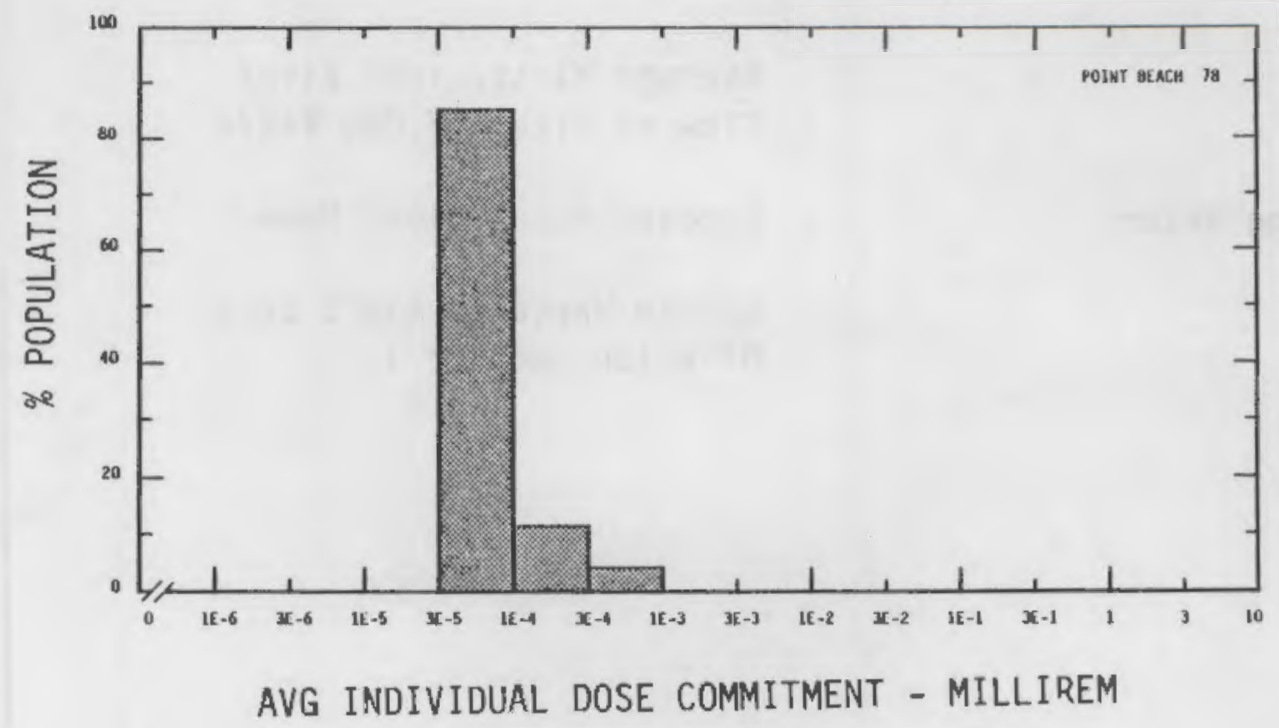




Location: $\quad$ N $44.6217^{\circ} \quad$ W $92.6330^{\circ}$

POPULATION DATA

Total Population Within 20-to-80-km Region: 2.2E6

Major Metropolitan Centers Within Region:

\begin{tabular}{|c|c|c|c|}
\hline Center & Population & Locat & \\
\hline Minneapolis-St. Paul SMSA & 2.1E6 & $50 \mathrm{~km}$ & NW \\
\hline Rochester & 57,000 & $64 \mathrm{~km}$ & SSE \\
\hline Hastings & 13,000 & $19 \mathrm{~km}$ & NW \\
\hline
\end{tabular}

SITE SPECIFIC DATA - AIRBORNE PATHWAYS

Average Annual State Production

of Crops and Animal Products

In $80-\mathrm{km}$ Radius Circle

Regional Productivity Factor:

Animal Grazing Factor:

Meteorology Period of Record: 22 MAR $74-21$ MAR 75 Recovery: $65 \%$
Veg: $1.2 \mathrm{E} 8 \mathrm{kilogram}$ Milk: $4.0 E 8$ liter Meat: $1.0 \mathrm{E} 8 \mathrm{kilogram}$

1

0.5

SITE SPECIFIC DATA - WATERBORNE PATHWAYS

$\begin{array}{ll} & \begin{array}{l}\text { Average Mississippi River } \\ \text { Flow at Site: } 15,000 \mathrm{ft}^{3} / \mathrm{s}\end{array} \\ \text { Drinking Water: } & \text { Exposed Population: None } \\ \text { Fish: } & \text { Edible Harvest: } 6.8 \mathrm{E} 5 \mathrm{~kg} / \mathrm{yr} \\ & \text { Dilution Factor: } 1\end{array}$


POPULATION DOSE-COMMITMENT ESTIMATES ANO

AVERAGE INOIVIDUAL OOSE-COMMITMENT HISTOGRAM FOR

PRAIRIE ISLANO 1 AND 2

Dose Commitments (person-rem) from Liquid Pathways

Total Body

$\underline{\text { GI-LLI }}$

Thyroid

Bone

Liver

Infant

$0.0 \mathrm{E}+00$

$0.0 \mathrm{E}+00$

$0.0 \mathrm{E}+00$

$0.0 \mathrm{E}+00$

$0.0 \mathrm{E}+00$

Child

1. $2 \mathrm{E}-02$

1.1E-02

1. $6 \mathrm{E}-03$

4. $0 \mathrm{E}-02$

4. 3E-02

Teen

Adult

1.8E-02

2.2E-02

$1.2 \mathrm{E}-03$

2. $6 \mathrm{E}-02$

3. $8 \mathrm{E}-02$

1. $7 \mathrm{E}-01$

1. $9 \mathrm{E}-01$

8. $4 \mathrm{E}-03$

1. $6 \mathrm{E}-01$

2. $3 \mathrm{E}-01$

TOTAL

2. $0 \mathrm{E}-01$

2. $2 \mathrm{E}-01$

1.1E-02

2. $2 \mathrm{E}-01$

3.1E-01

Dose Commitments (person-rem) from Airborne Pathways

Total Body GI-LLI Thyroid Bone Liver Lung

Infant
Child
Teen
Adult
TOTAL

3. $6 \mathrm{E}-03$

3. $6 \mathrm{E}-03$

4.9E-03

$2.8 \mathrm{~F}-03$

3. $6 \mathrm{E}-03$

4. $1 \mathrm{E}-03$

3. $\mathrm{E}-02$

4. $4 \mathrm{E}-02$

5. 3E-02

3.1E-02

4. $4 \mathrm{E}-02$

5.2E-02

3.1E-02

3.1E-02

3. $4 \mathrm{E}-02$

2. $3 \mathrm{E}-02$

3. 1E-02

4.1E-02

2. $6 \mathrm{E}-01$

1. $8 \mathrm{E}-01$

1. $9 \mathrm{E}-01$

1. $4 \mathrm{E}-01$

1. $8 \mathrm{E}-01$

2.1E-01

2. $6 \mathrm{E}-01$

2. $8 \mathrm{E}-01$

1. $9 E-01$

2. $6 \mathrm{E}-01$

3.1E-01

\section{Production/Consumption factors:}

Produce: $<1$

Milk: 1.4

Meat: $<1$

FRACTION OF POPULATION RECEIVING AN INOICATED AVERAGE TOTAL-BODY DOSE COMMITMENT FROM AIRBORNE PATHWAYS

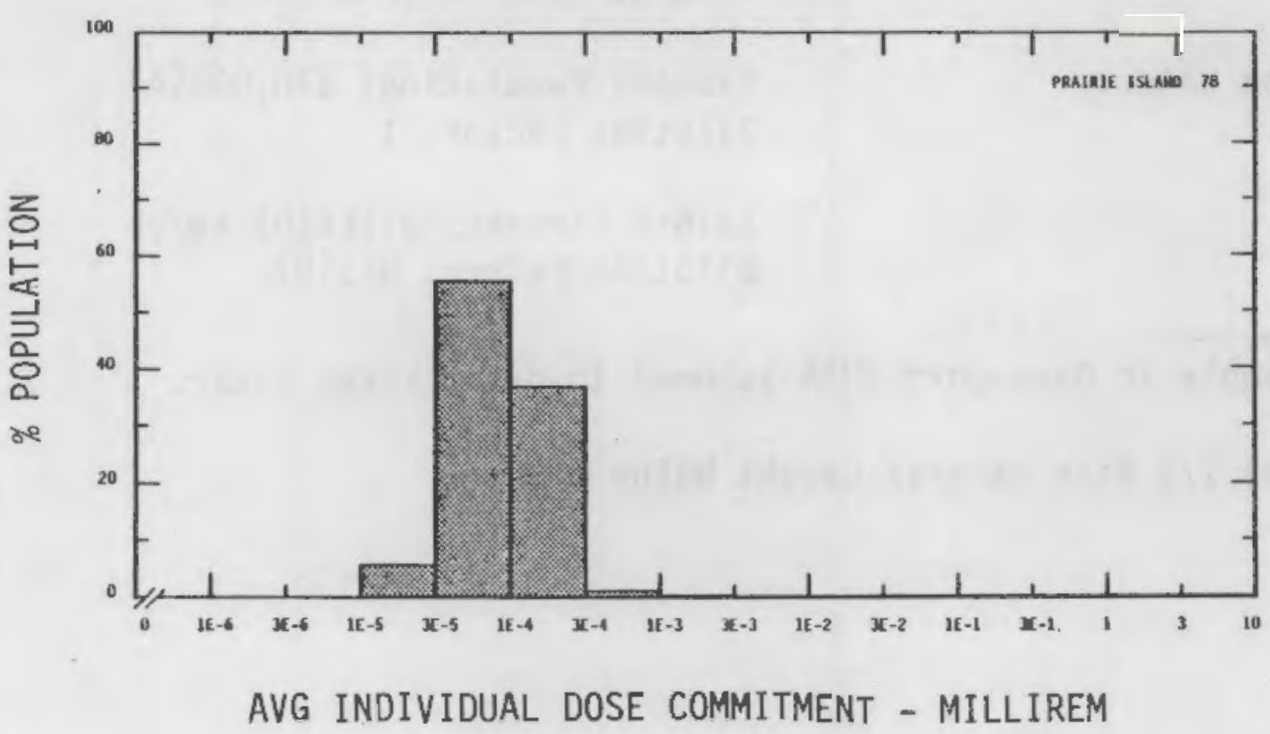


Site: QUAD CITIES

ROCK ISLAND, ILLINOIS

Location: N 41.72720 W 90.34170

POPULATION DATA

Total Population Within 20-to-80-km Region: 6.7E5

Major Metropolitan Centers Within Region:

Center

Population

Location

Davenport SMSA

370,000

$40 \mathrm{~km} \quad \mathrm{SW}$

SITE SPECIFIC DATA - AIRBORNE PATHWAYS

Average Annual State Production

of Crops and Animal Products

In 80-km Radius Circle

Regional Productivity Factor:

Animal Grazing Factor:

Meteorology Period of Record: 1 JAN $74-31$ DEC 75 Recovery: $88 \%$
Veg: $1.1 \mathrm{E} 8 \mathrm{kilogram}$

Milk: $1.8 \mathrm{E} 8$ liter

Meat: $1.9 \mathrm{E} 8$ kilogram

1

0.5

SITE SPECIFIC DATA - WATERBORNE PATHWAYS

Average Mississippi River

Flow at Site: $47,000 \mathrm{ft}^{3} / \mathrm{s}$

Drinking Water:

Exposed Population: $370,000(a)$

Dilution Factor: 1

Fish:

Edible Harvest: $2.1 \mathrm{E} 6$ (b) $\mathrm{kg} / \mathrm{yr}$ Dilution Factor: $0.5(\mathrm{~b})$

(a) All people in Oavenport SMSA assumed to drink river water.

(b) Assumes $1 / 2$ fish harvest caught below plant. 
POPULATION DOSE-COMMITMENT ESTIMATES AND

AVERAGE INDIVIDUAL DOSE-COMMITMENT HISTOGRAM FOR

QUAD CITIES 1 AND 2

Dose Commi tments (person-rem) from Liquid Pathways

Total Body GI-LLI Thyroid Bone Liver

$\begin{array}{llllll}\text { Infant } & 2.0 \mathrm{E}-03 & 4.2 \mathrm{E}-04 & 1.4 \mathrm{E}-03 & 1.4 \mathrm{E}-02 & 1.8 \mathrm{E}-02 \\ \text { Child } & 2.7 \mathrm{E}-01 & 1.5 \mathrm{E}-02 & 1.1 \mathrm{E}-02 & 1.3 \mathrm{E}+00 & 1.5 \mathrm{E}+00 \\ \text { Teen } & 4.8 \mathrm{E}-01 & 2.2 \mathrm{E}-02 & 3.6 \mathrm{E}-03 & 7.2 \mathrm{E}-01 & 1.2 \mathrm{E}+00 \\ \text { Adult } & 5.2 \mathrm{E}+00 & 1.8 \mathrm{E}-01 & 2.6 \mathrm{E}-02 & 4.2 \mathrm{E}+00 & 7.1 \mathrm{E}+00 \\ \text { TOTAL } & 6.0 \mathrm{E}+00 & 2.2 \mathrm{E}-01 & 4.2 \mathrm{E}-02 & 6.2 \mathrm{E}+00 & 9 . \mathrm{BE}+00\end{array}$

Dose Commi tments (person-rem) from Airborne Pathways

Total Body GI-LLI Thyroid Bone Liver Lung

$\begin{array}{lllllll}\text { Infant } & 1.8 \mathrm{E}-02 & 1.6 \mathrm{E}-02 & 1.7 \mathrm{E}+00 & 2.3 \mathrm{E}-02 & 2.5 \mathrm{E}-02 & 1.8 \mathrm{E}-02 \\ \text { Chi1d } & 2.2 \mathrm{E}-01 & 2.1 \mathrm{E}-01 & 1.8 \mathrm{E}+01 & 3.6 \mathrm{E}-01 & 2.8 \mathrm{E}-01 & 2.1 \mathrm{E}-01 \\ \text { Teen } & 1.5 \mathrm{E}-01 & 1.7 \mathrm{E}-01 & 9.4 \mathrm{E}+00 & 2.0 \mathrm{E}-01 & 1.9 \mathrm{E}-01 & 1.6 \mathrm{E}-01 \\ \text { Adult } & 8.8 \mathrm{E}-01 & 9.9 \mathrm{E}-01 & 4.0 \mathrm{E}+01 & 1.0 \mathrm{E}+00 & 1.0 \mathrm{E}+00 & \mathrm{~B} .8 \mathrm{E}-01 \\ \text { TOTAL } & 1.3 \mathrm{E}+00 & 1.4 \mathrm{E}+00 & 7.0 \mathrm{E}+01 & 1.6 \mathrm{E}+00 & 1.5 \mathrm{E}+00 & 1.3 \mathrm{E}+00\end{array}$

Production/Consumption factors:

Produce: <1 Milk: $2.0 \quad$ Meat: 3.5

FRACTION OF POPULATION RECEIVING AN INDICATEO AVERAGE TOTAL-BODY DOSE COMMITMENT FROM AIRBORNE PATHWAYS

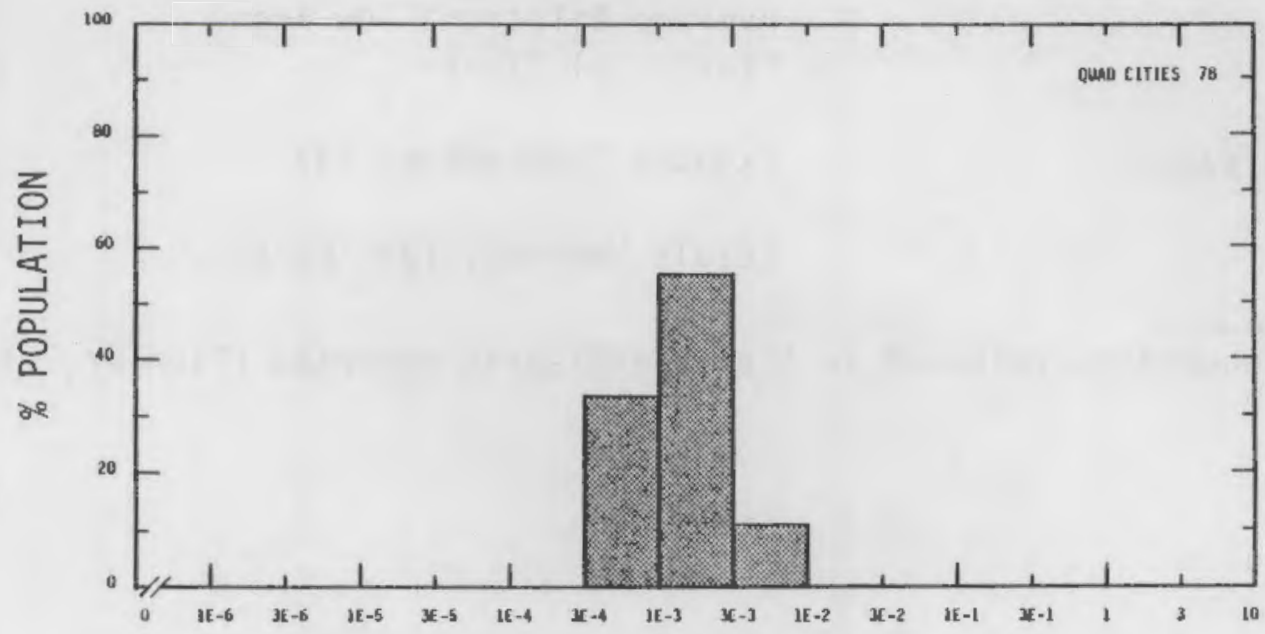

AVG INDIVIDUAL DOSE COMMITMENT - MILLIREM 
Site: RANCHO SECO

Location: N $38.3461^{\circ} \quad$ W $121.1186^{\circ}$

POPULATION DATA

Total Population Within 20-to-80-km Region: 1.5E6

Major Metropolitan Centers Within Region:

\begin{tabular}{llll}
\multicolumn{1}{c}{ Center } & Population & \multicolumn{2}{c}{ Location } \\
\cline { 2 - 4 } & 900,000 & $35 \mathrm{~km}$ & NNW \\
Sacramento SMSA & 120,000 & $45 \mathrm{~km}$ & SW \\
Stockton & & &
\end{tabular}

SITE SPECIFIC DATA - AIRBORNE PATHWAYS

Average Annual State Production

of Crops and Animal Products

In 80-km Radius Circle

Regional Productivity Factor:

Animal Grazing Factor:

Meteorology Period of Record: 1 FEB 75 - 31 JAN 76 Recovery: 98\%
Veg: $4.8 \mathrm{E} 7 \mathrm{kilogram}$

Milk: 2.3E8 liter

Meat: $5.0 \mathrm{E} 7 \mathrm{kilogram}$

1

0.9

SITE SPECIFIC DATA - WATERBORNE PATHWAYS

Average Dilution $\mathrm{Fl}$ ow from

Plant: (a) $\mathrm{ft}^{3} / \mathrm{s}$

Orinking Water:

Exposed Population: (a)

Fish:

Edible Harvest: (a) $\mathrm{kg} / \mathrm{yr}$

(a) No radionuclides released in liquid effluents reported (Tichler, et al., 1981). 
POPULATION DOSE-COMMITMENT ESTIMATES AND

AVERAGE INOIVIDUAL DOSE-COMMITMENT HISTOGRAM FOR

RANCHO SECO

Dose Commitments (person-rem) from Liquid Pathways

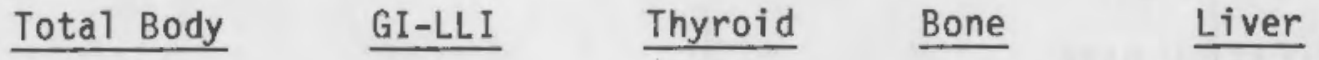

Infant

$0.0 \mathrm{E}+00$

$0.0 \mathrm{E}+00$

$0.0 \mathrm{E}+00$

$0.0 E+00$

$0.0 E+00$

Child

$0.0 \mathrm{E}+00$

$0.0 E+00$

$0.0 E+00$

$0.0 \mathrm{E}+00$

$0.0 E+00$

Teen

Adult

$0.0 \mathrm{E}+00$

$0.0 \mathrm{E}+00$

$0.0 E+00$

$0.0 E+00$

$0.0 E+00$

$0.0 \mathrm{E}+00$

$0.0 E+00$

$0.0 \mathrm{E}+00$

$0.0 \mathrm{E}+00$

$0.0 \mathrm{E}+00$

TOTAL

$0.0 \mathrm{E}+00$

$0.0 E+00$

$0.0 \mathrm{E}+00$

$0.0 E+00$

$0.0 E+00$

Dose Commitments (person-rem) from Airborne Pathways

\section{Total Body GI-LLI Thyroid Bone Liver Lung}

Infant

2.2E-03 2.2E-03

$5.6 \mathrm{E}-03$

8.3E-04

2.2E-03

2. $2 E-03$

Child

2. $8 \mathrm{E}-02$

2. $8 \mathrm{E}-02$

4.7E-02

1. $0 \mathrm{E}-02$

2. $8 \mathrm{E}-02$

2. $8 \mathrm{E}-02$

Teen

1. $8 \mathrm{E}-02$

1. $8 \mathrm{E}-02$

2. $5 E-02$

7. $0 \mathrm{E}-03$

1. $8 \mathrm{E}-02$

- 1.9E-02

Adult

1. $0 \mathrm{E}-01$

1. $0 \mathrm{E}-01$

1. $2 \mathrm{E}-01$

4.1E-02

1. $0 \mathrm{E}-01$

1. $0 \mathrm{E}-01$

TOTAL

1.5E-01

$1.5 \mathrm{E}-01$

2. $0 E-01$

$5.9 \mathrm{E}-02$

1.5E-01

1. $5 \mathrm{E}-01$

Production/Consumption factors:
Produce: <1
Milk: 1.2
Meat: $<1$

FRACTION OF POPULATION RECEIVING AN INDICATED AVERAGE TOTAL-BODY DOSE COMMITMENT FROM AIRBORNE PATHWAYS

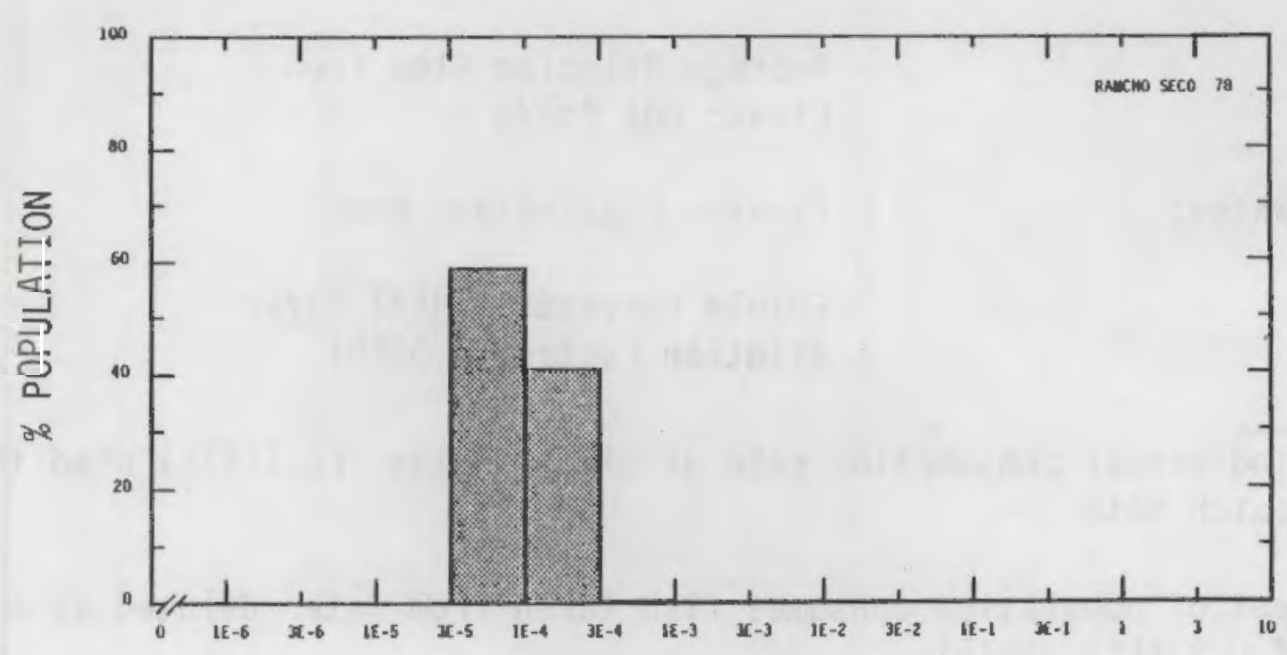

AVG INDIVIDUAL DOSE COMMITMENT - MILLIREM 
Total Population Within 20-to-80-km Region: $6.4 \mathrm{E5}$

\begin{tabular}{|c|c|c|c|}
\hline Center & Population & \multicolumn{2}{|c|}{ Location } \\
\hline Florence & 29,000 & $40 \mathrm{~km}$ & SE \\
\hline Sumter & 27,000 & $53 \mathrm{~km}$ & SSW \\
\hline
\end{tabular}

SITE SPECIFIC DATA - AIRBORNE PATHWAYS

Average Annual State Production

of Crops and Animal Products

In $80-\mathrm{km}$ Radius Circle

Regional Productivity Factor:

Animal Grazing Factor:

Meteorology Period of Record: 1 JAN 75 - 31 DEC 75 Recovery: 94\%
Veg: 7.4E6 kilogram

Milk: $5.7 \mathrm{E} 7$ liter

1

0.8
Meat: $5.0 \mathrm{E} 7 \mathrm{kilogram}$

SITE SPECIFIC DATA - WATERBORNE PATHWAYS

Average Dilution Flow from

Plant: $801 \mathrm{ft}^{3} / \mathrm{s}$

Drinking Water:

Exposed Population: None

Fish:

Edible Harvest: $1.8(\mathrm{a}) \mathrm{kg} / \mathrm{yr}$ Dilution Factor: 0.02 (b)

(a) Average individual consumption rate as given in the FES (1975) used in lieu of catch data.

(b) Ten percent of population consumes fish taken from water diluted by a factor of 0.2 (FES, 1975). 

AVERAGE INDIVIDUAL DOSE-COMMITMENT HISTOGRAM FOR H. B. ROBINSON

Dose Commi tments (person-rem) from Liquid Pathways Total Body

$$
\text { GI-LLI }
$$

Thyroid

Bone

Liver

$\begin{array}{llllll}\text { Infant } & 0.0 \mathrm{E}+00 & 0.0 \mathrm{E}+00 & 0.0 \mathrm{E}+00 & 0.0 \mathrm{E}+00 & 0.0 \mathrm{E}+00 \\ \text { Child } & 1.7 \mathrm{E}-02 & 1.0 \mathrm{E}-03 & 2.0 \mathrm{E}-03 & 7.6 \mathrm{E}-02 & 9.2 \mathrm{E}-02 \\ \text { Teen } & 3.2 \mathrm{E}-02 & 1.9 \mathrm{E}-03 & 1.4 \mathrm{E}-03 & 4.6 \mathrm{E}-02 & 7.9 \mathrm{E}-02 \\ \text { Adult } & 3.5 \mathrm{E}-01 & 1.6 \mathrm{E}-02 & 9.7 \mathrm{E}-03 & 2.7 \mathrm{E}-01 & 4.7 \mathrm{E}-01 \\ \text { TOTAL } & 4.0 \mathrm{E}-01 & 1.9 \mathrm{E}-02 & 1.3 \mathrm{E}-02 & 3.9 \mathrm{E}-01 & 6.4 \mathrm{E}-01\end{array}$

Dose Commitments (person-rem) from Airborne Pathways Total Body GI-LLI Thyroid Bone Liver Lung

$\begin{array}{lllllll}\text { Infant } & 6.8 \mathrm{E}-04 & 6.8 \mathrm{E}-04 & 1.0 \mathrm{E}-03 & 5.6 \mathrm{E}-04 & 6.8 \mathrm{E}-04 & 7.0 \mathrm{E}-04 \\ \text { Child } & 8.0 \mathrm{E}-03 & 8.0 \mathrm{E}-03 & 1.0 \mathrm{E}-02 & 6.2 \mathrm{E}-03 & 8.0 \mathrm{E}-03 & 8.3 \mathrm{E}-03 \\ \text { Teen } & 5.7 \mathrm{E}-03 & 5.7 \mathrm{E}-03 & 6.6 \mathrm{E}-03 & 4.5 \mathrm{E}-03 & 5.7 \mathrm{E}-03 & 6.2 \mathrm{E}-03 \\ \text { Adult } & 3.4 \mathrm{E}-02 & 3.4 \mathrm{E}-02 & 3.7 \mathrm{E}-02 & 2.7 \mathrm{E}-02 & 3.4 \mathrm{E}-02 & 3.6 \mathrm{E}-02 \\ \text { TOTAL } & 4.9 \mathrm{E}-02 & 4.9 \mathrm{E}-02 & 5.5 \mathrm{E}-02 & 3.9 \mathrm{E}-02 & 4.9 \mathrm{E}-02 & 5.1 \mathrm{E}-02\end{array}$

Production/Consumption factors:

Produce: $<1 \quad$ Milk: $<1 \quad$ Meat: $<1$

FRACTION OF POPULATION RECEIVING AN INDICATED AVERAGE TOTAL-BODY DOSE COMMITMENT FROM AIRBORNE PATHWAYS

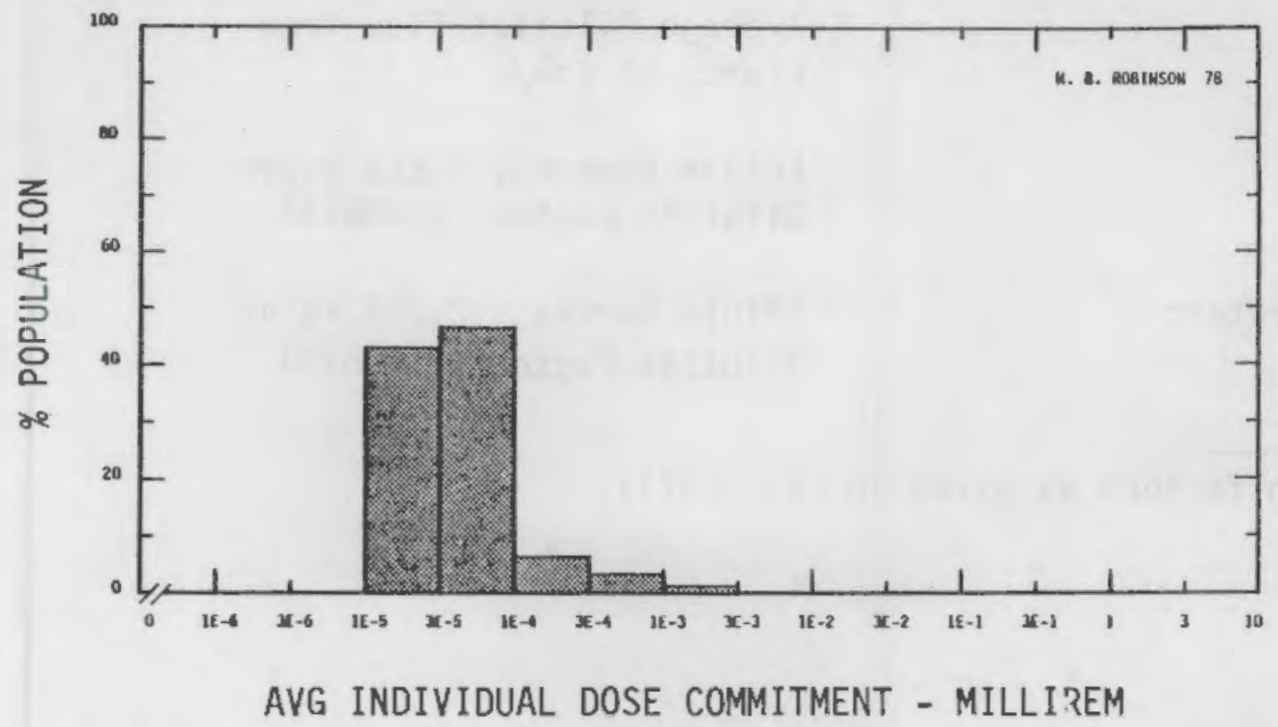


Site: ST. LUCIE

FORT PIERCE, FLORIDA

Location: $\quad$ N 27.44660 W 80.32660

POPULATION DATA

Total Population Within 20-to-80-km Region: 2.9E5

Major Metropolitan Centers Within Region:

Center Population Location

West Palm Beach

72,000

Ft. Pierce

37,000

$80 \mathrm{~km} \quad$ SSE

Vero Beach

15,000

$13 \mathrm{~km} \quad \mathrm{NW}$

$40 \mathrm{~km} \quad \mathrm{NNW}$

SITE SPECIFIC DATA - AIRBORNE PATHWAYS

Average Annual State Production

of Crops and Animal Products

In 80-km Radius Circle

Regional Productivity Factor:

Animal Grazing Factor:
Veg: $2.8 E 7$ kilogram

Milk: $1.1 \mathrm{E} 8$ liter

Meat: $7.2 E 7$ kilogram

0.5

1

Meteorology Period of Record: 1 JAN 76 - 31 DEC 76 Recovery: 92\%

SITE SPECIFIC DATA - WATERBORNE PATHWAYS

Average Dilution Flow from

Plant: $65 \mathrm{ft}^{3} / \mathrm{s}$

Fish:

Edible Harvest: $2.6 \mathrm{E} 5 \mathrm{~kg} / \mathrm{yr}$

Dilution Factor: $0.005(\mathrm{a})$

Invertebrates:

Edible Harvest: $2.7 E 4 \mathrm{~kg} / \mathrm{yr}$

Dilution Factor: 0.005 (a)

(a) Dilution factors as given in FES (1973). 
POPULATION DOSE-COMMITMENT ESTIMATES AND

AVERAGE INDIVIDUAL DOSE-COMMITMENT HISTOGRAM FOR

SAINT LUCIE

Dose Commitments (person-rem) from Liquid Pathways

$\underline{\text { Total Body GI-LLI } \underline{\text { Thyroid }} \text { Bone Liver }}$

$\begin{array}{llllll}\text { Infant } & 0.0 \mathrm{E}+00 & 0.0 \mathrm{E}+00 & 0.0 \mathrm{E}+00 & 0.0 \mathrm{E}+00 & 0.0 \mathrm{E}+00 \\ \text { Child } & 5.0 \mathrm{E}-03 & 1.2 \mathrm{E}-02 & 1.7 \mathrm{E}-02 & 9.3 \mathrm{E}-03 & 1.2 \mathrm{E}-02 \\ \text { Teen } & 5.3 \mathrm{E}-03 & 2.5 \mathrm{E}-02 & 1.2 \mathrm{E}-02 & 5.6 \mathrm{E}-03 & 1.1 \mathrm{E}-02 \\ \text { Adult } & 4.6 \mathrm{E}-02 & 2.2 \mathrm{E}-01 & 7.9 \mathrm{E}-01 & 3.3 \mathrm{E}-02 & 6.4 \mathrm{E}-02 \\ \text { TOTAL } & 5.6 \mathrm{E}-02 & 2.5 \mathrm{E}-01 & 1.1 \mathrm{E}-01 & 4.8 \mathrm{E}-02 & 8.7 \mathrm{E}-02\end{array}$

Dose Commitments (person-rem) from Airborne Pathways

Total Body GI-LLI Thyroid Bone Liver Lung

$\begin{array}{lllllll}\text { Infant } & 1.4 \mathrm{E}-02 & 1.4 \mathrm{E}-02 & 5.0 \mathrm{E}-02 & 1.3 \mathrm{E}-02 & 1.5 \mathrm{E}-02 & 1.5 \mathrm{E}-02 \\ \text { Child } & 1.7 \mathrm{E}-01 & 1.7 \mathrm{E}-01 & 4.1 \mathrm{E}-01 & 1.5 \mathrm{E}-01 & 1.7 \mathrm{E}-01 & 1.8 \mathrm{E}-01 \\ \text { Teen } & 1.2 \mathrm{E}-01 & 1.2 \mathrm{E}-01 & 2.3 \mathrm{E}-01 & 1.1 \mathrm{E}-01 & 1.2 \mathrm{E}-01 & 1.3 \mathrm{E}-01 \\ \text { Adu1t } & 7.3 \mathrm{E}-01 & 7.3 \mathrm{E}-01 & 1.1 \mathrm{E}+00 & 6.6 \mathrm{E}-01 & 7.3 \mathrm{E}-01 & 7.7 \mathrm{E}-01 \\ \text { TOTAL } & 1.0 \mathrm{E}+00 & 1.0 \mathrm{E}+00 & 1.8 \mathrm{E}+00 & 9.3 \mathrm{E}-01 & 1.0 \mathrm{E}+00 & 1.1 \mathrm{E}+00\end{array}$

Production/Consumption factors:

Produce: <1 Milk: $1.5 \quad$ Meat: 1.6

FRACTION OF POPULATION RECEIVING AN INDICATED AVERAGE TOTAL-BODY DOSE COMMITMENT FROM AIRBORNE PATHWAYS

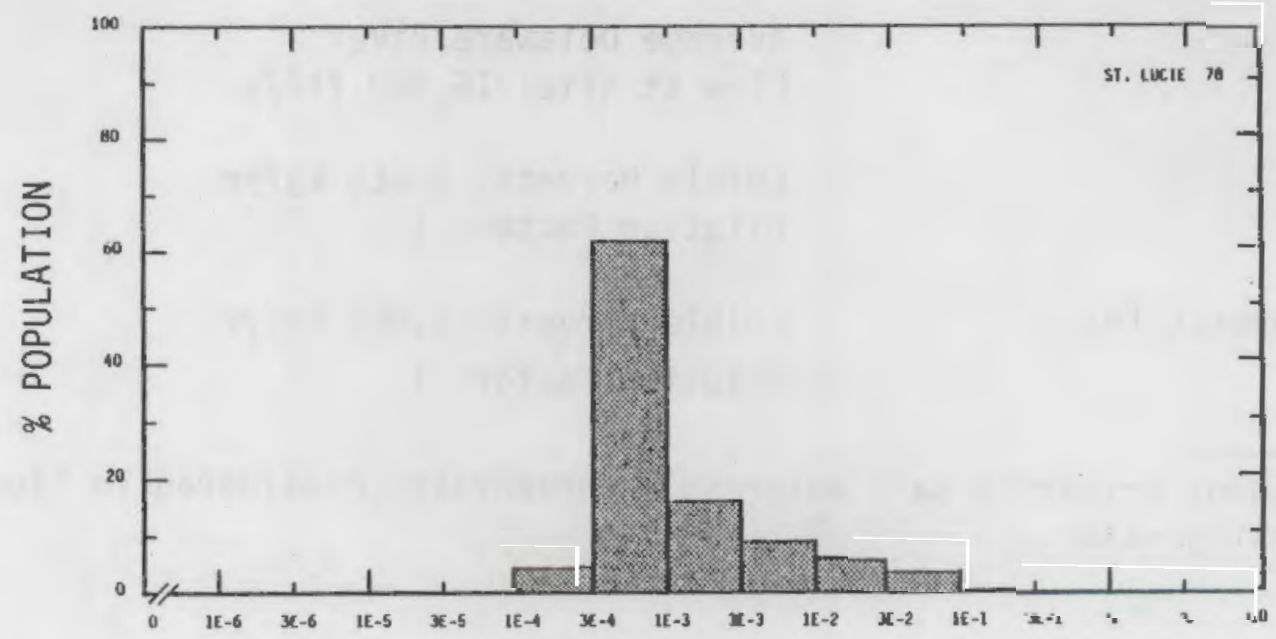

AVG INDIVIDUAL DOSE COMMITMENT - MILLIREM 


Location: $\quad N 39.46280$ W 75.53560

POPULATION DATA

Total Population Within 20-to-80-km Region: 4.9E6

Major Metropolitan Centers Within Region:

Center

Philadelphia SMSA

Wilmington SMSA

Chester
Population

$4,900,000$

500,000

57,000
Location

$64 \mathrm{~km} \quad \mathrm{NE}$

$29 \mathrm{~km} \quad \mathrm{~N}$

$45 \mathrm{~km} \quad \mathrm{NNE}$

SITE SPECIFIC DATA - AIRBORNE PATHWAYS

Average Annual State Production

of Crops and Animal Products

In 80-km Radius Circle

Regional Productivity Factor:

Animal Grazing Factor:
Veg: $7.4 \mathrm{E} 7 \mathrm{kilogram}$

Milk: $2.7 \mathrm{E} 8$ liter

Meat: $2.4 \mathrm{E} 7$ kilogram

0.9

0.6

Meteorology Period of Record: 1 JUN 70 - 31 MAY 71 Recovery: 95\%

Average Delaware River

Flow at Site: $16,000 \mathrm{ft}^{3} / \mathrm{s}$

Fish:

Invertebrates: (a)
Edible Harvest: $3.6 \mathrm{E} 5 \mathrm{~kg} / \mathrm{yr}$

Dilution Factor: 1

Edible Harvest: $1.6 E 5 \mathrm{~kg} / \mathrm{yr}$

Dilution Factor: 1

(a) Environment primarily salt water so invertebrates considered in lieu of drinking water. 
POPULATION DOSE-CDMMITMENT ESTIMATES AND

AVERAGE INDIVIDUAL DOSE-COMMITMENT HISTOGRAM FOR

SALEM

Dose Commitments (person-rem) from Liquid Pathways

Total Body

$$
\underline{G I-L L I}
$$

Thyroid

Bone

Liver

Infant

$0.0 \mathrm{E}+00$

$0.0 \mathrm{E}+00$

$0.0 \mathrm{E}+00$

$0.0 \mathrm{E}+00$

$0.0 \mathrm{E}+00$

Child

2.7E-02

5. 5E-02

$2.4 \mathrm{E}-03$

$1.0 \mathrm{E}-02$

2.6E-02

Teen

Adult

1.1E-01

$1.1 \mathrm{E}-01$

$1.7 \mathrm{E}-03$

$6.3 \mathrm{E}-03$

2. $3 \mathrm{E}-02$

TOTAL

1. $6 \mathrm{E}-01$

1. $0 \mathrm{E}+00$

1.2E-02

3. $9 \mathrm{E}-02$

1. $4 \mathrm{E}-01$

$1.2 \mathrm{E}+00$

$1.6 \mathrm{E}-02$

$5.5 \mathrm{E}-02$

1.9E-01

Dose Commitments (person-rem) from Airborne Pathways

\begin{tabular}{|c|c|c|c|c|c|}
\hline Total Body & GI-LLI & Thyroid & Bone & Liver & Lung \\
\hline $2.4 \mathrm{E}-03$ & $1.8 E-03$ & $2.7 \mathrm{E}-03$ & $3.8 \mathrm{E}-03$ & $7.9 \mathrm{E}-03$ & $2.5 E-03$ \\
\hline $3.4 E-02$ & $2.5 E-02$ & 3. $0 \mathrm{E}-02$ & $3.5 E-02$ & $7.1 E-02$ & $3.0 \mathrm{E}-02$ \\
\hline $2.7 E-02$ & 1. $7 \mathrm{E}-02$ & $1.9 \mathrm{E}-02$ & 1. $4 \mathrm{E}-02$ & $3.8 \mathrm{E}-02$ & $2.0 \mathrm{E}-02$ \\
\hline $1.5 \mathrm{E}-01$ & $9.8 \mathrm{E}-02$ & 1. $0 \mathrm{E}-01$ & $5.5 \mathrm{E}-02$ & $1.6 \mathrm{E}-01$ & 1. $0 \mathrm{E}-01$ \\
\hline 2. $1 E-01$ & $1.4 \mathrm{E}-01$ & $1.6 \mathrm{E}-01$ & 1.1E-01 & $2.8 \mathrm{E}-01$ & $1.6 \mathrm{E}-01$ \\
\hline
\end{tabular}

Production/Consumption factors:
Produce: $<1$
Milk: $<1$
Meat: <1

FRACTION OF POPULATION RECEIVING AN INDICATED AVERAGE TOTAL-BODY DOSE COMMITMENT FROM AIRBORNE PATHWAYS

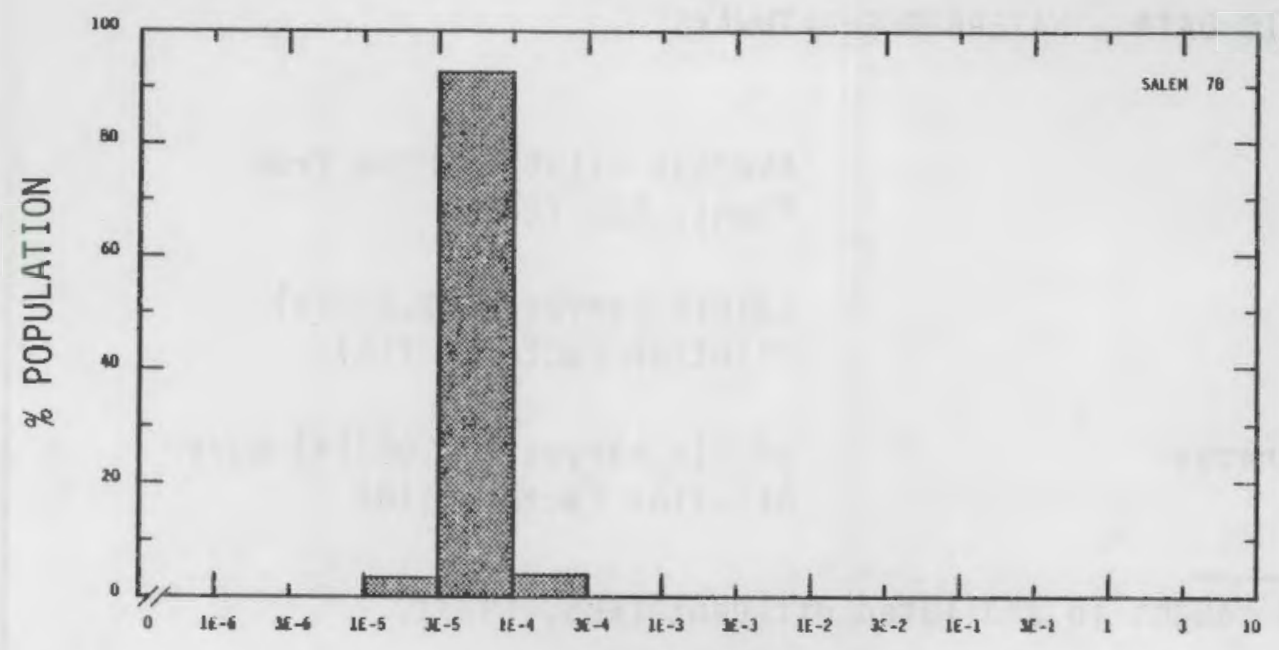

AVG INDIVIDUAL DOSE COMMITMENT - MILLIREM 
Location: N 33.36860 W 117.55440

POPULATION DATA

Total Population Within 20-to-80-km Region: 3.9 E6

Major Metropolitan Centers Within Region:

\begin{tabular}{|c|c|c|c|}
\hline Center & Population & \multicolumn{2}{|c|}{ Location } \\
\hline San Diego SMSA & $1,500,000$ & $68 \mathrm{~km}$ & SSE \\
\hline Riverside & 160,000 & $69 \mathrm{~km}$ & NE \\
\hline Anaheim & 190,000 & $69 \mathrm{~km}$ & NNW \\
\hline Santa Ana & 170,000 & $55 \mathrm{~km}$ & NNW \\
\hline
\end{tabular}

SITE SPECIFIC DATA - AIRBORNE PATHWAYS

Average Annual State Production

of Crops and Animal Products

In $80-\mathrm{km}$ Radius Circle

Regional Productivity Factor:

Animal Grazing Factor:
Veg: $4.8 E 7$ kilogram

Milk: 2.3E8 liter

Meat: $5.0 E 7$ kilogram

0.6

1

Meteorology Period of Record: 25 JAN $73-24$ JAN 76 Recovery: $88 \%$

SITE SPECIFIC DATA - WATERBORNE PATHWAYS

Average Dilution $\mathrm{Fl}$ ow from

Plant: $533 \mathrm{ft}^{3} / \mathrm{s}$

Fish:

Edible Harvest: $2.9 E 4(a)$

Dilution Factor: 1(a)

Invertebrates:

Edible Harvest: $2.9 \mathrm{E} 3(\mathrm{a}) \mathrm{kg} / \mathrm{yr}$

Dilution Factor: 1 (a)

(a) Seafood caught in undiluted effluent (FES, 1973). 
POPULATION DOSE-COMMITMENT ESTIMATES AND AVERAGE INOIVIDUAL DOSE-COMMITMENT HISTOGRAM FOR

SAN ONOFRE

Oose Commitments (person-rem) from Liquid Pathways Total Body GI-LLI Thyroid Bone Liver

$\begin{array}{llllll}\text { Infant } & 0.0 E+00 & 0.0 E+00 & 0.0 E+00 & 0.0 E+00 & 0.0 E+00 \\ \text { Child } & 8.3 E-02 & 1.2 E-01 & 2.7 E-02 & 3.2 E-01 & 3.9 E-01 \\ \text { Teen } & 1.4 E-01 & 2.5 E-01 & 2.0 E-02 & 1.9 E-01 & 3.3 E-01 \\ \text { Adult } & 1.5 E+00 & 2.2 E+00 & 1.3 E-01 & 1.1 E+00 & 2.0 E+00 \\ \text { TOTAL } & 1.7 E+00 & 2.6 E+00 & 1.8 E-01 & 1.6 E+00 & 2.7 E+00\end{array}$

Dose Commitments (person-rem) from Airborne Pathways

Total Body GI-LLI Thyroid Bone Liver Lung

$\begin{array}{lllllll}\text { Infant } & 6.7 \mathrm{E}-04 & 6.7 \mathrm{E}-04 & 9.3 \mathrm{E}-04 & 4.4 \mathrm{E}-04 & 7.2 \mathrm{E}-04 & 7.5 \mathrm{E}-04 \\ \text { Child } & 8.5 \mathrm{E}-03 & 8.6 \mathrm{E}-03 & 9.9 \mathrm{E}-03 & 4.8 \mathrm{E}-03 & 8.8 \mathrm{E}-03 & 9.6 \mathrm{E}-03 \\ \text { Teen } & 6.2 \mathrm{E}-03 & 6.3 \mathrm{E}-03 & 6.6 \mathrm{E}-03 & 3.4 \mathrm{E}-03 & 6.2 \mathrm{E}-03 & 7.4 \mathrm{E}-03 \\ \text { Aduit } & 3.6 \mathrm{E}-02 & 3.7 \mathrm{E}-02 & 3.7 \mathrm{E}-02 & 2.0 \mathrm{E}-02 & 3.6 \mathrm{E}-02 & 4.0 \mathrm{E}-02 \\ \text { TOTAL } & 5.1 \mathrm{E}-02 & 5.3 \mathrm{E}-02 & 5.5 \mathrm{E}-02 & 2.9 \mathrm{E}-02 & 5.2 \mathrm{E}-02 & 5.8 \mathrm{E}-02\end{array}$

Production/Consumption factors:

Produce: $<1 \quad$ Milk: $<1 \quad$ Meat: <1

FRACTION OF POPULATION RECEIVING AN INDICATED AVERAGE TOTAL-BODY DOSE COMMITMENT FROM AIRBORNE PATHWAYS

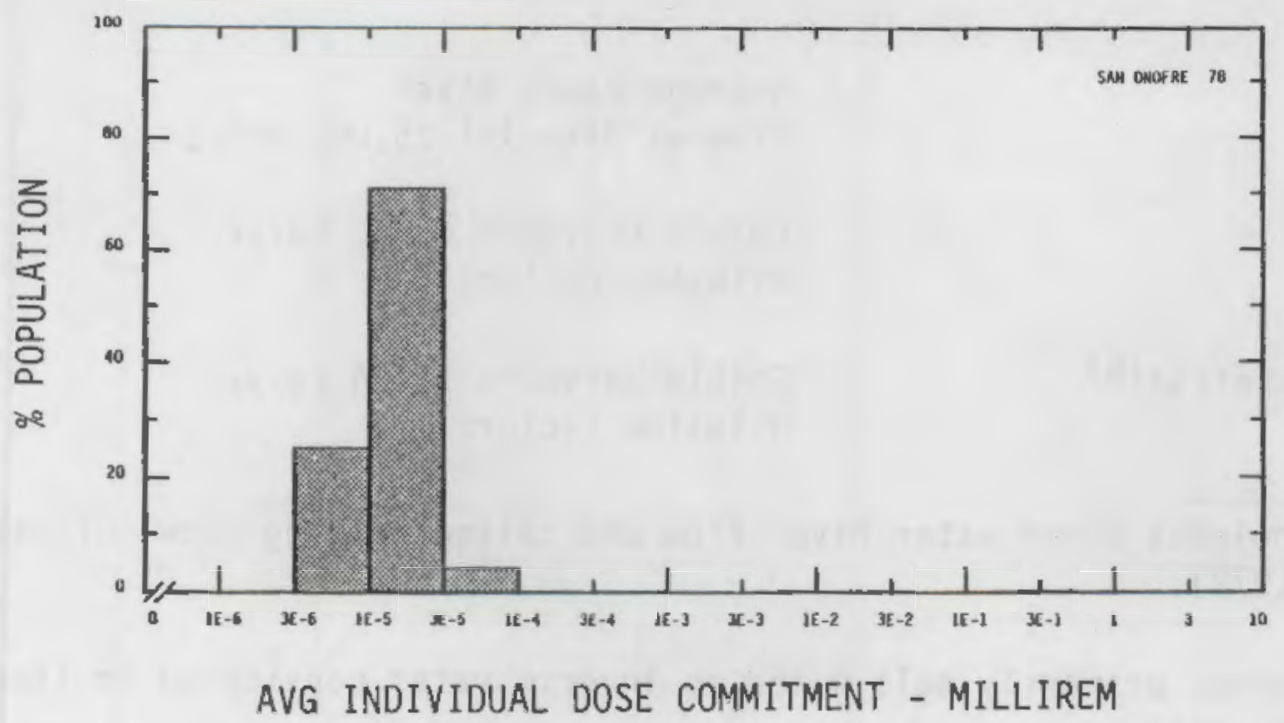


Location: N $37.16640 \quad$ W 76.69720

POPULATION DATA

Total Population Within 20-to-80-km Region: $1.8 \mathrm{E} 6$

Major Metropolitan Centers Within Region:

Center Population Location

Newport NewS SMSA $\quad 370,000 \quad 16 \mathrm{~km} \quad$ ESE

Norfold SMSA $\quad 810,000 \quad 50 \mathrm{~km} \quad$ SE

$\begin{array}{llll}\text { Richmond SMSA } & 600,000 & 78 \mathrm{~km} & \text { NW }\end{array}$

SITE SPECIFIC DATA - AIRBORNE PATHWAYS

Average Annual State Production

of Crops and Animal Products

In 80-km Radius Circle

Regional Productivity Factor:

Animal Grazing Factor:
Veg: $3.5 \mathrm{E} 7 \mathrm{kilogram}$

Milk: $1.5 \mathrm{E} 8$ liter

Meat: $7.4 \mathrm{E} 7$ kilogram

0.8

0.7

Meteorology Period of Record: 3 MAR $74-2$ MAR 75 Recovery: 91\%

SITE SPECIFIC DATA - WATERBORNE PATHWAYS

Average James River

Flow at Site: (a) $25,000 \mathrm{ft}^{3} / \mathrm{s}$

Fish:

Edible Harvest: $6.0 \mathrm{E} 5 \mathrm{~kg} / \mathrm{yr}$

Dilution Factor: 1

Invertebrates: (b)

Edible Harvest: $1.1 \mathrm{E} 6 \mathrm{~kg} / \mathrm{yr}$ Dilution Factor: 1

(a) Flow includes fresh water river flow and saline "mixing flow" of estuary (FES, 1972).

(b) Environment primarily salt water so invertebrates considered in lieu of drinking water. 
POPULATION DOSE-COMMITMENT ESTIMATES AND

AVERAGE INDIVIDUAL DOSE-COMMITMENT HISTOGRAM FOR

SURRY 1 AND 2

Dose Commitments (person-rem) from Liquid Pathways

Total Body GI-LLI Thyroid Bone Liver

Infant

Child

Teen

$0.0 \mathrm{E}+00$

$0.0 \mathrm{E}+00$

$0.0 E+00$

$0.0 E+00$

$0.0 \mathrm{E}+00$

1. $4 \mathrm{E}-01$

2.7E-01

4. 5E-01

9.1E-02

1. 5E-01

Adult

1.2E-01

5. $6 \mathrm{E}-01$

3.1E-01

5. 3E-02

1. $2 \mathrm{E}-01$

8. $5 \mathrm{E}-01$

$5.0 \mathrm{E}+00$

2. $0 E+00$

3. $1 E-01$

7. $4 \mathrm{E}-01$

TOTAL

$1.1 \mathrm{E}+00$

$5.8 \mathrm{E}+00$

$2.8 \mathrm{E}+00$

4. 5E-01

1. $0 \mathrm{E}+00$

Dose Commitments (person-rem) from Airborne Pathways Total Body GI-LLI Thyroid Bone Liver Lung

Infant

4. $2 E-03$

4. $0 \mathrm{E}-03$

4. $4 \mathrm{E}-02$

4.2E-03

6.1E-03

4. $5 E-03$

Child

5. 4E-02

5.2E-02

3. $0 \mathrm{E}-01$

4.2E-02

6. $6 \mathrm{E}-02$

5.7E-02

Teen

3. $9 \mathrm{E}-02$

3. $6 \mathrm{E}-02$

1. $4 \mathrm{E}-01$

2.5E-02

4. $3 \mathrm{E}-02$

4.2E-02

Adult

2. $3 \mathrm{E}-01$

2.1E-01

$5.9 E-01$

1. $3 \mathrm{E}-01$

2. $3 \mathrm{E}-01$

2. $3 \mathrm{E}-01$

TOTAL

3. $2 \mathrm{E}-01$

3.1E-01

$1.1 E+00$

2. $0 \mathrm{E}-01$

3. $5 \mathrm{E}-01$

3. $3 \mathrm{E}-01$

Production/Consumption factors:

Produce: $<1$

Milk: $<1$

Meat: <1

FRACTION OF POPULATION RECEIVING AN INDICATED AVERAGE TOTAL-BODY DOSE COMMITMENT FROM AIRBORNE PATHWAYS

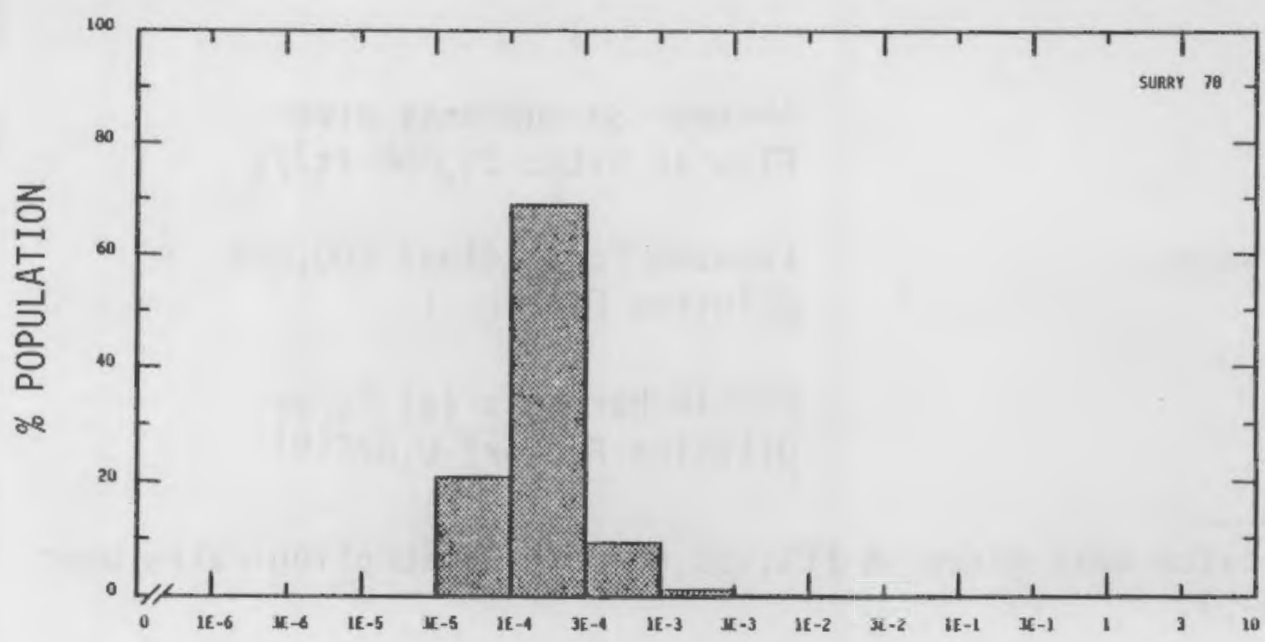

AVG INDIVIDUAL DOSE COMMITMENT - MILLIREM 
Location: N 40.15330 W 76.72690

POPULATION DATA

Total Population Within 20-to-80-km Region: $1.8 \mathrm{E} 6$

Major Metropolitan Centers Within Region:

\begin{tabular}{lllll}
\multicolumn{1}{c}{ Center } & Population & \multicolumn{2}{c}{ Location } \\
\cline { 2 - 3 } & 320,000 & $40 \mathrm{~km}$ & ESE \\
Lancaster SMSA & 410,000 & $16 \mathrm{~km}$ & NW \\
Harrisburg SMSA & 290,000 & $71 \mathrm{~km}$ & ENE \\
Reading SMSA & 330,000 & $23 \mathrm{~km}$ & S
\end{tabular}

SITE SPECIFIC DATA - AIRBORNE PATHWAYS

Average Annual State Production

of Crops and Animal Products

In $80-\mathrm{km}$ Radius Circle

Regional Productivity Factor:

Animal Grazing Factor:

Meteorology Period of Record: 1 OCT 72 - 30 SEP 73 Recovery: $80 \%$
Veg: $5.3 E 7 \mathrm{kilogram}$

Milk: 5.3E8 liter

Meat: $5.4 \mathrm{E} 7 \mathrm{kilogram}$

1

0.5

SITE SPECIFIC DATA - WATERBORNE PATHWAYS

Average Susquehanna River

Flow at Site: $34,000 \mathrm{ft}^{3} / \mathrm{s}$

Drinking Water:

Exposed Population: 200,000

Dilution Factor: 1

Fish:

Edible Harvest: (a) $\mathrm{kg} / \mathrm{yr}$

Dilution Factor: $0.025(\mathrm{~b})$

(a) No fish catch data given in FES, so generic consumption rates used (Table A-1).

(b) Ten percent of population consumes $25 \%$ of their fish from river (FES, 1972). 
POPULATION DOSE-COMMITMENT ESTIMATES AND

AVERAGE INDIVIDUAL DOSE-COMMITMENT HISTOGRAM FOR

THREE MILE ISLAND 1 ANO 2

Dose Commitments (person-rem) from Liquid Pathways

Total Body GI-LLI Thyroid Bone Liver

$\begin{array}{llllll}\text { Infant } & 1.2 \mathrm{E}-03 & 8.8 \mathrm{E}-04 & 5.9 \mathrm{E}-03 & 2.6 \mathrm{E}-03 & 4.3 \mathrm{E}-03 \\ \text { Child } & 3.7 \mathrm{E}-02 & 1.3 \mathrm{E}-02 & 4.5 \mathrm{E}-02 & 1.3 \mathrm{E}-01 & 1.6 \mathrm{E}-01 \\ \text { Teen } & 5.0 \mathrm{E}-02 & 9.7 \mathrm{E}-03 & 1.5 \mathrm{E}-02 & 6.8 \mathrm{E}-02 & 1.2 \mathrm{E}-01 \\ \text { Adu1t } & 5.4 \mathrm{E}-01 & 8.5 \mathrm{E}-02 & 1.1 \mathrm{E}-01 & 4.0 \mathrm{E}-01 & 7.2 \mathrm{E}-01 \\ \text { TOTAL } & 6.3 \mathrm{E}-01 & 1.1 \mathrm{E}-01 & 1.7 \mathrm{E}-01 & 6.0 \mathrm{E}-01 & 1.0 \mathrm{E}+00\end{array}$

Dose Commitments (person-rem) from Airborne Pathways

Total Body GI-LLI Thyroid Bone Liver Lung

$\begin{array}{lllllll}\text { Infant } & 2.2 \mathrm{E}-02 & 2.1 \mathrm{E}-02 & 4.9 \mathrm{E}-02 & 2.6 \mathrm{E}-02 & 3.2 \mathrm{E}-02 & 2.4 \mathrm{E}-02 \\ \text { Child } & 2.6 \mathrm{E}-01 & 2.4 \mathrm{E}-01 & 4.3 \mathrm{E}-01 & 2.6 \mathrm{E}-01 & 3.3 \mathrm{E}-01 & 2.8 \mathrm{E}-01 \\ \text { Teen } & 1.9 \mathrm{E}-01 & 1.8 \mathrm{E}-01 & 2.6 \mathrm{E}-01 & 1.6 \mathrm{E}-01 & 2.1 \mathrm{E}-01 & 2.1 \mathrm{E}-01 \\ \text { Aduit } & 1.1 \mathrm{E}+00 & 1.0 \mathrm{E}+00 & 1.3 \mathrm{E}+00 & 8.9 \mathrm{E}-01 & 1.1 \mathrm{E}+00 & 1.1 \mathrm{E}+00 \\ \text { TOTAL } & 1.6 \mathrm{E}+00 & 1.5 \mathrm{E}+00 & 2.1 \mathrm{E}+00 & 1.3 \mathrm{E}+00 & 1.7 \mathrm{E}+00 & 1.7 \mathrm{E}+00\end{array}$

Production/Consumption factors:

Produce: <1 Milk: $2.2 \quad$ Meat: <1

FRACTION OF POPULATION RECEIVING AN INDICATED AVERAGE TOTAL-BODY DOSE COMMITMENT FROM AIRBORNE PATHWAYS

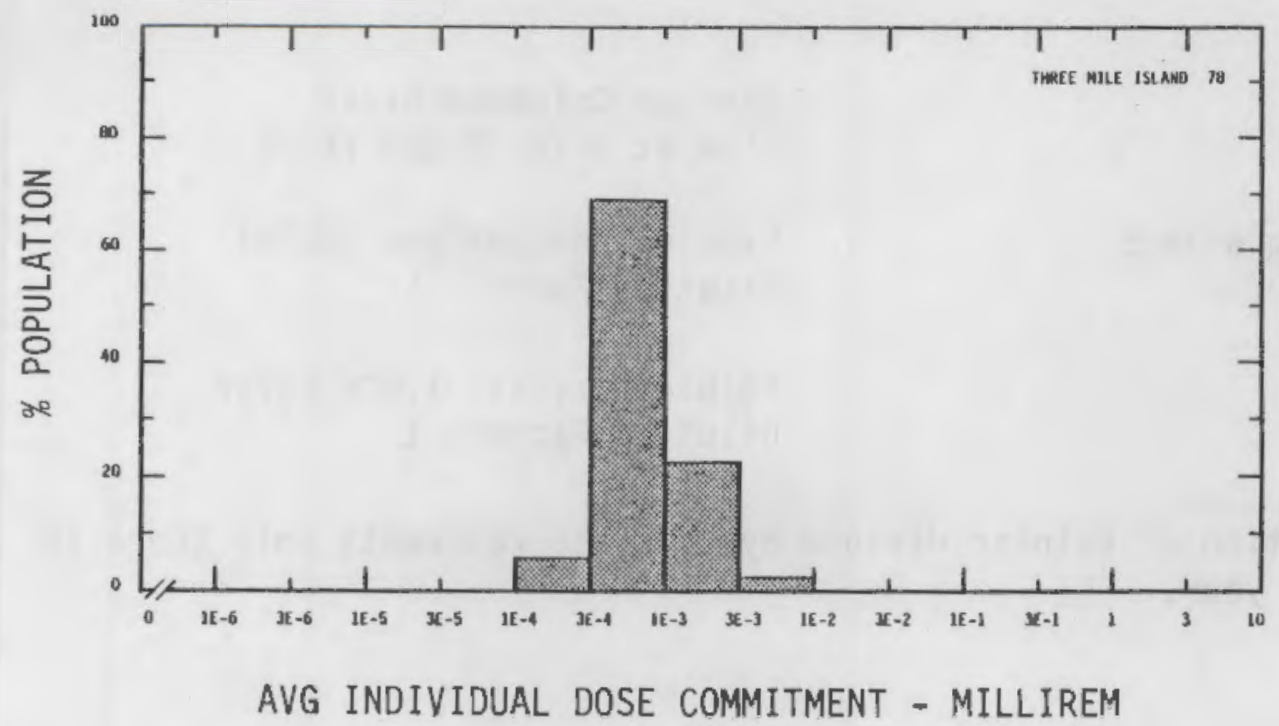


Site: TROJAN

PRESCOTT, OREGON

Location:

N 46.03720

W $\quad 122.88470$

POPULATION DATA

Total Population Within 20-to-80-km Region: 1.3E6

Major Metropolitan Centers Within Region:

Center

Portland SMSA

Longview

Astoria
Population

$1.2 \mathrm{E} 6$

33,000

12,000
Location

$63 \mathrm{~km} \quad$ SSE

$14 \mathrm{~km} \quad \mathrm{NNW}$

$72 \mathrm{~km} \quad \mathrm{WNW}$

SITE SPECIFIC DATA - AIRBORNE PATHWAYS

Average Annual State Production

of Crops and Animal Products

In 80-km Radius Circle

Regional Productivity Factor:

Animal Grazing Factor:
Veg: 6.4E7 kilogram

Mi1k: 3.7E7 liter

Meat: $2.6 \mathrm{E} 7 \mathrm{kilogram}$

0.9

0.75

Meteorology Period of Record: 1 SEP 71 - 31 AUG 74 Recovery: 90\%

SITE SPECIFIC DATA - WATERBORNE PATHWAYS

Average Columbia River

Flow at Site: $2.3 \mathrm{E} 5 \mathrm{ft}^{3} / \mathrm{s}$

Drinking Water:

Exposed Population: $490(a)$

Dilution Factor: 1

Fish:

Edible Harvest: $1.0 \mathrm{E} 6 \mathrm{~kg} / \mathrm{yr}$

Dilution Factor: 1

(a) Population of Rainier divided by 4, since residents only there for $25 \%$ of the year. 
POPULATION DOSE-CDMMITMENT ESTIMATES AND

AVERAGE INDIVIDUAL DOSE-COMMITMENT HISTOGRAM FOR

TROJAN

Dose Commitments (person-rem) from Liquid Pathways

Total Body GI-LLI Thyroid Bone Liver

Infant

3.9E -07

3.6E-07

$1.8 \mathrm{E}-06$

2.8E -07

4.3E- -07

Child

1. $1 \mathrm{E}-03$

$1.4 \mathrm{E}-03$

2.9E-04

$5.1 \mathrm{E}-03$

$5.7 \mathrm{E}-03$

Teen

Adult

2. $0 \mathrm{E}-03$

2. $9 \mathrm{E}-03$

2.1E-04

3. $1 \mathrm{E}-03$

4. $8 \mathrm{E}-03$

2.1E-02

2. 5E-02

$1.4 \mathrm{E}-03$

1. $8 \mathrm{E}-02$

2. $9 \mathrm{E}-02$

TOTAL

2. $4 \mathrm{E}-02$

3. $0 \mathrm{E}-02$

1.9E-03

2. $6 \mathrm{E}-02$

3. $9 \mathrm{E}-02$

Dose Commitments (person-rem) from Airborne Pathways

Total Body GI-LLI Thyroid Bone Liver Lung

Infant

$1.7 \mathrm{E}-04 \quad 1.6 \mathrm{E}-04 \quad 1.0 \mathrm{E}-03$

2.5E-04

$1.6 \mathrm{E}-04$

2.0E-04

Child

3. $0 \mathrm{E}-03$

2.0E-03

$1.1 \mathrm{E}-02$

$7.7 \mathrm{E}-03$

2.0E -03

2.5E -03

Teen

$1.9 \mathrm{E}-03$

$1.5 \mathrm{E}-03$

$5.5 \mathrm{E}-03$

4. $5 \mathrm{E}-03$

$1.4 \mathrm{E}-03$

2.0E -03

Adult

1.1E-02

8.7E-03

2.5E-02

2. $4 \mathrm{E}-02$

$8.4 \mathrm{E}-03$

$1.0 \mathrm{E}-02$

TOTAL

1.6E-02

1.2E-02

4.2E -02

3. $6 \mathrm{E}-02$

1.2E -02

1.5E-02

Production/Consumption factors:

Produce: $<1$

Milk: <1

Meat: $<1$

FRACTION OF POPULATION RECEIVING AN INDICATED AVERAGE TOTAL-BODY DOSE COMMITMENT FROM AIRBORNE PATHWAYS

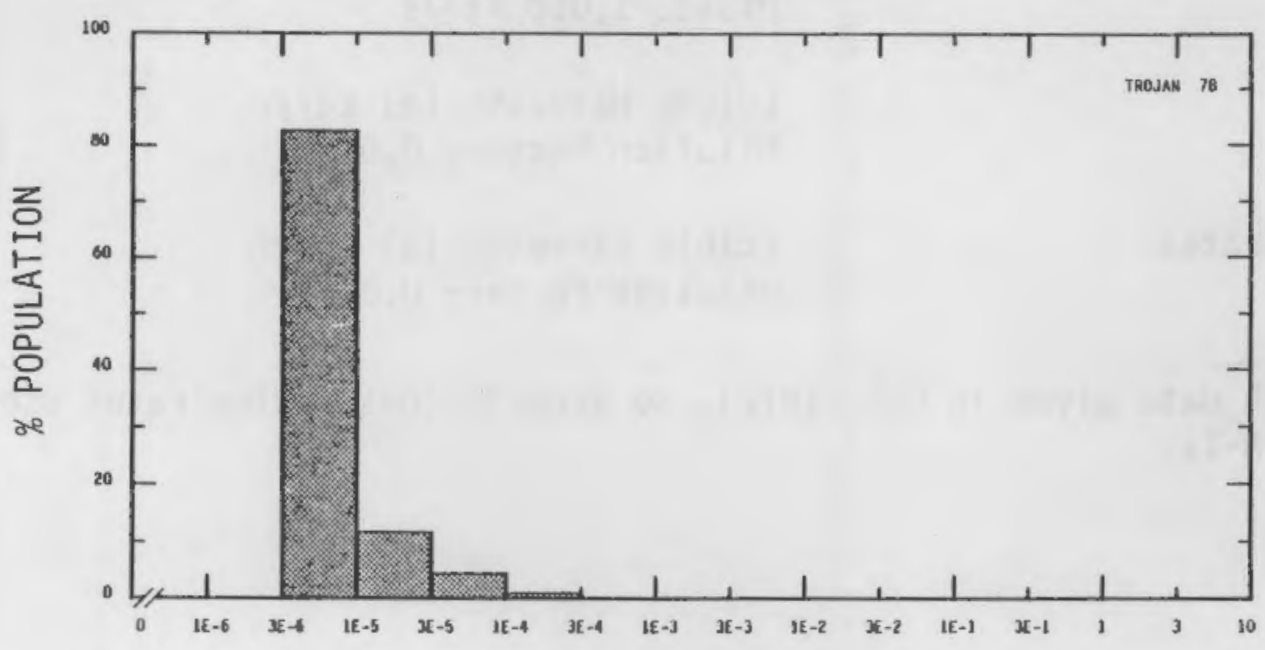

AVG INDIVIDUAL DOSE COMMITMENT - MILLIREM 
Location: N $25.43360 \quad$ W 80.33170

POPULATION DATA

Total Population Within 20-to-80-km Region: 2.1E6

Major Metropolitan Centers Within Region:

Center Population Location

$\begin{array}{llll}\text { Miami SMSA } & 1,600,000 & 48 \mathrm{~km} & \text { NNE }\end{array}$

SITE SPECIFIC DATA - AIRBORNE PATHWAYS

Average Annual State Production

of Crops and Animal Products

In 80-km Radius Circle

Regional Productivity Factor:

Animal Grazing Factor:

Meteorology Period of Record: 1 JAN 73 - 31 DEC 73 Recovery: 98\%

SITE SPECIFIC DATA - WATERBORNE PATHWAYS

Average Dilution Flow from

P1 ant: $1,010 \mathrm{ft} 3 / \mathrm{s}$

Fish:

Edible Harvest: (a) $\mathrm{kg} / \mathrm{yr}$

Dilution Factor: 0.001

Invertebrates:

Edible Harvest: (a) $\mathrm{kg} / \mathrm{yr}$

Dilution Factor: 0.002

(a) No catch data given in DES (1972), so generic consumption rates used (Table A-1). 
POPULATION DOSE-COMMITMENT ESTIMATES AND

AVERAGE INDIVIDUAL DOSE-COMMITMENT HISTOGRAM FOR

TURKEY POINT 3 AND 4

Dose Commitments (person-rem) from Liquid Pathways

Total Body

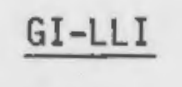

Thyroid

Bone

Liver

Infant

Child

$0.0 \mathrm{E}+00$

$0.0 \mathrm{E}+00$

$0.0 E+00$

$0.0 E+00$

$0.0 \mathrm{E}+00$

Teen

1. $2 \mathrm{E}+00$

2. $8 \mathrm{E}+00$

2. $5 \mathrm{E}+00$

6. $9 \mathrm{E}-01$

$1.1 \mathrm{E}+00$

Adult

9. $3 \mathrm{E}-01$

$6.0 \mathrm{E}+00$

1. $8 \mathrm{E}+00$

4.1E-01

9. $8 \mathrm{E}-01$

$6.7 E+00$

$5.3 \mathrm{E}+01$

1. $2 \mathrm{E}+01$

2. $4 E+00$

$5.9 \mathrm{E}+00$

TOTAL.

$8.8 \mathrm{E}+00$

$6.2 \mathrm{E}+01$

1. $6 \mathrm{E}+01$

3. $5 \mathrm{E}+00$

8. $0 \mathrm{E}+00$

Dose Commitments (person-rem) from Airborne Pathways

Total Body GI-LLI Thyroid Bone Liver Lung

Infant
Child
Teen
Adult
TOTAL

4.7E-03

4. 5E-03

1.9E-01

5. 0 E- 03

$5.1 \mathrm{E}-03$

5. $0 \mathrm{E}-03$

5.2E-02

5.1E-02

1. $1 \mathrm{E}+00$

5.3E-02

5.3E-02

5.7E-02

3.7 $\mathrm{E}-02$

3.7E-02

4. $4 \mathrm{E}-01$

3. $7 \mathrm{E}-02$

3. $8 \mathrm{E}-02$

4. 5E-02

2. $2 \mathrm{E}-01$

2. $3 E-01$

1. $6 \mathrm{E}+00$

2. $2 \mathrm{E}-01$

2. 3E-01

2. 5E-01

3. $2 \mathrm{E}-01$

3. $2 \mathrm{E}-01$

$3.3 E+00$

3. $2 E-01$

3. $2 \mathrm{E}-01$

3. $6 \mathrm{E}-01$

Production/Consumption factors:
Produce: $<1$
Milk: <1
Meat: <1

FRACTION OF POPULATION RECEIVING AN INDICATED AVERAGE TOTAL-BOOY DOSE COMMITMENT FROM AIRBORNE PATHWAYS

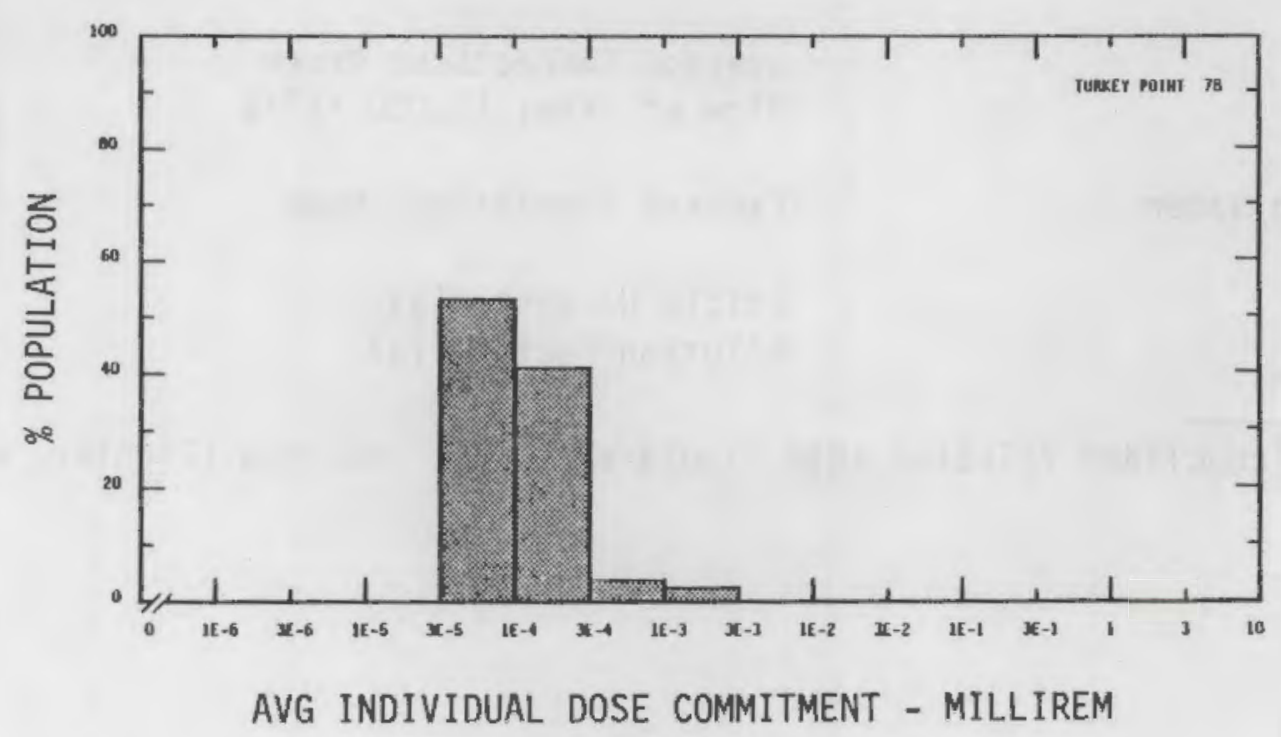



Location:
N $42.7803^{\circ}$
W $72.5158^{\circ}$

POPULATION DATA

Total Population Within 20-to-80-km Region: $1.4 \mathrm{E} 6$

Major Metropolitan Centers Within Region:

\begin{tabular}{lrlll}
\multicolumn{1}{c}{ Center } & Population & \multicolumn{2}{c}{ Location } \\
\cline { 2 - 5 } Pittsfield & 62,000 & $70 \mathrm{~km}$ & SW \\
Springfield SMSA & 640,000 & $72 \mathrm{~km}$ & S \\
Worcester SMSA & 700,000 & $80 \mathrm{~km}$ & SE
\end{tabular}

SITE SPECIFIC DATA - AIRBORNE PATHWAYS

Average Annual State Production

of Crops and Animal Products

In 80-km Radius Circle

Regional Productivity Factor:

Animal Grazing Factor:
Veg: $4.4 E 6$ kilogram

Milk: 7.3E8 liter

Meat: $2.7 \mathrm{E} 7 \mathrm{kilogram}$

1

0.4

Meteorology Period of Record: 1 APR 75 - 31 MAR 76 Recovery: 97\%

SITE SPECIFIC DATA - WATERBORNE PATHWAYS

Average Connecticut River

F1 ow at Site: $10,000 \mathrm{ft}^{3} / \mathrm{s}$

Drinking Water:

Exposed Population: None

Fish:

Edible Harvest: (a)

Dilution Factor: (a)

(a) No radionuclides released into liquid effluents reported (Tichler, et al., 1981 ). 
POPULATION DOSE-COMMITMENT ESTIMATES AND

AVERAGE INDIVIDUAL DOSE-COMMITMENT HISTOGRAM FOR

VERMONT YANKEE

Dose Commitments (person-rem) from Liquid Pathways

Total Body GI-LLI Thyroid Bone

$\begin{array}{llllll}\text { Infant } & 0.0 \mathrm{E}+00 & 0.0 \mathrm{E}+00 & 0.0 \mathrm{E}+00 & 0.0 \mathrm{E}+00 & 0.0 \mathrm{E}+00 \\ \text { Child } & 0.0 \mathrm{E}+00 & 0.0 \mathrm{E}+00 & 0.0 \mathrm{E}+00 & 0.0 \mathrm{E}+00 & 0.0 \mathrm{E}+00 \\ \text { Teen } & 0.0 \mathrm{E}+00 & 0.0 \mathrm{E}+00 & 0.0 \mathrm{E}+00 & 0.0 \mathrm{E}+00 & 0.0 \mathrm{E}+00 \\ \text { Adult } & 0.0 \mathrm{E}+00 & 0.0 \mathrm{E}+00 & 0.0 \mathrm{E}+00 & 0.0 \mathrm{E}+00 & 0.0 \mathrm{E}+00 \\ \text { TOTAL } & 0.0 \mathrm{E}+00 & 0.0 \mathrm{E}+00 & 0.0 \mathrm{E}+00 & 0.0 \mathrm{E}+00 & 0.0 \mathrm{E}+00\end{array}$

Dose Commitments (person-rem) from Airborne Pathways Total Body GI-LLI Thyroid Bone Liver Lung

$\begin{array}{lllllll}\text { Infant } & 3.6 \mathrm{E}-03 & 3.5 \mathrm{E}-03 & 1.3 \mathrm{E}-01 & 3.6 \mathrm{E}-03 & 3.9 \mathrm{E}-03 & 3.7 \mathrm{E}-03 \\ \text { Child } & 4.0 \mathrm{E}-02 & 3.9 \mathrm{E}-02 & 8.8 \mathrm{E}-01 & 3.9 \mathrm{E}-02 & 4.2 \mathrm{E}-02 & 4.3 \mathrm{E}-02 \\ \text { Teen } & 2.9 \mathrm{E}-02 & 2.8 \mathrm{E}-02 & 4.4 \mathrm{E}-01 & 2.8 \mathrm{E}-02 & 3.0 \mathrm{E}-02 & 3.3 \mathrm{E}-02 \\ \text { Adult } & 1.7 \mathrm{E}-01 & 1.7 \mathrm{E}-01 & 1.9 \mathrm{E}+00 & 1.6 \mathrm{E}-01 & 1.7 \mathrm{E}-01 & 1.8 \mathrm{E}-01 \\ \text { TOTAL } & 2.4 \mathrm{E}-01 & 2.4 \mathrm{E}-01 & 3.3 \mathrm{E}+00 & 2.3 \mathrm{E}-01 & 2.5 \mathrm{E}-01 & 2.6 \mathrm{E}-01\end{array}$

Production/Consumption factors:

Produce: <1 Milk: $3.9 \quad$ Meat: <1

FRACTION OF POPULATION RECEIVING AN INDICATED AVERAGE TOTAL-BODY DOSE COMMITMENT FROM AIRBORNE PATHWAYS

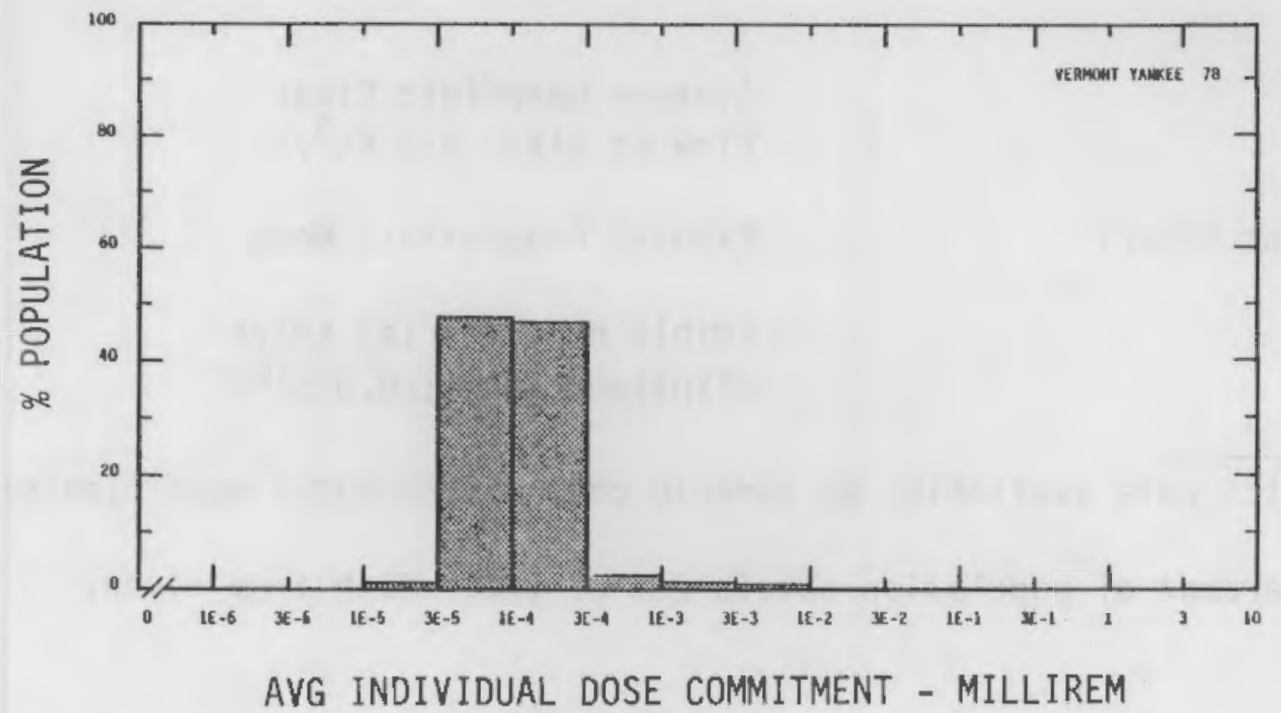


Location: N 42.72810 W 72.92470

POPULATION DATA

Total Population Within 20-to-80-km Region: $1.6 \mathrm{E} 6$

Major Metropolitan Centers Within Region:

\begin{tabular}{lrrrr}
\multicolumn{1}{c}{ Center } & Population & \multicolumn{2}{c}{ Location } \\
\cline { 2 - 5 } Springfield SMSA & 590,000 & & $64 \mathrm{~km}$ & SSE \\
Pittsfield & 58,000 & $34 \mathrm{~km}$ & SW \\
Albany SMSA & 790,000 & $72 \mathrm{~km}$ & W
\end{tabular}

SITE SPECIFIC DATA - AIRBORNE PATHWAYS

Average Annual State Production

of Crops and Animal Products

In 80-km Radius Circle

Regional Productivity Factor:

Animal Grazing Factor:
Veg: $2.0 \mathrm{E} 7 \mathrm{kilogram}$

Milk: 2.6E8 1iter

Meat: $1.6 \mathrm{E} 7 \mathrm{kilogram}$

1

0.5

Meteorology Period of Record: 1 OCT 71 - 30 SEP 72 Recovery: 94\%

SITE SPECIFIC DATA - WATERBORNE PATHWAYS

Average Deerfield River

Flow at Site: $570 \mathrm{ft}^{3} / \mathrm{s}$

Drinking Water:

Exposed Population: None

Fish:

Edible Harvest: (a) $\mathrm{kg} / \mathrm{yr}$ Dilution Factor: 0.025 (b)

(a) No catch data available, so generic consumption rates used (Table A-1).

(b) Ten percent of population obtain $25 \%$ of their fish from river. 
POPULATION DOSE-COMMITMENT ESTIMATES AND AVERAGE INDIVIDUAL DOSE-COMMITMENT HISTOGRAM FOR YANKEE ROWE

Dose Commitments (person-rem) from Liquid Pathways Total Body GI-LLI $\underline{\text { Thyroid Bone Liver }}$

$\begin{array}{llllll}\text { Infant } & 0.0 \mathrm{E}+00 & 0.0 \mathrm{E}+00 & 0.0 \mathrm{E}+00 & 0.0 \mathrm{E}+00 & 0.0 \mathrm{E}+00 \\ \text { Child } & 4.2 \mathrm{E}-02 & 8.6 \mathrm{E}-03 & 5.5 \mathrm{E}-02 & 1.9 \mathrm{E}-01 & 2.0 \mathrm{E}-01 \\ \text { Teen } & 7.1 \mathrm{E}-02 & 7.4 \mathrm{E}-03 & 3.9 \mathrm{E}-02 & 1.2 \mathrm{E}-01 & 1.7 \mathrm{E}-01 \\ \text { Adult } & 7.5 \mathrm{E}-01 & 5.1 \mathrm{E}-02 & 2.6 \mathrm{E}-01 & 6.8 \mathrm{E}-01 & 1.0 \mathrm{E}+00 \\ \text { TOTAL } & 8.6 \mathrm{E}-01 & 6.7 \mathrm{E}-02 & 3.5 \mathrm{E}-01 & 9.9 \mathrm{E}-01 & 1.4 \mathrm{E}+00\end{array}$

Dose Commitments (person-rem) from Airborne Pathways Total Body GI-LLI Thyroid Bone Liver Lung

$\begin{array}{lllllll}\text { Infant } & 1.5 E-01 & 2.6 E-01 & 1.0 E-02 & 9.9 E-02 & 3.1 E-01 & 3.3 E-02 \\ \text { Child } & 1.4 E+00 & 4.6 E-01 & 1.1 E-01 & 8.8 E-01 & 2.1 E+00 & 4.9 E-01 \\ \text { Teen } & 5.3 E-01 & 4.9 E-01 & 7.6 E-02 & 3.6 E-01 & 1.1 E+00 & 4.3 E-01 \\ \text { Adult } & 1.7 E+00 & 2.2 E+00 & 4.5 E-01 & 1.3 E+00 & 3.2 E+00 & 1.9 E+00 \\ \text { TOTAL } & 3.7 E+00 & 3.4 E+00 & 6.5 E-01 & 2.7 E+00 & 6.7 E+00 & 2.9 E+00\end{array}$

Production/Consumption factors:

Produce: <1 Milk: $1.3 \quad$ Meat: <1

FRACTION OF POPULATION RECEIVING AN INDICATED AVERAGE TOTAL-BODY DOSE COMMITMENT FROM AIRBORNE PATHWAYS

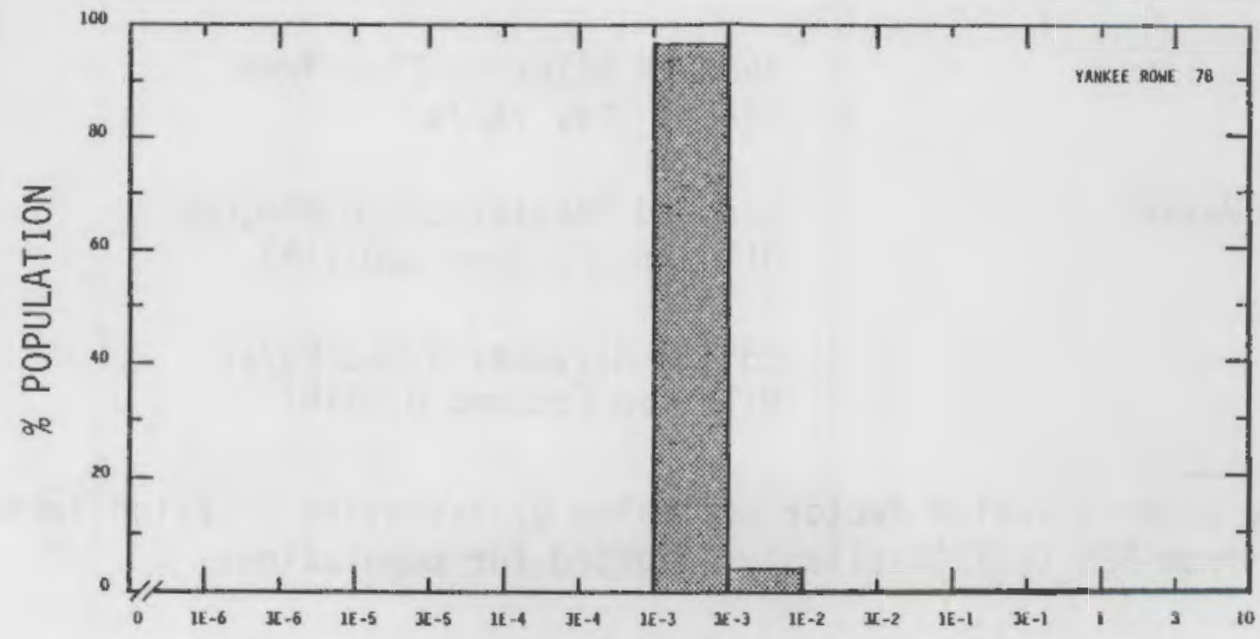

AVG INDIVIDUAL DOSE COMMITMENT - MILLIREM 
Location:

W 87.80610

POPULATION DATA

Total Population Within 20-to-80-km Region: 7.0E6

Major Metropolitan Centers Within Region:

\begin{tabular}{lcccc}
\multicolumn{1}{c}{ Center } & Population & \multicolumn{2}{c}{ Location } \\
\cline { 4 - 5 } & Chicago SMSA (2/3) & $4,700,000$ & $64 \mathrm{~km}$ & SSE \\
Milwaukee SMSA & $1,400,000$ & $64 \mathrm{~km}$ & $\mathrm{~N}$ \\
Kenosha & 80,000 & $14 \mathrm{~km}$ & NNW
\end{tabular}

SITE SPECIFIC DATA - AIRBORNE PATHWAYS

Average Annual State Production

of Crops and Animal Products

In $80-\mathrm{km}$ Radius Circle

Regional Productivity Factor:

Animal Grazing Factor:
Veg: $1.1 \mathrm{~EB}$ kilogram

Milk: $1.8 \mathrm{E} 8$ liter

Meat: $1.9 E 8$ kilogram

0.5

0.5

Meteorology Period of Record: 1 JAN $74-31$ DEC 75 Recovery: 88\%

SITE SPECIFIC DATA - WATERBORNE PATHWAYS

Average Dilution Flow from

Plant: $1291 \mathrm{ft}^{3} / \mathrm{s}$

Drinking Water:

Exposed Population: $6,700,000$

Dilution Factor: $0.037(\mathrm{a})$

Fish:

Edible Harvest: $5.0 \mathrm{E} 6 \mathrm{~kg} / \mathrm{yr}$

Dilution Factor: 0.01 (b)

(a) Drinking water dilution factor estimated by averaging dilution factors derived from FES (1972) suitably weighted for population.

(b) Dilution factor derived from FES (1972). 
POPULATION DDSE-COMMITMENT ESTIMATES AND

AVERAGE INDIVIDUAL DOSE-COMMITMENT HISTOGRAM FOR

ZION 1 ANO 2

Dose Commitments (person-rem) from Liquid Pathways

Total Body

$\underline{\text { GI-LLI }}$

Thyroid

Bone

Liver

Infant

Child

1.6E-01

1. $2 \mathrm{E}-01$

1. $7 \mathrm{E}-01$

2.2E-01

3.2E-01

2. $2 E+00$

1. $4 \mathrm{E}+00$

1.7 +00

3. $6 \mathrm{E}+00$

4. $2 \mathrm{E}+00$

Teen

Adult

1. $3 E+00$

6. $0 \mathrm{E}-01$

$6.2 \mathrm{E}-01$

1. $3 E+00$

2.1E+00

$1.2 \mathrm{E}+01$

$5.2 \mathrm{E}+00$

$5.1 E+00$

8. $8 \mathrm{E}+00$

$1.4 \mathrm{E}+01$

TOTAL

$1.6 \mathrm{E}+01$

$7.3 \mathrm{E}+00$

7. $7 \mathrm{E}+00$

$1.4 E+01$

$2.1 E+01$

Dose Commitments (person-rem) from Airborne Pathways Total Body GI-LLI Thyroid Bone Liver Lung

$\begin{array}{lllllll}\text { Infant } & 9.5 \mathrm{E}-02 & 9.5 \mathrm{E}-02 & 1.2 \mathrm{E}-01 & 9.6 \mathrm{E}-02 & 9.7 \mathrm{E}-02 & 1.0 \mathrm{E}-01 \\ \text { Child } & 1.1 \mathrm{E}+00 & 1.1 \mathrm{E}+00 & 1.2 \mathrm{E}+00 & 1.1 \mathrm{E}+00 & 1.1 \mathrm{E}+00 & 1.2 \mathrm{E}+00 \\ \text { Teen } & 7.8 \mathrm{E}-01 & 7.7 \mathrm{E}-01 & 8.7 \mathrm{E}-01 & 7.8 \mathrm{E}-01 & 7.8 \mathrm{E}-01 & 9.0 \mathrm{E}-01 \\ \text { Adult } & 4.7 \mathrm{E}+00 & 4.7 \mathrm{E}+00 & 5.1 \mathrm{E}+00 & 4.7 \mathrm{E}+00 & 4.7 \mathrm{E}+00 & 5.1 \mathrm{E}+00 \\ \text { TOTAL } & 6.6 \mathrm{E}+00 & 6.6 \mathrm{E}+00 & 7.3 \mathrm{E}+00 & 6.6 \mathrm{E}+00 & 6.7 \mathrm{E}+00 & 7.2 \mathrm{E}+00\end{array}$

Production/Consumption factors:

Produce: $<1 \quad$ Milk: <1 Meat: <1

FRACTION OF POPULATION RECEIVING AN INDICATED AVERAGE

TOTAL-BODY DOSE COMMITMENT FROM AIRBORNE PATHWAYS

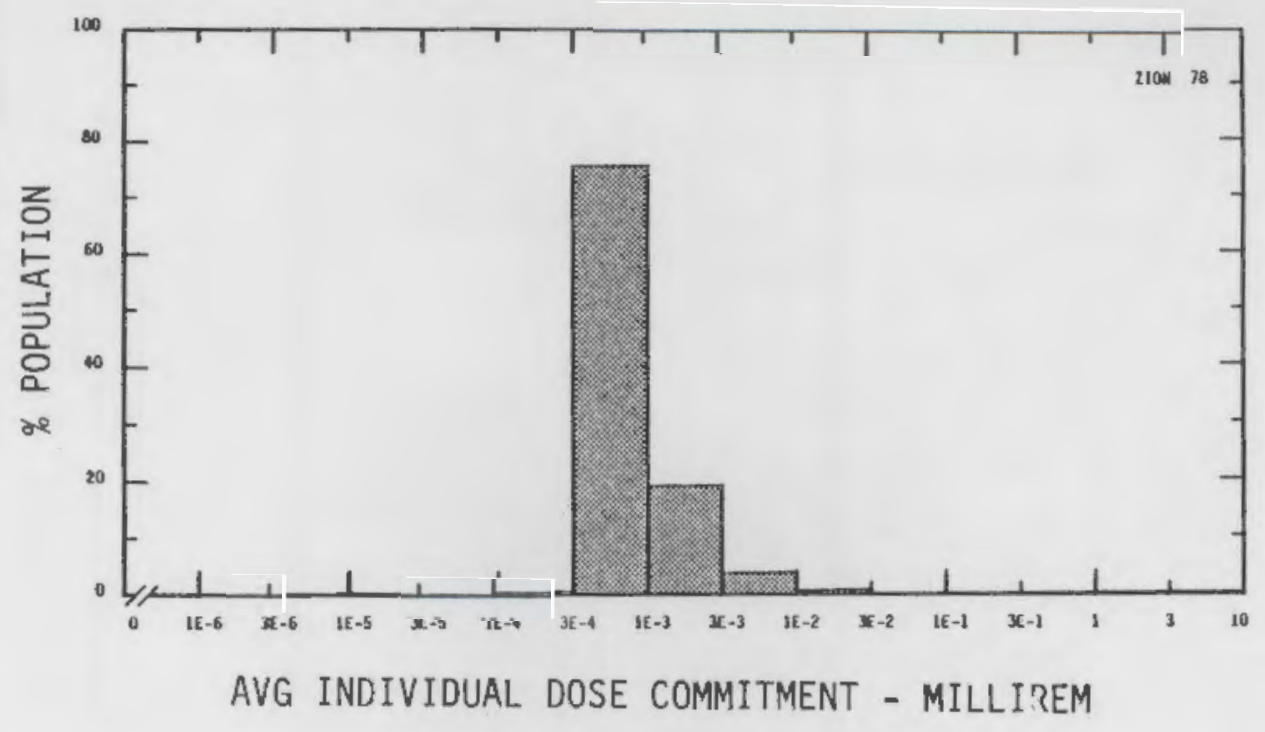





\section{REFERENCES}

Baker, D. A., J. K. Soldat and E. C. Watson. 1977. Population Dose Commitments Due to Radioactive Releases from Nuclear Power Plant Sites in 1975, PNL-2439. Pacific Northwest Laboratory, Richland, WA.

Baker, D. A. 1979. Population Dose Commitments Due to Radioactive Releases from Nuclear Power Plant Sites in 1976, NUREG/CR-1125, PNL2940. U.S. Nuclear Regulatory Commission, Washington, D.C.*

Baker, D. A. 1980. Population Dose Commitments Due to Radioactive Releases from Nuclear Power Plant Sites in 1977, NUREG/CR-1498, PNL3324. U.S. Nuclear Regulatory Commission, Washington, D.C.*

Final Environmental Statement Concerning Proposed Rule-Making Action: Numerical Guides for Design Objectives and Limiting Conditions for Operation to Meet the Criterion "As Low As Practicable" for Radioactive Material in Light-Water-Cooled Nuclear Power Reactor Effluents. 1973. WASH-1258, Vo1. 1. Directorate of Regulatory Standards, U.S. Atomic Energy Commission, Washington, D.C.

Noshkin, V. E., W. L. Robison and F. L. Harrison. 1976. Radiological Dose to Man Through the Marine Pathway from Reactor Operations at Humboldt Bay, California, UCRL-52160. Lawrence Livermore Laboratory, Livermore, CA.

Tichler, J. and C. Benkovitz. 1981. Radioactive Materials Released from Nuclear Power Plants, Annual Report 1978, NUREG/CR-1497, BNL-NUREG51192. U.S. Nuclear Regulatory Commission, Washington, D.C.*

*Available for purchase from the NRC/GPO Sales Program, U.S. Nuclear Regulatory Commission, Washington, D.C. 20555, and the National Technical Information Service, Springfield, VA 22161. 


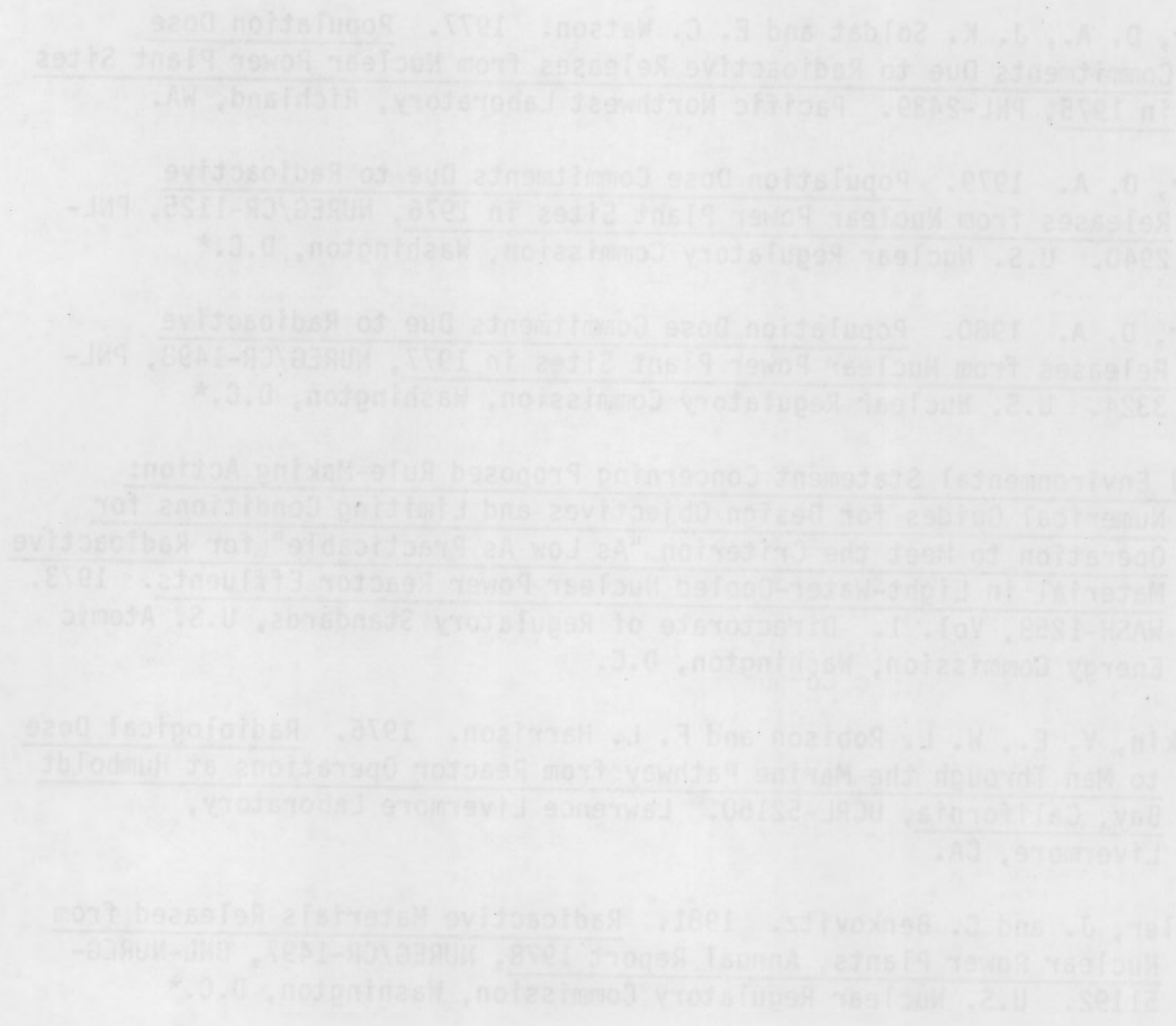

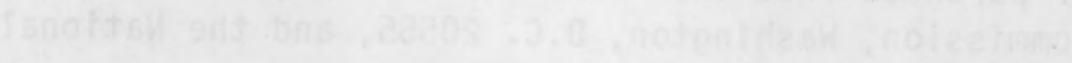




\section{MODELS}

The calculational models used were primarily those given in the Nuclear Regulatory Commission's Regulatory Guide 1.109 (1977). Computer programs were written to use these models to generate population dose commitments for four age groups. The percentages of the population comprising the four age groups were $1.44 \%$, infant; $16.0 \%$, child; $11.7 \%$, teenager; and $70.9 \%$, adult (Population Estimates and Projections, 1975). Where possible, the site-dependent parameters were taken from the environmental statements issued for each reactor (Table 3). The generic parameters used for this study such as consumption rates, occupancy factors and holdup times are given in Table A-1 and A-2 below. It should be noted that generic consumption rates for aquatic foods and inhalation rates are taken from Regulatory Guide 1.109 (1977). Bioaccumulation factors and terrestrial food transfer factors were taken from Regulatory Guide 1.109 (1977). Dose commitment factors for the four age groups were taken from Hoenes and Soldat (1977).

TABLE A-1. Generic Consumption Rates and Occupancy Factors Used for the Study of Average Members of the Population(a)

\begin{tabular}{|c|c|c|c|c|}
\hline Pathway & Infant & Child & Teenager & Adult \\
\hline $\begin{array}{l}\text { Fruits, vegetables and grain } \\
(\mathrm{kg} / \mathrm{yr})\end{array}$ & 0 & 200 & 240 & 190 \\
\hline $\operatorname{Milk}(\ell / y r)$ & 170 & 170 & 200 & 110 \\
\hline Meat and poultry $(\mathrm{kg} / \mathrm{yr})$ & 0 & 37 & 59 & 95 \\
\hline Fish $(\mathrm{kg} / \mathrm{yr})(\mathrm{b})$ & 0 & 2.2 & 5.2 & 6.9 \\
\hline Invertebrates $(\mathrm{kg} / \mathrm{yr})$ & 0 & 0.33 & 0.75 & 1.0 \\
\hline Drinking water $(\ell / y r)$ & $170(c)$ & 260 & 260 & 370 \\
\hline Inhalation $\left(\mathrm{m}^{3} / \mathrm{yr}\right)$ & $1400^{(d)}$ & 3700 & 8000 & 8000 \\
\hline $\begin{array}{l}\text { Air submersion and ground } \\
\text { irradiation occupancy } \\
\text { factor }\end{array}$ & 0.5 & 0.5 & 0.5 & 0.5 \\
\hline
\end{tabular}

(a) Regulatory Guide 1.109 (1977)

(b) Both fresh- and saltwater

(c) Assumed to be equal to milk consumption

(d) Same as for maximum individual 
TABLE A-2. Holdup Times Between Harvest and Consumption of Foods(a)

Food

Fruits, grains and vegetables $\operatorname{Milk}(b)$

Meat(b)

Aquatic foods (fish and invertebrates)

Drinking water
Hol dup Time (days)

14

4

20

7

(a) Regulatory Guide 1.109 (1977)

(b) Value given is time after milking or slaughter. For the portion of the time animals were fed stored, feed, an additional 90 days was added to the holdup time.

\section{RELEASES}

The doses were estimated using the measured releases as reported by the site operators for 1978 (Tichler, et al., 1981).(a) These releases include al radionuclides specified by the NRC to be measured and reported by the operators of all commercial nuclear power plants. Radionuclides given as a combination of parent-daughter isotopes such as $\mathrm{Y} / \mathrm{Sr}-90, \mathrm{Zr} / \mathrm{Nb}-95, \mathrm{Ba} / \mathrm{La}-140$, $\mathrm{I} / \mathrm{Xe}-133$ and $\mathrm{Pr} / \mathrm{Ce}-144$ were divided evently between the parent and daughter.

The radionuclides used in this study, along with their half-lives, are given in Table A-3. Note that the " $D$ " after some of the nuclides indicates that the decay energy of the daughter is included with the parent. Thus, whenever a parent nuclide release is specified, the result of the dose calculation will be as though an additional equilibrium amount of the daughter nuclide is specified. The daughter nuclide itself will be included separately if it can be released independently of the parent and/or if it has a relatively long half-life.

\section{METEOROLOGY}

When more than one set of meteorological (joint frequency) data were available for a site, the one which appeared to be the most reliable was used to generate atmospheric transport factors. Factors were calculated for 16 compass points, and ten radii from 2 to $80 \mathrm{~km}$ (see Table A-4) using the NRC computer program XOQDOQ (Sagendorf 1977).

(a) Very short-1ived isotopes such as $\mathrm{Kr}-90,91,93,94, \mathrm{Xe}-139,140,141$, 143 and $\mathrm{Rb}-88 \mathrm{M}$; those not likely to be produced; and those which were daughters whose decay energies were accounted for in the dose factor for the parent were not included in the dose. 
TABLE A-3. Radionuclides Considered in This Study

\begin{tabular}{|c|c|c|c|c|c|}
\hline No. & Nuclide & $\begin{array}{c}\text { Decay Constant } \\
(1 / \mathrm{sec}) \\
\end{array}$ & No. & Nuclide & $\begin{array}{c}\text { Decay Constant } \\
(1 / \mathrm{sec}) \\
\end{array}$ \\
\hline 1 & $\mathrm{H}-3$ & $1.78 \mathrm{E}-09$ & 43 & $\mathrm{Nb}-97$ & $1.57 \mathrm{E}-04$ \\
\hline 2 & $\mathrm{Be}-10$ & $1.37 \mathrm{E}-14$ & 44 & Mo-99+D & $2.92 \mathrm{E}-06$ \\
\hline 3 & $C-14$ & $3.83 E-12$ & 45 & TC-99M & $3.19 \mathrm{E}-05$ \\
\hline 4 & $\mathrm{~N}-13$ & $1.16 \mathrm{E}-03$ & 46 & $R u-103+D$ & $2.02 E-07$ \\
\hline 5 & $F-18$ & $1.05 \mathrm{E}-04$ & 47 & $R u-106+D$ & 2.17E-08 \\
\hline 6 & $\mathrm{Na}-22$ & $8.44 E-09$ & 48 & $\mathrm{Ag}-110 \mathrm{M}+\mathrm{D}$ & $3.19 E-08$ \\
\hline 7 & $\mathrm{Na}-24$ & $1.28 \mathrm{E}-05$ & 49 & $C d-115 M$ & $1.80 \mathrm{E}-07$ \\
\hline 8 & Ar -41 & $1.05 \mathrm{E}-04$ & 50 & $c d-115$ & $3.60 \mathrm{E}-06$ \\
\hline 9 & Sc -46 & $9.58 \mathrm{E}-08$ & 51 & $S n-125+D$ & $8.31 E-07$ \\
\hline 10 & $\mathrm{Cr}-51$ & $2.89 \mathrm{E}-07$ & 52 & Sb-124 & $1.33 \mathrm{E}-07$ \\
\hline 11 & $M n-54$ & $2.57 E-08$ & 53 & $S b-125+D$ & $8.06 \mathrm{E}-09$ \\
\hline 12 & $M n-56$ & $7.47 \mathrm{E}-05$ & 54 & $\mathrm{Te}-132+\mathrm{D}$ & $2.47 E-06$ \\
\hline 13 & $\mathrm{Fe}-55$ & $8.14 E-09$ & 55 & $T e-133 M+D$ & $2.09 \mathrm{E}-04$ \\
\hline 14 & $\mathrm{Fe}-59$ & $1.80 E-07$ & 56 & $I-131+D$ & $9.97 E-07$ \\
\hline 15 & Co-57 & $2.97 \mathrm{E}-08$ & 57 & I -132 & $8.42 E-05$ \\
\hline 16 & Co-58 & $1.12 \mathrm{E}-07$ & 58 & $I-133+D$ & $9.25 E-06$ \\
\hline 17 & $\mathrm{Co}-60$ & $4.17 \mathrm{E}-09$ & 59 & I -134 & 2.20E-04 \\
\hline 18 & $\mathrm{Ni}-57$ & $5.35 \mathrm{E}-06$ & 60 & $I-135+D$ & $2.92 \mathrm{E}-05$ \\
\hline 19 & $\mathrm{Ni}-63$ & $2.20 E-10$ & 61 & $X e-131 M$ & $6.69 \mathrm{E}-07$ \\
\hline 20 & $\mathrm{Ni}-65$ & $7.64 \mathrm{E}-05$ & 62 & $X e-133 M$ & $3.61 \mathrm{E}-06$ \\
\hline 21 & Cu-64 & $1.52 \mathrm{E}-05$ & 63 & $X e-133$ & $1.52 \mathrm{E}-06$ \\
\hline 22 & $Z n-65$ & $3.31 \mathrm{E}-08$ & 64 & $\mathrm{Xe}-135 \mathrm{M}$ & $7.56 \mathrm{E}-04$ \\
\hline 23 & $Z n-69 M+D$ & 1. $39 \mathrm{E}-05$ & 65 & $X e-135$ & $2.10 \mathrm{E}-05$ \\
\hline 24 & As -76 & 7.32E-06 & 66 & $X e-137$ & $3.01 \mathrm{E}-03$ \\
\hline 25 & $\mathrm{Br}-82$ & $5.44 \mathrm{E}-06$ & 67 & $X e-138+D$ & $8.14 \mathrm{E}-04$ \\
\hline 26 & $\mathrm{Kr}-83 \mathrm{M}$ & $1.04 \mathrm{E}-04$ & 68 & Cs-134 & $1.07 \mathrm{E}-08$ \\
\hline 27 & $\mathrm{Kr}-85 \mathrm{M}$ & 4. $31 \mathrm{E}-05$ & 69 & $C s-136$ & $6.17 \mathrm{E}-07$ \\
\hline 28 & $K r-85$ & $2.05 \mathrm{E}-09$ & 70 & $C s-137+D$ & $7.31 \mathrm{E}-10$ \\
\hline 29 & $\mathrm{Kr}-87$ & $1.52 E-04$ & 71 & Cs -138 & $3.58 \mathrm{E}-04$ \\
\hline 30 & $\mathrm{Kr}-88+\mathrm{D}$ & $6.89 E-05$ & 72 & $C s-139+D$ & $1.24 \mathrm{E}-03$ \\
\hline 31 & $\mathrm{Kr}-89$ & $3.64 \mathrm{E}-03$ & 73 & Ba-139 & 1.39E-04 \\
\hline 32 & $\mathrm{Rb}-88$ & $6.53 E-04$ & 74 & $B a-140+D$ & $6.28 \mathrm{E}-07$ \\
\hline 33 & $R b-89+D$ & $7.61 \mathrm{E}-04$ & 75 & La-140 & $4.78 \mathrm{E}-06$ \\
\hline 34 & $S r-89+D$ & $1.59 \mathrm{E}-07$ & 76 & La-141 & $4.97 \mathrm{E}-05$ \\
\hline 35 & $S r-90+D$ & $7.58 \mathrm{E}-10$ & 77 & $\mathrm{Ce}-141$ & $2.47 E-07$ \\
\hline 36 & $S r-91+D$ & $2.03 \mathrm{E}-05$ & 78 & Ce-144+D & $2.83 E-08$ \\
\hline 37 & $\mathrm{Sr}-92+\mathrm{D}$ & $7.11 \mathrm{E}-05$ & 79 & Eu-152 & $1.69 E-09$ \\
\hline 38 & $Y-90$ & $3.01 \mathrm{E}-06$ & 80 & Eu-154 & $2.55 E-09$ \\
\hline 39 & $Y-91 M+D$ & 2. $32 E-04$ & 81 & $W-187$ & $8.06 \mathrm{E}-06$ \\
\hline 40 & $Z r-95+D$ & $1.22 \mathrm{E}-07$ & 82 & $T h-232+D$ & $1.57 \mathrm{E}-18$ \\
\hline 41 & $Z r-97+D$ & $1.14 \mathrm{E}-05$ & 83 & Np-239 & $3.42 E-06$ \\
\hline 42 & $\mathrm{Nb}-95$ & $2.29 \mathrm{E}-07$ & & & \\
\hline
\end{tabular}


TABLE A-4. Radius Intervals and Midpoints for Airborne Dose Calculations ( $\mathrm{km})$

$\begin{array}{cc}\text { Interval } & \text { Midpoint } \\ 2-3 & \\ 3-4 & 2.5 \\ 4-6 & 3.5 \\ 6-9 & 5 \\ 9-14 & 7.5 \\ 14-20 & 11.5 \\ 20-30 & 17 \\ 30-40 & 25 \\ 40-60 & 35 \\ 60-80 & 50 \\ & 70\end{array}$

The XOQDOQ program generates four sets of atmospheric transport factors:

- average annual atmospheric dilution factors which are not corrected for cloud depletion or radioactive decay

- dilution factors which are only corrected for decay assuming a 2.26-day half-life

- dilution factors which are corrected for depletion and for decay assuming an 8-day half-life

- relative deposition per unit area.

These factors were used to estimate the dose from airborne releases using methods similar to the NRC GASPAR program (Eckerman 1976). Except for the two additional sites, the transport factors used this year were the same as those used for the previous estimates. The assumptions used in the calculation of these transport factors were as follows:

- 50-m source height with no correction for plume rise or building wake effects

- semi-infinite cloud model with sector-average, Gaussian-plume dispersion

- no correction for terrain height variation.

Since information about height and locations at each site for the releases given in Tichler, et al., (1981) was unavailable, a single generic height of $50 \mathrm{~m}$ was used at each site for the release point. Because the heights and locations of releases are uncertain, estimates of dose to persons 
living within $2 \mathrm{~km}$ of the site could be in serious error; only persons living between 2 to $80 \mathrm{~km}$ from the site were included in the dose estimates.

\section{POPULATION}

The population distribution within 2 to $80 \mathrm{~km}$ around each site was determined by using a computer program and data base derived from the 1970 census. The program and data base were developed by the 0epartment of Commerce and subsequently adopted by the Environmental Protection Agency (EPA) for use with population exposure problems (Athey, Tell and Janis 1974, Hil1 1977).

The population date base used was an edited and compressed version of the 1970 Census Bureau's Master Enumeration District List with Coordinates. It contains housing and population counts for each census enumeration district and the geographic coordinates of the population centroid for each district. Using a modified version of the EPA program and the data base, the population in each of the 160 segments around each site was estimated from a distance of 2 to $80 \mathrm{~km}$. The populations for 1978 were estimated using the "net increase" factors by state over the population values for 1970 as given in Statistical Abstract of the United States, 1979 (Table 11).

\section{FOOD PRODUCTION VERSUS FOOD CONSUMPTIDN}

The total food production for the region within $80 \mathrm{~km}$ around each site was the product of the NRC state-wide productivity figure for each state and a site productivity factor. At some sites this total production may be more or less than the total consumption; i.e., population times average individual consumption (see Table A-1 for generic consumption rates). When production was more than consumption for a site, it was assumed that all persons in the 2-to-80-km region ate contaminated food; when production was less than consumption, it was assumed that dilution would occur because uncontaminated food would be shipped into the area from outside. Thus, the calculated doses for a particular food type were reduced in proportion to the ratio of production $\div$ consumption (production/consumption $<1$ ).

The dose to persons outside the 80-km limit from food shipped out of the region, in the case of production being greater than consumption, is not included in this report because it is concerned only with the dose within the $80-\mathrm{km}$ radius. These production/consumption factors are given as footnotes to the tables showing airborne dose commitment in the Site Summary Section for reference.

\section{DRINKING WATER}

The population between 2 and $80 \mathrm{~km}$ of each plant site exposed to drinking water contaminted with released radionuclides was generally obtained 
from the environmental statement (ES) for the plant. For all sites located on salt water, it was assumed that no dose was received from drinking water. The consumption rates used are given in Table A-1 for drinking water.

The radionuclide concentration in the drinking water consumed by a population downstream from a site was usually estimated assuming $100 \%$ mixing of the plant effluent with the river. For lakes, an overall dilution factor was estimated from dilution factors given in the ES for each population center along the shore (within $80 \mathrm{~km}$ ) which consume the contaminted lake water. These individual factors were weighted by population and averaged to obtain an effective dilution factor for the total population exposed to contaminated drinking water.

\section{AQUATIC FOOD}

Wherever possible, the fish-catch data from the plant ES was used to estimate aquatic food consumption rates for the population in the region. When this data was not found in the ES or was considered unrealistic, the generic values of Table A-1 were used.

The average radionuclide concentration of the waters in which this food was harvested was estimated assuming an additional dilution over the effluent flow from the reactor. For rivers, it was assumed that the fish were caught in waters in which the plant effluent was completely diluted. For lakes, an additional factor as given in the ES was used; when none was given in the ES, a generic value of 0.01 was used. For ocean and bay sites, a generic value of 0.001 and 0.002 was used for fish and invertebrates, respectively, if the ES yielded no values for these parameters. Invertebrates were not assumed to be caught in sufficient quantity at freshwater sites (river and 1ake) to affect the population dose and therefore were not included in the dose calculation. Any exceptions to these general guidelines are explained in the footnotes to the individual site summaries. 
APPENDIX REFERENCES

Athey, T. W., R. A. Tell and D. E. Janis. 1974. The Use of an Automated Population Data Base in Population Exposure Calculation, CONF 741018. Proceedings of Symposium on Population Exposures. NTIS, Springfield, VA.

Eckerman, K. 1976. GASPAR DOSE CODE (Computer Listing). U.S. Nuclear Regulatory Commission, Washington, D.C.

Hi11, E. R. 1977. Calculation of Population Distribution Using a Computer Data Base, BNWL-2395. Pacific Northwest Laboratory, Richland, WA.

Hoenes, G. R., and J. K. Soldat. 1977. Age-Specific Radiation Dose Commitment Factors for a One-Year Chronic Intake, NUREG-0172. U.S. Nuclear Regulatory Commission, Washington, D.C. ${ }^{*}$

Population Estimates and Projections, Current Population Reports. 1975. Series P-25, No. 541. U.S. Department of Commerce, Social and Economic Statistics Administration, Bureau of the Census.

Regulatory Guide 1.109. 1977. Calculation of Annual Doses to Man from Routine Releases of Reactor Effluents for the Purpose of Evaluating Compliance with 10 CFR Part 50, Appendix I. Revision 1. U.S. Nuclear Regulatory Commission, Washington, D.C.

Sagendorf, J. F., and J. T. Go11. 1977. XOQDOQ--Program for the Meteorological Evaluation of Routine Effluent Releases at Nulcear Power Stations, NUREG-0324. U.S. Nuclear Regulatory Commission, Washington, D.C. $\star \star$

Statistical Abstract of the United States: 1979. 100th edition. U.S. Department of Commerce, Bureau of the Census, Washington, D.C.

Tichler, J. and C. Benkovitz. 1981. Radioactive Materials Released from Nuclear Power Plants, Annual Report 1978, NUREG-CR-1497, BNL-NUREG51192. U.S. Nuctear Regulatory Commission, Washington, D.C.*

*Available for purchase from the NRC/GPO Sales Program, U.S. Nuclear Regulatory Commission, Washington, D.C. 20555, and the National Technical Information Service, Springfield, VA 22161.

**Available free upon written request to the Division of Technical Information and Document Control, U.S. Nuclear Regulatory Commission, Washington, D.C. 20555. 



\section{DISTRIBUTION}

No. of

Copies

1 AA Churm

DOE Patent Division

9800 S. Cass Avenue

Argonne, IL 60439

2 DOE Technical Information Center

Oak Ridge, TN 37830

30 MR Beebe

office of Management \& Program Analysis

Nuclear Regulatory Commission

Washington, DC 20555

50 Pacific Northwest Laboratory

P.O. Box 999

Richland, WA 99352

OA Baker (39)

RA Peloquin

JD Schwab

BE Vaughan

EC Watson

Publishing Coordinator (2)

Technical Information (5) 



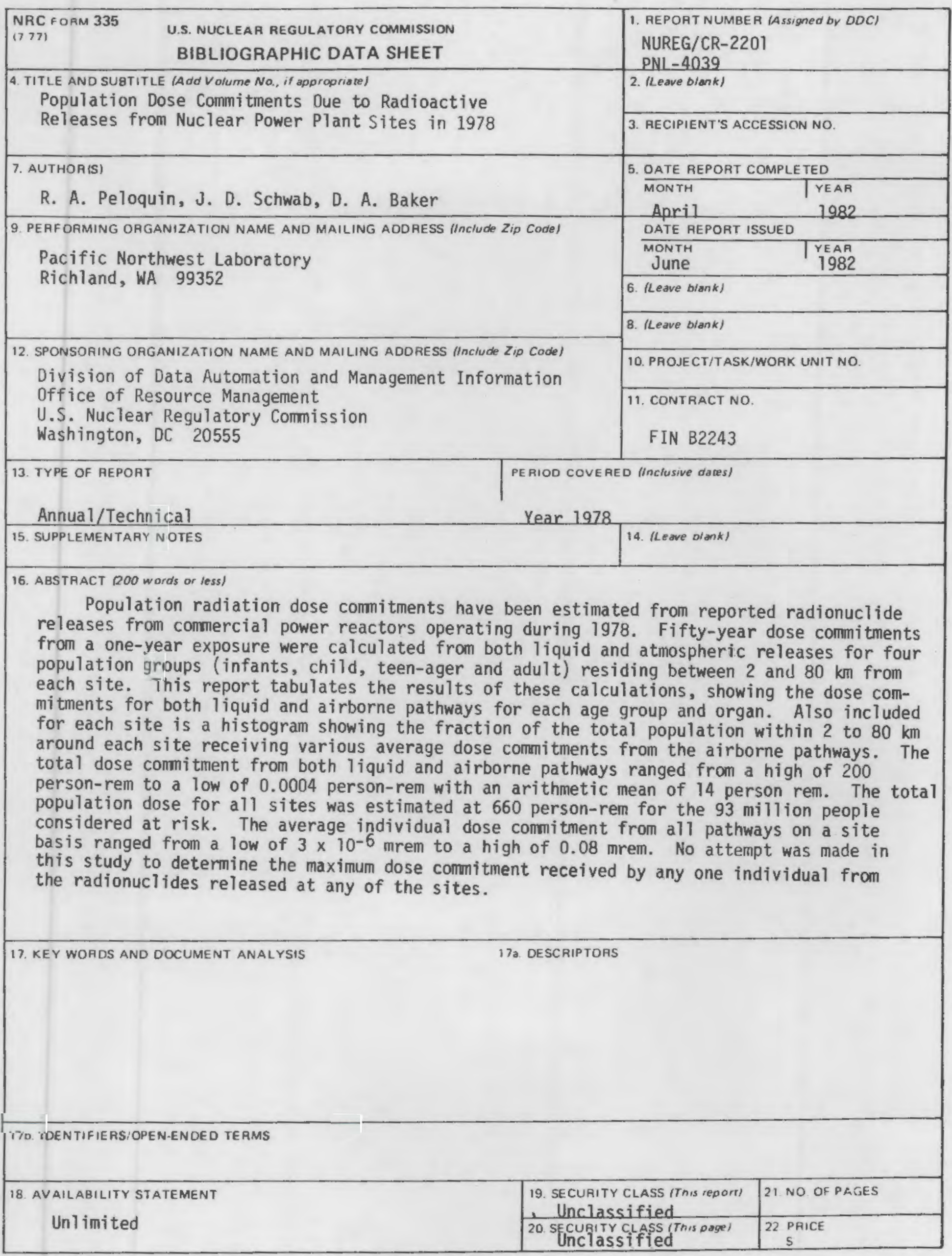




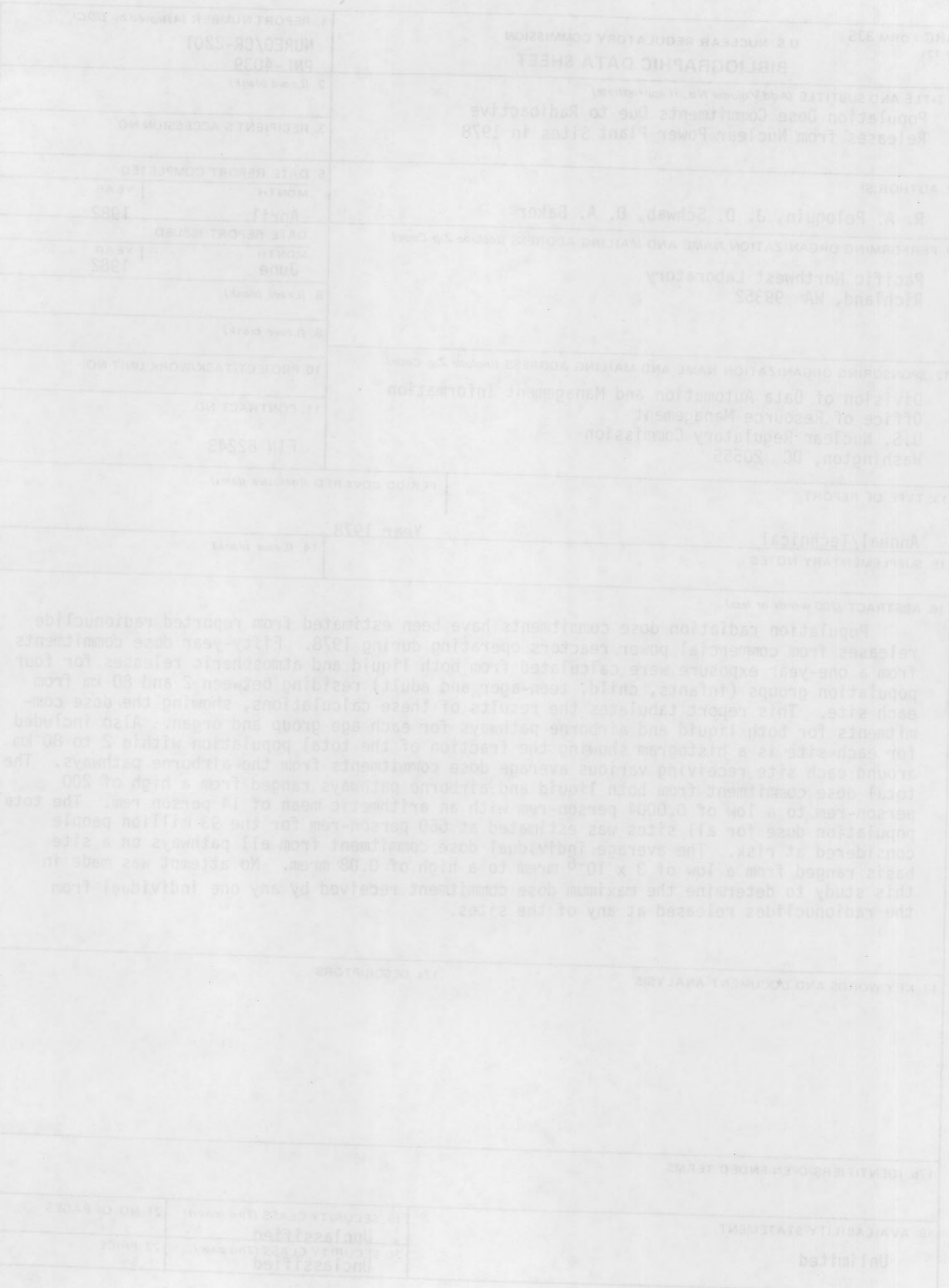

